\title{
8-Iodonaphtalene-1-carbaldehyde: a Versatile Building Block for Diversity-Oriented Synthesis
}

\author{
Lidia Herrera, ${ }^{\dagger}$ Pablo Barrio,,${ }^{\dagger}$ Ignacio Ibáñez, ${ }^{\dagger}$ Raquel Román, ${ }^{\ddagger}$ \\ Natalia Mateu, ${ }^{\S}$ Santos Fustero* $*$ \\ †Departamento de Química Orgánica, Universidad de Valencia, E-46100 Burjassot, Spain \\ ${ }^{\ddagger}$ Laboratorio de Moléculas Orgánicas, Centro de Investigación Príncipe Felipe, E-46012 \\ Valencia, Spain. \\ ${ }^{\S}$ Department of Chemistry, University of Cambridge, Lensfield Road, Cambridge, CB2 IEW, \\ $U K$ \\ santos.fustero@uv.es; pablo.barrio@uv.es
}

\section{SUPPORTING INFORMATION}

\section{Table of contents:}

General methods.......................................... 2

Experimental procedures and characterizations...................S2

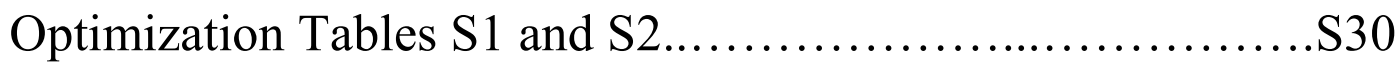

Computational analysis: Principal Moment of Inertia (PMI) .. S31

${ }^{1} \mathrm{H},{ }^{13} \mathrm{C}$ and ${ }^{19} \mathrm{~F}$ Spectra of all new compounds................ S53

HPLC chromatograms of final products...................S171 


\section{General methods:}

Reactions were carried out under a nitrogen atmosphere unless otherwise indicated. The solvents were purified prior to use: THF and PhMe were distilled from sodium/benzophenone; $\mathrm{CH}_{2} \mathrm{Cl}_{2}$ was distilled from calcium hydride; anhydrous 1,4-dioxane, $\mathrm{CHCl}_{3}$ and $\mathrm{MeOH}$ were purchased from Aldrich and used as received. All reagents were obtained from commercial suppliers and used without further purification. The reactions were monitored with the aid of thin-layer chromatography (TLC) on $0.25 \mathrm{~mm}$ E. Merck pre-coated silica gel plates. Visualization was carried out with UV light and aqueous ceric ammonium molybdate solution or vanilla stain. Flash column chromatography was performed with the indicated solvents on silica gel 60 (particle size 0.040-0.063 mm). Melting points were measured on a Büchi Melting Point P-450 apparatus and are uncorrected. Optical rotations were measured on a Jasco P-1020 polarimeter. ${ }^{1} \mathrm{H},{ }^{19} \mathrm{~F}$ and ${ }^{13} \mathrm{C}$ NMR spectra were recorded on a $300 \mathrm{MHz}$ Bruker Advance AC300 spectrometer. Chemical shifts are given in ppm $(\delta)$, with reference to the residual proton resonance of the solvents. Coupling constants $(J)$ are given in Hertz $(\mathrm{Hz})$. The letters $\mathrm{m}, \mathrm{s}, \mathrm{d}, \mathrm{t}$, and $\mathrm{q}$ stand for multiplet, singlet, doublet, triplet and quartet, respectively. The letters br indicate that the signal is broad. High-resolution mass spectra were carried out on VG Autospec (VG Analytical, Micromass Instruments) by the Universidad de Valencia Mass Spectrometry Service. Microwave reactions were carried out in a $2-5 \mathrm{~mL}$ or $15 \mathrm{~mL}$ sealed vial with a Biotage InitiatorTM 2.0 microwave synthesizer.

\section{Experimental procedures and characterizations:}

\section{Synthesis of N-tert-Butanesulfinyl Imines I.}

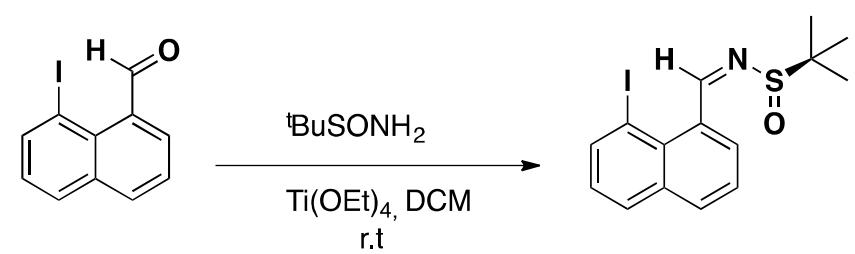

A solution of 8-iodonaphthalene-1-carbaldehyde 1 (1.0 equiv) and $\mathrm{Ti}(\mathrm{OEt})_{4}$ (5.0 equiv) in dichloromethane $(0.1 \mathrm{M})$ was stirred for 5 minutes. $(R)$ - $N$-tert-Butanesulfinyl amine was added to the resulting solution and the mixture was stirred at room temperature for 20 hours. Then, saturated aqueous $\mathrm{NaHCO}_{3}$ was added until white titanium salts precipitated. The suspension was filtered through a short pad of Celite ${ }^{\circledR}$ washing with small portions of ethyl acetate. The filtrate was extracted with ethyl acetate and the combined organic layers were washed with 
brine, dried over anhydrous $\mathrm{Na}_{2} \mathrm{SO}_{4}$, concentrated under vacuum and purified by means of flash chromatography on silica gel employing mixtures of $n$-hexane: ethyl acetate as eluents. ${ }^{1}$

$\left(R_{S}\right)-(E)-N-[(8-I o d o n a p h t h a l e n-1-y l)$ methylene]-2-methylpropane-2-sulfinamide (I)<smiles>CCCCOS(=O)(=O)c1cccc2cccc(I)c12</smiles>

Compound I was synthesized following the procedure for the condensation with Ellman's auxiliary described above starting from $\mathbf{1}^{2}(500 \mathrm{mg}, 1.77 \mathrm{mmol})$ affording $\mathbf{I}$ as a colorless oil (614 mg, $90 \%$ yield). $[\alpha]^{25}=-240.5\left(c\right.$ 1.0, $\left.\mathrm{CHCl}_{3}\right) .{ }^{1} \mathrm{H} \mathrm{NMR}\left(300 \mathrm{MHz}, \mathrm{CDCl}_{3}\right)$ : 1.35 (s, 9H), 7.40 (dd, $J=7.4,8.1 \mathrm{~Hz}, 1 \mathrm{H}), 7.53$ (dd, $J=11.5,3.9 \mathrm{~Hz}, 1 \mathrm{H}), 7.87$ (dd, $J=8.2$, $1.2 \mathrm{~Hz}, 2 \mathrm{H}), 7.96-7.89(\mathrm{~m}, 2 \mathrm{H}), 10.00(\mathrm{~s}, 1 \mathrm{H}) .{ }^{13} \mathrm{C} \mathrm{NMR}\left(75.5 \mathrm{MHz}, \mathrm{CDCl}_{3}\right): 23.0\left(3 \mathrm{xCH}_{3}\right)$, $58.6(\mathrm{C}), 90.1(\mathrm{C}), 125.7(\mathrm{CH}), 127.3(\mathrm{CH}), 129.7(\mathrm{CH}), 130.1(\mathrm{CH}), 133.0(\mathrm{CH}), 133.5(2 \times \mathrm{C})$, $135.5(2 \times C), 141.6(\mathrm{CH}), 164.1(\mathrm{CH})$. HRMS (EI): $\mathrm{m} / \mathrm{z}$ calcd for $\mathrm{C}_{15} \mathrm{H}_{16} \mathrm{INOS}: 386.0070[M+$ $\mathrm{H}^{+}$]; found: 386.0072 .

Diastereoselective addition of the Ruppert Prakash reagent: $\mathrm{CF}_{3} \mathrm{TMS}$ was slowly added to a mixture of imine $\mathbf{I}$ (1.0 equiv) and tetrabutylammonium acetate (1.2 equiv) in THF ( $0.1 \mathrm{M})$ at $-55^{\circ} \mathrm{C}$. The reaction mixture was then stirred at $-55^{\circ} \mathrm{C}$ until TLC revealed the disappearance of the starting material. Next, saturated $\mathrm{NH}_{4} \mathrm{Cl}$ solution was added at this temperature, and the reaction mixture was warmed to room temperature. The quenched reaction mixture was extracted three times with ethyl acetate and the combined organic layers were dried over anhydrous $\mathrm{Na}_{2} \mathrm{SO}_{4}$. Solvents were removed under reduced pressure and the crude mixture was purified by flash chromatography on silica gel using mixtures of $n$-hexane: ethyl acetate as eluents to afford compounds $\mathbf{2 a}$.

$\left(R_{S}\right)$-2-Methyl-N-I(S)-2,2,2-trifluoro-1-(8-iodonaphthalen-1-yl)ethyl]propane-2-sulfinamide (2a)<smiles>CCOS(=O)(=O)NC(c1cccc2cccc(I)c12)C(F)(F)F</smiles>

Compound 2a was synthesized following the procedure for the trifluoromethylation described above starting from $\mathbf{I}(500 \mathrm{mg}, 1.3 \mathrm{mmol})$ affording $2 \mathbf{a}$ as a yellowish oil (260 mg, $44 \%$ yield). $[\alpha]^{25}=-133.8\left(c 1.0, \mathrm{CHCl}_{3}\right) .{ }^{1} \mathrm{H} \mathrm{NMR}\left(300 \mathrm{MHz}, \mathrm{CDCl}_{3}\right)$ :

\footnotetext{
${ }^{1}$ Fustero, S.; Ibañez, I.; Barrio, P.; Maestro, M. A.; Catalán S. Org. Lett. 2013, 15, 832.

${ }^{2}$ Bailey. R. J.; Card. P.; Shechter. H. J. Am. Chem. Soc. 1983, 105, 6096.
} 
$1.26(\mathrm{~s}, 9 \mathrm{H}), 3.89$ (d, $J=6.7 \mathrm{~Hz}, 1 \mathrm{H}), 7.09$ (dd, $J=7.3,1.1 \mathrm{~Hz}, 1 \mathrm{H}), 7.54$ (dd, $J=7.7,0.9 \mathrm{~Hz}$, 1H), 7.80 (ddd, $J=7.0,3.5,2.4 \mathrm{~Hz}, 1 \mathrm{H}), 7.86(\mathrm{~m}, 2 \mathrm{H}), 8.37$ (dd, $J=7.4,1.3 \mathrm{~Hz}, 1 \mathrm{H}) .{ }^{19} \mathrm{~F}$ NMR $\left(282.4 \mathrm{MHz}, \mathrm{CDCl}_{3}\right) \delta-72.53(\mathrm{~d}, J=7.3 \mathrm{~Hz}) .{ }^{13} \mathrm{C} \mathrm{NMR}\left(75.5 \mathrm{MHz}, \mathrm{CDCl}_{3}\right): 22.5\left(3 \mathrm{xCH}_{3}\right)$, $53.9\left(\mathrm{q},{ }^{2} J_{\mathrm{CF}}=30.8 \mathrm{~Hz}, \mathrm{CH}\right), 57.1(\mathrm{C}), 87.8(\mathrm{C}), 119.3(\mathrm{C}), 125.5(\mathrm{CH}), 126.7\left(\mathrm{q},{ }^{1} J_{\mathrm{CF}}=281.4\right.$ $\left.\mathrm{Hz}, \mathrm{CF}_{3}\right), 127.0(\mathrm{CH}), 128.5(\mathrm{CH}), 131.0(\mathrm{CH}), 131.2(\mathrm{C}), 131.4(\mathrm{C}), 132.1(\mathrm{CH}), 136.1(\mathrm{C})$, 144.7 (CH). HRMS (EI): m/z calcd for $\mathrm{C}_{16} \mathrm{H}_{17} \mathrm{NNOSF}_{3}$ : $456.0100\left[M+\mathrm{H}^{+}\right]$; found: 456.0115 .

$\underline{A z a-R e f o r m a t s k y ~ r e a c t i o n: ~ t h e ~ r e q u i r e d ~ R e f o r m a t s k y ~ r e a g e n t ~ w a s ~ p r e p a r e d ~ a s ~ f o l l o w s: ~ t o ~ a ~}$ suspension of activated $\mathrm{Zn}\left(200 \mathrm{mg}, 1.3\right.$ equiv) in refluxing $\mathrm{Et}_{2} \mathrm{O}(5 \mathrm{~mL})$ ethyl bromoacetate $(0.30 \mathrm{~mL})$ was added dropwise. After refluxing the reaction mixture for $1 \mathrm{~h}$ a light green solution was obtained. ${ }^{3}$ This solution was titrated with iodine and its concentration was found to be around 0.4 M. $0.42 \mathrm{~mL}$ of this solution $\left(0.17 \mathrm{mmol}, 1.7\right.$ equiv) were added dropwise at $0{ }^{\circ} \mathrm{C}$ to a solution of $\mathbf{I}(31 \mathrm{mg}, 0.1 \mathrm{mmol})$ in dry THF $(1 \mathrm{~mL})$. After stirring for $15 \mathrm{~min}$ at this temperature the reaction was allowed to reach room temperature, and stirred for another hour. The reaction was quenched with saturated $\mathrm{NH}_{4} \mathrm{Cl}_{\text {(aq.) }}(1 \mathrm{~mL})$ and extracted with AcOEt $(3 \mathrm{x} 1$ $\mathrm{mL}$ ). Flash chromatography afforded $\mathbf{2} \mathbf{b}$ as a colorless oil.

\section{Ethyl (3S)-3-[(RS)(tert-butylsulfinyl)amino)-3-(8-iodonaphthalen-1-yl]propanoate (2b)}

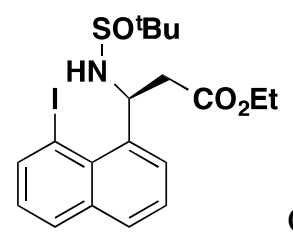

Compound 2b was synthesized following the procedure described above starting from I (500 mg, $1.3 \mathrm{mmol}$ ) affording $\mathbf{2 b}$ as a yellowish oil (430 mg, $70 \%$ yield). $[\alpha]^{25}$ $=+2.7\left(c=1.0\right.$ in $\left.\mathrm{CHCl}_{3}\right) .{ }^{1} \mathrm{H}$ NMR $\left(300 \mathrm{MHz}, \mathrm{CDCl}_{3}\right): 1.13(\mathrm{t}, J=7.1 \mathrm{~Hz}, 3 \mathrm{H}), 1.24(\mathrm{~s}, 9 \mathrm{H})$, $2.91(\mathrm{dd}, J=7.8,5.9 \mathrm{~Hz}, 1 \mathrm{H}), 3.17(\mathrm{dd}, J=15.9,4.3 \mathrm{~Hz}, 1 \mathrm{H}), 4.05$ (q, $J=7.1 \mathrm{~Hz}, 2 \mathrm{H}), 5.01$ (d, $J=5.1 \mathrm{~Hz}, 1 \mathrm{H}), 7.04(\mathrm{dd}, J=8.0,7.4 \mathrm{~Hz}, 1 \mathrm{H}), 7.14(\mathrm{dd}, J=7.8,7.4 \mathrm{~Hz}, 1 \mathrm{H}), 7.48$ (dd, $J=7.7$, $1.5 \mathrm{~Hz}, 1 \mathrm{H}), 7.75$ (dd, $J=8.1,1.3 \mathrm{~Hz}, 1 \mathrm{H}), 7.83(\mathrm{dd}, J=8.2,1.5 \mathrm{~Hz}, 2 \mathrm{H}), 8.31$ (dd, $J=7.4,1.3$ $\mathrm{Hz}, 1 \mathrm{H}) .{ }^{13} \mathrm{C}$ NMR $\left(75.5 \mathrm{MHz}, \mathrm{CDCl}_{3}\right): 14.2\left(\mathrm{CH}_{3}\right), 22.8\left(3 \mathrm{xCH}_{3}\right), 43.2\left(\mathrm{CH}_{2}\right), 49.7(\mathrm{CH}), 55.9$ (C), $60.9\left(\mathrm{CH}_{2}\right), 87.6(\mathrm{C}), 125.5(\mathrm{CH}), 126.4(\mathrm{CH}), 127.9(\mathrm{CH}), 130.2(\mathrm{CH}), 130.8(\mathrm{CH}), 131.2$ (C), $136.1(\mathrm{C}), 137.3(\mathrm{C}), 143.7(\mathrm{CH}), 171.5(\mathrm{C})$. HRMS (EI): $\mathrm{m} / \mathrm{z}$ calcd for $\mathrm{C}_{19} \mathrm{H}_{24} \mathrm{INO}_{3} \mathrm{~S}$ : $474.0591\left[M+\mathrm{H}^{+}\right]$; found: 474.0594 .

Reaction of Compound I with Allylzinc Bromide: A 1M solution of allylzinc bromide in THF was prepared by stirring $0.4 \mathrm{~mL}$ of allyl bromide and $1.0 \mathrm{~g}$ of activated $\mathrm{Zn}$ in $2.5 \mathrm{~mL}$ of anhydrous THF at $50^{\circ} \mathrm{C} .2$ equivalents of this freshly prepared solution were then added to a solution of substrate $\mathbf{I}$ in dry $\mathrm{THF}$ at $-40{ }^{\circ} \mathrm{C}$. After stirring $10 \mathrm{~min}$ at $0{ }^{\circ} \mathrm{C}$ the reaction mixture

\footnotetext{
${ }^{3}$ Greszler, S. N.; Johnson, J. S. Angew. Chem. Int. Ed. 2009, 48, 3689.
} 
was quenched with saturated $\mathrm{NH}_{4} \mathrm{Cl}_{(\mathrm{aq} .)}$ and extracted three times with AcOEt. Flash chromatography afforded compound $\mathbf{2 c}$ as a colorless oil.

N-( $\left.\left.R_{S}\right)-1-[(S)-8-I o d o n a p h t h a l e n-1-y l) b u t-3-e n-1-y l\right]-2-m e t h y l p r o p a n e-2-s u l f i n a m i d e$

(2c)<smiles>C=CCC(NS(C)(=O)=O)c1cccc2cccc(I)c12</smiles>

Compound $2 \mathbf{c}$ was synthesized following the procedure described above starting from I (500 mg, $1.3 \mathrm{mmol}$ ) affording $\mathbf{2 c}$ as a colorless oil (333 $\mathrm{mg}, 60 \%$ yield). $[\alpha]_{\mathrm{D}}^{25}=$ -57.1 $\left(c=1.0\right.$ in $\left.\mathrm{CHCl}_{3}\right) .{ }^{1} \mathrm{H}$ NMR $\left(300 \mathrm{MHz} \mathrm{CDCl}_{3}\right): 1.21(\mathrm{~s}, 9 \mathrm{H}), 2.48(\mathrm{dt}, J=14.1,8.3 \mathrm{~Hz}$, 1H), 2.94-3.01 (m, 1H), $3.87(\mathrm{~d}, J=2.4 \mathrm{~Hz}, 1 \mathrm{H}), 5.20(\mathrm{~m}, 2 \mathrm{H}), 5.83$ (dddd, $J=16.6,10.3,8.3$, $5.9 \mathrm{~Hz}, 1 \mathrm{H}), 6.83(\mathrm{~m}, 1 \mathrm{H}), 7.02(\mathrm{~m}, 1 \mathrm{H}), 7.47(\mathrm{t}, J=7.5 \mathrm{~Hz}, 1 \mathrm{H}), 7.7(\mathrm{~m}, 1 \mathrm{H}), 7.82(\mathrm{dd}, J=7.6$, $1.3 \mathrm{~Hz}, 2 \mathrm{H}), 8.30(\mathrm{~d}, J=15.8 \mathrm{~Hz}, 1 \mathrm{H}) .{ }^{13} \mathrm{C} \mathrm{NMR}\left(75.5 \mathrm{MHz}, \mathrm{CDCl}_{3}\right): 22.7\left(\mathrm{CH}_{3}\right), 44.4\left(\mathrm{CH}_{2}\right)$, $50.4(\mathrm{CH}), 55.8(\mathrm{C}), 87.5(\mathrm{C}), 119.6\left(\mathrm{CH}_{2}\right), 125.4(\mathrm{CH}), 126.2(\mathrm{CH}), 127.9(\mathrm{CH}), 129.7(\mathrm{CH})$, $130.8(\mathrm{CH}), 131.6(\mathrm{C}), 134.2(\mathrm{CH}), 136.1(\mathrm{C}), 138.4(\mathrm{C}) .143 .5(\mathrm{CH})$. HRMS (EI): m/z calcd for $\mathrm{C}_{18} \mathrm{H}_{22} \mathrm{INOS}: 428.0540\left[M+\mathrm{H}^{+}\right]$; found: 428.0540 .

\section{General procedure for the deprotection and Boc protection of sulfonamides $2 a-c$.}

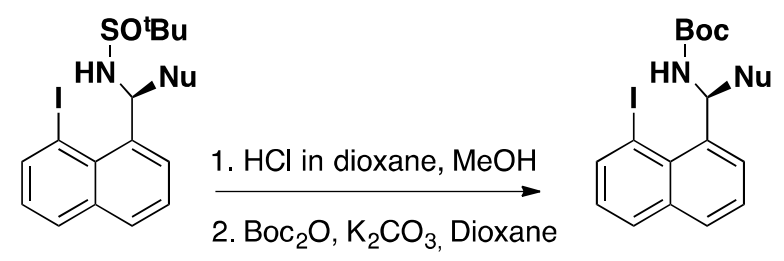

Hydrogen chloride (4.0 equiv, $4 \mathrm{M}$ solution in 1,4-dioxane) was added at $0^{\circ} \mathrm{C}$ to a solution of the corresponding sulfinyl amine 2a-c (1.0 equiv) in $\mathrm{MeOH}(1 \mathrm{M})$. After 20 minutes the deprotection was completed and the solution was concentrated to dryness. The residue was redissolved in $\mathrm{CH}_{2} \mathrm{Cl}_{2}(0.1 \mathrm{M})$ and $2 \mathrm{M} \mathrm{NaOH}$ was added and extracted three times with dichloromethane and the combined organic layers were dried over anhydrous $\mathrm{Na}_{2} \mathrm{SO}_{4}$. Solvents were then removed under reduced pressure. The crude material was dissolved in anhydrous 1,4dioxane $(0.2 \mathrm{M})$ and treated with $\mathrm{K}_{2} \mathrm{CO}_{3}\left(1.1\right.$ equiv) and di-tert-butyl dicarbonate $\left(\mathrm{Boc}_{2} \mathrm{O}\right)(1.1$ equiv) at room temperature until TLC revealed the disappearance of the free amine. The suspension was then filtered through a short pad of Celite ${ }^{\circledR}$ washing with small portions of ethyl acetate. Solvents were removed under reduced pressure and the residue was purified by means of flash chromatography on silica gel (5:1 $n$-hexane:ethyl acetate) affording carbamates 3a-c. 
tert-Butyl (S)-(2,2,2-trifluoro-1-(8-iodonaphthalen-1-yl)ethyl)carbamate (3a)<smiles>CC(C)(C)[NH+](I)c1cccc2cccc(I)c12</smiles>

Compound 3a was synthesized following the general procedure described above starting from 2a (300 mg, $0.66 \mathrm{mmol})$ affording 3a as a colorless oil (125 mg, $42 \%$ yield). $[\alpha]_{\mathrm{D}}^{25}=-46.3\left(c=1.0\right.$ in $\left.\mathrm{CHCl}_{3}\right) \operatorname{er}(92: 8) .{ }^{1} \mathrm{H} \mathrm{NMR}\left(300 \mathrm{MHz}, \mathrm{CDCl}_{3}\right): 1.46(\mathrm{~s}, 9 \mathrm{H}), 5.58$ (br s, 1H), 7.07 (dd, $J=7.3,1.1 \mathrm{~Hz}, 1 \mathrm{H}), 7.52$ (dd, $J=7.8,0.9 \mathrm{~Hz}, 1 \mathrm{H}), 7.70(\mathrm{~m}, 1 \mathrm{H}), 7.83$ (m, $2 \mathrm{H}), 8.38(\mathrm{dd}, J=7.1,1.5 \mathrm{~Hz}, 1 \mathrm{H}) .{ }^{19} \mathrm{~F}$ NMR $\left(282.4 \mathrm{MHz}, \mathrm{CDCl}_{3}\right) \delta-72.6(\mathrm{~s}) .{ }^{13} \mathrm{C}$ NMR $(75.5$ $\left.\mathrm{MHz}, \mathrm{CDCl}_{3}\right): 28.4\left(3 \mathrm{xCH}_{3}\right), 49.6\left(\mathrm{q},{ }^{2} J_{\mathrm{CF}}=31.3 \mathrm{~Hz}, \mathrm{CH}\right), 81.0(\mathrm{C}), 88.0(\mathrm{C}), 123.0(\mathrm{C}), 125.4$ $(\mathrm{CH}), 126.7(\mathrm{CH}), 128.2(\mathrm{CH}), 130.8(\mathrm{CH}), 131.0(\mathrm{C}), 131.5(\mathrm{C}), 131.7(\mathrm{CH}), 136.0(\mathrm{C}), 144.5$ (CH), 154.7 (C). HRMS (EI): m/z calcd for $\mathrm{C}_{17} \mathrm{H}_{17} \mathrm{INOF}_{3}: 452.0329\left[M+\mathrm{H}^{+}\right]$; found: 452.0344.

HPLC (Chiralpak AD, 90:10 hexane $\left./ \mathrm{PrOH}, 1 \mathrm{~mL} \mathrm{~min}^{-1}, 240 \mathrm{~nm}\right) t \mathrm{R}$ (major) $=5.44 \mathrm{~min}$, $t \mathrm{R}(\operatorname{minor})=6.64 \mathrm{~min}$.

\section{Ethyl 3-((S)-(tert-butoxycarbonyl)amino)-3-(8-iodonaphthalen-1-yl)propanoate (3b)}<smiles>CCOC(=O)CC(Nc1cccc2cccc(O)c12)C(=O)O</smiles>

Compound $\mathbf{3 b}$ was synthesized following the general procedure described above starting from $\mathbf{2 b}(300 \mathrm{mg}, 0.64 \mathrm{mmol})$ affording $\mathbf{3 b}$ as a colorless oil (161 $\mathrm{mg}, 54 \%$ yield). $[\alpha]_{\mathrm{D}}^{25}=+18.6\left(c=1.0\right.$ in $\left.\mathrm{CHCl}_{3}\right) \operatorname{er}(99: 1) .{ }^{1} \mathrm{H}$ NMR $\left(300 \mathrm{MHz}, \mathrm{CDCl}_{3}\right): 1.03(\mathrm{t}, J=7.1$ $\mathrm{Hz}, 3 \mathrm{H}), 1.43$ (s, 9H), 2.99 (m, 2H), 3.96 (m, 2H), 5.91 (br s, $1 \mathrm{H}), 7.02$ (dd, $J=7.9,7.5 \mathrm{~Hz}$, $1 \mathrm{H}), 7.26$ (br s, 1H), $7.44(\mathrm{~m}, 1 \mathrm{H}), 7.71(\mathrm{~d}, J=7.8,2 \mathrm{H}), 7.81(\mathrm{dd}, J=8.1,1.1,1 \mathrm{H}), 8.31$ (dd, $J$ = 7.4, 1.3, 1H). ${ }^{13} \mathrm{C}$ NMR (75.5 MHz, $\left.\mathrm{CDCl}_{3}\right): 14.1\left(\mathrm{CH}_{3}\right), 21.6\left(3 \mathrm{x} \mathrm{CH}_{3}\right), 28.5(\mathrm{CH}), 42.0$ $\left(\mathrm{CH}_{2}\right), 60.6\left(\mathrm{CH}_{2}\right), 79.7(\mathrm{C}), 125.5(\mathrm{CH}), 126.0(\mathrm{CH}), 126.3(\mathrm{CH}), 129.9(\mathrm{C}), 130.7(\mathrm{CH}), 131.3$ $(\mathrm{CH}), 136.3(\mathrm{C}), 143.5(\mathrm{CH}), 155.0(\mathrm{C}), 171.0(\mathrm{C})$. HRMS (EI): m/z calcd for $\mathrm{C}_{20} \mathrm{H}_{24} \mathrm{INO}_{4}$ : $470.0823\left[M+\mathrm{H}^{+}\right]$; found: 470.0813 . 
<smiles>C=CCC(NC(=O)OCc1ccccc1)c1cccc2ccccc12</smiles>

Compound 3c was synthesized following the general procedure described above starting from $2 \mathrm{c}$ (300 $\mathrm{mg}, 0.7 \mathrm{mmol}$ ) affording $3 \mathbf{c}$ as a colorless oil (166 mg, $56 \%$ yield). $[\alpha]^{25}=+15.9\left(c=1.0\right.$ in $\left.\mathrm{CHCl}_{3}\right)$ er $(98: 2) .{ }^{1} \mathrm{H} \mathrm{NMR}\left(300 \mathrm{MHz}, \mathrm{CDCl}_{3}\right): 1.44$ (s, 9H), $2.35(\mathrm{~m}$, 1H), $2.83(\mathrm{~m}, 1 \mathrm{H}), 5.02(\mathrm{~m}, 1 \mathrm{H}) 5.15(\mathrm{~m}, 2 \mathrm{H}), 5.77$ (br s, 1H), 7.03 (dd, $J=7.42,0.75 \mathrm{~Hz}, 1 \mathrm{H})$, $7.46(\mathrm{dd}, J=7.8,0.7 \mathrm{~Hz}, 1 \mathrm{H}), 7.65(\mathrm{~m}, 1 \mathrm{H}), 7.72(\mathrm{dd}, J=8.0,1.4 \mathrm{~Hz}, 1 \mathrm{H}), 7.83(\mathrm{dd}, J=8.0$, $1.3 \mathrm{~Hz}, 2 \mathrm{H}), 8.32(\mathrm{dd}, J=7.4,1.4 \mathrm{~Hz}, 1 \mathrm{H}) .{ }^{13} \mathrm{C} \mathrm{NMR}\left(75.5 \mathrm{MHz}, \mathrm{CDCl}_{3}\right): 21.7\left(3 \mathrm{xCH}_{3}\right), 28.5$ $(\mathrm{CH}), 42.9\left(\mathrm{CH}_{2}\right), 56.3(\mathrm{C}), 79.7(\mathrm{C}), 118.5\left(\mathrm{CH}_{2}\right), 125.3(\mathrm{CH}), 125.6(2 \mathrm{xC}), 126.2(\mathrm{CH}), 129.5$ $(\mathrm{CH}), 130.7(\mathrm{CH}), 131.5(\mathrm{C}), 133.9(\mathrm{CH}), 143.4(\mathrm{CH}), 155.3(\mathrm{C})$. HRMS (EI): m/z calcd for $\mathrm{C}_{19} \mathrm{H}_{22} \mathrm{INO}_{2}: 424.0768\left[M+\mathrm{H}^{*}\right]$; found: 424.0763 .

\section{General procedure for the aminocarbonylation of carbamates $3 a-c$.}

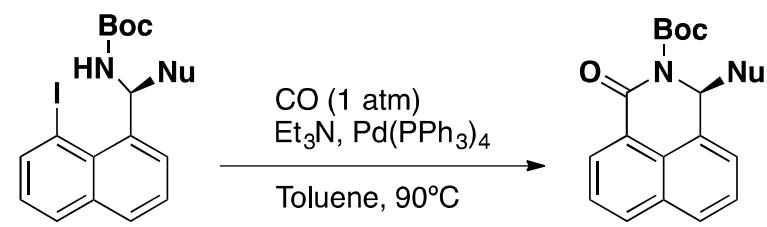

$\mathrm{CO}$ was bubbled for 10 minutes into a suspension of the corresponding carbamate $\mathbf{3}, \operatorname{Pd}\left(\mathrm{PPh}_{3}\right)_{4}$ $(10 \mathrm{~mol} \%)$ and $\mathrm{Et}_{3} \mathrm{~N}$ (4 equiv) in toluene $(0.1 \mathrm{M})$. Then, the reaction mixture was heated at 90 ${ }^{\circ} \mathrm{C}$ under an atmosphere of $\mathrm{CO}$ until TLC revealed the disappearance of the starting material.

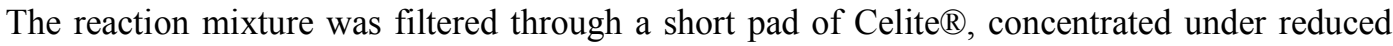
pressure and purified by means of column chromatography on silica gel using mixtures of $n$ hexane: ethyl acetate as eluent.

tert-Butyl (S)-1-oxo-3-(trifluoromethyl)-1H-benzo[de]isoquinoline-2(3H)-carboxylate (4a)<smiles>CC(C)(C)OC(=O)N1C(=O)c2cccc3cccc(c23)[C@@H]1C(F)(F)F</smiles>

Compound 4a was synthesized following the general procedure described above starting from 3a (100 mg, $0.22 \mathrm{mmol}$ ) affording $4 \mathbf{a}$ as a yellowish oil (66 mg, $85 \%$ yield). $[\alpha]_{\mathrm{D}}^{25}=-56.5\left(c=1.0\right.$ in $\left.\mathrm{CHCl}_{3}\right) \operatorname{er}(92: 8) .{ }^{1} \mathrm{H} \mathrm{NMR}\left(300 \mathrm{MHz}, \mathrm{CDCl}_{3}\right): 1.61(\mathrm{~s}, 9 \mathrm{H}), 6.34(\mathrm{dd}$, $J=14.5,7.2 \mathrm{~Hz}, 1 \mathrm{H}), 7.64(\mathrm{~m}, 3 \mathrm{H}), 7.96(\mathrm{dd}, J=7.9,1.5 \mathrm{~Hz}, 1 \mathrm{H}), 8.09(\mathrm{dd}, J=8.1,1.1 \mathrm{~Hz}$, $1 \mathrm{H}), 8.51(\mathrm{dd}, J=7.4,1.3 \mathrm{~Hz}, 1 \mathrm{H}) .{ }^{19} \mathrm{~F}$ NMR $\left(282.4 \mathrm{MHz} . \mathrm{CDCl}_{3}\right) \delta-76.0(\mathrm{~d}, J=8.2 \mathrm{~Hz}) .{ }^{13} \mathrm{C}$ 
$\operatorname{NMR}\left(75.5 \mathrm{MHz}, \mathrm{CDCl}_{3}\right): 28.1\left(3 \mathrm{xCH}_{3}\right), 58.6\left(\mathrm{q},{ }^{2} J_{\mathrm{CF}}=32.4 \mathrm{~Hz}, \mathrm{CH}\right), 84.7(\mathrm{C}), 122.4(\mathrm{C})$, $124.93(\mathrm{CH}), 126.2(\mathrm{CH}), 126.3(\mathrm{CH}), 126.7(\mathrm{CH}), 127.3(\mathrm{CH}), 128.1(\mathrm{C}), 128.7(\mathrm{CH}), 129.1$ $(\mathrm{CH}), 129.4(\mathrm{CH}), 131.3(\mathrm{C}), 132.3(\mathrm{CH}), 133.5(\mathrm{CH}), 146.5(\mathrm{C}), 152.0(\mathrm{C}), 162.5(\mathrm{C})$. HRMS (EI): $\mathrm{m} / \mathrm{z}$ calcd for $\mathrm{C}_{18} \mathrm{H}_{16} \mathrm{~F}_{3} \mathrm{NO}_{3}: 296.0529\left[M-t \mathrm{Bu}+\mathrm{H}^{+}\right]$; found: 296.0536.

HPLC (Chiralpak IC, 95:5 hexane $\left./ \mathrm{PrOH}, 1 \mathrm{~mL} \mathrm{~min}^{-1}, 240 \mathrm{~nm}\right) t \mathrm{R}($ major) $=8.92 \mathrm{~min}$, $t \mathrm{R}($ minor $)=12.17 \mathrm{~min}$.

tert-Butyl (S)-1-(2-ethoxy-2-oxoethyl)-3-oxo-1H-benzo[de]isoquinoline-2(3H)-carboxylate (4b)<smiles>CCOC(=O)C[C@H]1c2cccc3cccc(c23)C(=O)N1C(=O)OCc1ccccc1</smiles>

Compound $\mathbf{4 b}$ was synthesized following the general procedure described above starting from $\mathbf{3 b}(100 \mathrm{mg}, 0.21 \mathrm{mmol})$ affording $\mathbf{4 b}$ as a yellowish oil $(58 \mathrm{mg}, 74 \%$ yield). $[\alpha]_{\mathrm{D}}^{25}=+86.5\left(c=1.0\right.$ in $\left.\mathrm{CHCl}_{3}\right)$ er $(99: 1) .{ }^{1} \mathrm{H} \mathrm{NMR}\left(300 \mathrm{MHz}, \mathrm{CDCl}_{3}\right): 1.10(\mathrm{t}, J=7.2$ $\mathrm{Hz}, 3 \mathrm{H}), 1.61$ (s, 9H), 2.73 (dd, $J=14.8,8.4 \mathrm{~Hz}, 1 \mathrm{H}), 3.03$ (dd, $J=14.8,4.1 \mathrm{~Hz}, 1 \mathrm{H}), 3.98$ (q, $J=7.4 \mathrm{~Hz}, 2 \mathrm{H}), 6.01(\mathrm{dd}, J=8.4,4.2 \mathrm{~Hz}, 1 \mathrm{H}), 7.38(\mathrm{~m}, 1 \mathrm{H}), 7.51(\mathrm{~m}, 2 \mathrm{H}), 7.65(\mathrm{dd}, J=7.2$, $0.9 \mathrm{~Hz}, 1 \mathrm{H}), 7.85(\mathrm{dd}, J=6.8,3.0 \mathrm{~Hz}, 1 \mathrm{H}), 8.08(\mathrm{dd}, J=8.5,1.1 \mathrm{~Hz}, 1 \mathrm{H}), 8.48$ (dd, $J=7.3,1.4$ $\mathrm{Hz}, 1 \mathrm{H}) .{ }^{13} \mathrm{C}$ NMR (75.5 MHz, $\left.\mathrm{CDCl}_{3}\right): 14.1\left(\mathrm{CH}_{3}\right), 28.3\left(3 \mathrm{xCH}_{3}\right), 44.5\left(\mathrm{CH}_{2}\right), 56.0(\mathrm{CH}), 60.9$ $\left(\mathrm{CH}_{2}\right), 83.8(\mathrm{C}), 124.4(\mathrm{CH}), 125.3(\mathrm{C}), 126.4(\mathrm{CH}), 126.5(\mathrm{CH}), 127.4(\mathrm{CH}), 127.5(\mathrm{CH}), 127.8$ $(\mathrm{CH}), 128.7(\mathrm{CH}), 130.4(\mathrm{CH}), 131.4(\mathrm{C}), 132.7(\mathrm{C}), 133.5(\mathrm{CH}), 135.3(\mathrm{CH}), 135.4(\mathrm{CH})$, 153.0 (C), 163.8 (C), 169.9 (C). HRMS (EI): m/z calcd for $\mathrm{C}_{21} \mathrm{H}_{23} \mathrm{NO}_{5}: 270.1125[M-\mathrm{Boc}+$ $\mathrm{H}^{+}$; found: 270.1125 .

HPLC (Chiralpak ODH, 98:2 hexane $\left./ \mathrm{PrOH}, 1 \mathrm{~mL} \mathrm{~min}^{-1}, 240 \mathrm{~nm}\right) t \mathrm{R}($ major$)=17.25 \mathrm{~min}$, $t \mathrm{R}(\operatorname{minor})=45.64 \mathrm{~min}$.

tert-Butyl (S)-1-allyl-3-oxo-1H-benzo[de]isoquinoline-2(3H)-carboxylate (4c)<smiles>C=CCC1c2cccc3cccc(c23)C(=O)N1C(=O)OC(C)(C)C</smiles>

Compound $\mathbf{4 c}$ was synthesized following the general procedure described above starting from $3 \mathbf{c}(100 \mathrm{mg}, 0.24 \mathrm{mmol})$ affording $4 \mathbf{c}$ as a yellowish oil $(31 \mathrm{mg}, 40 \%$ yield). $[\alpha]^{25}=+12.5\left(c=1.0\right.$ in $\left.\mathrm{CHCl}_{3}\right)$ er $(98: 2) .{ }^{1} \mathrm{H} \mathrm{NMR}\left(300 \mathrm{MHz}, \mathrm{CDCl}_{3}\right): 1.61(\mathrm{~s}, 9 \mathrm{H})$, 2.70-2.53 (m, 2H), 4.96-4.83 (m, 2H), $5.49(\mathrm{~d}, J=7.2 \mathrm{~Hz}, 1 \mathrm{H}), 5.64(\mathrm{dd}, J=7.0,5.1 \mathrm{~Hz}, 1 \mathrm{H})$, 
$7.42-7.36(\mathrm{~m}, 1 \mathrm{H}), 7.53(\mathrm{dd}, J=8.3,7.0 \mathrm{~Hz}, 1 \mathrm{H}), 7.64(\mathrm{dd}, J=8.2,7.3,1 \mathrm{H}), 7.83(\mathrm{dd}, J=8.3$, $1.1,1 \mathrm{H}), 8.07(\mathrm{dd}, J=8.1,1.1,1 \mathrm{H}), 8.46(\mathrm{dd}, J=7.3,1.2,1 \mathrm{H}) .{ }^{13} \mathrm{C} \mathrm{NMR}\left(75.5 \mathrm{MHz}, \mathrm{CDCl}_{3}\right)$ : $28.3\left(3 \mathrm{xCH}_{3}\right), 44.5\left(\mathrm{CH}_{2}\right), 59.0(\mathrm{CH}), 83.4(\mathrm{C}), 119.6\left(\mathrm{CH}_{2}\right), 124.2(\mathrm{CH}), 125.9(\mathrm{C}), 126.2$ $(\mathrm{CH}), 126.4(\mathrm{CH}), 126.9(\mathrm{CH}), 127.9(\mathrm{C}), 128.5(\mathrm{CH}), 131.9(\mathrm{C}), 132.6(\mathrm{CH}), 133.3(\mathrm{CH})$, 153.2 (C), 164.2 (C). HRMS (EI): m/z calcd for $\mathrm{C}_{20} \mathrm{H}_{21} \mathrm{NO}_{3}: 324.1591\left[M+\mathrm{H}^{+}\right]$; found: 324.1594.

HPLC (Chiralpak ODH, 98:2 hexane $\left./ i \operatorname{PrOH}, 1 \mathrm{~mL} \mathrm{~min}^{-1}, 240 \mathrm{~nm}\right) t \mathrm{R}$ (major) $=8.15 \mathrm{~min}$, $t \mathrm{R}($ minor $)=10.61 \mathrm{~min}$.

\section{Experimental procedure for the deprotection of carbamate $4 a$.}

TFA (10 equiv) was added to a solution of 5 in DCM $(0.1 \mathrm{M})$ at $0{ }^{\circ} \mathrm{C}$ and the reaction mixture was refluxed until TLC analysis revealed complete consumption of the starting material. The reaction is then quenched with saturated $\mathrm{NaHCO} 3(\mathrm{aq})$ and extracted with DCM (3 times). The combined organic layers are dried over anhydrous $\mathrm{Na} 2 \mathrm{SO} 4$, concentrated under reduced pressure and the crude material purified by column chromatography on silica gel using mixtures of hexane and ethyl acetate as eluent.

\section{(S)-3-(trifluoromethyl)-2,3-dihydro-1H-benzo[de]isoquinolin-1-one (IV)}<smiles>O=C1N[C@H](C(F)(F)F)c2cccc3cccc1c23</smiles>

Compound IV was synthesized following the general procedure described above starting from 4a (100 mg, $0.22 \mathrm{mmol})$ affording IV as a yellowish oil (70 $\mathrm{mg}, 88 \%$ yield). $[\alpha]_{\mathrm{D}}^{25}=-102.4\left(c=1.0\right.$ in $\left.\mathrm{CHCl}_{3}\right)$ er $(92: 8) .{ }^{1} \mathrm{H} \mathrm{NMR}\left(300 \mathrm{MHz}, \mathrm{CDCl}_{3}\right): 5.38(\mathrm{qd}, J=13.9$, $5.4 \mathrm{~Hz}, 1 \mathrm{H}), 7.58(\mathrm{~m}, 2 \mathrm{H}), 7.65(\mathrm{dd}, J=8.4,7.2 \mathrm{~Hz}, 1 \mathrm{H}), 7.95(\mathrm{dd}, J=7.8,2.4 \mathrm{~Hz}, 1 \mathrm{H}), 8.07$ $(\mathrm{dd}, J=8.2,1.1 \mathrm{~Hz}, 1 \mathrm{H}), 8.43(\mathrm{dd}, J=7.1,0.9 \mathrm{~Hz}, 1 \mathrm{H}) .{ }^{19} \mathrm{~F} \mathrm{NMR}\left(282.4 \mathrm{MHz} . \mathrm{CDCl}_{3}\right) \delta-78.5$ $(\mathrm{d}, J=6.00 \mathrm{~Hz}) .{ }^{13} \mathrm{C} \mathrm{NMR}\left(75.5 \mathrm{MHz}, \mathrm{CDCl}_{3}\right): 57.7\left(\mathrm{q},{ }^{2} J_{\mathrm{CF}}=31.1 \mathrm{~Hz}, \mathrm{CH}\right), 121.6(\mathrm{C}), 123.2$ (C), $125.9\left(\mathrm{q},{ }^{1} J_{\mathrm{CF}}=283.3 \mathrm{~Hz}, \mathrm{CF}_{3}\right), 126.1(\mathrm{CH}), 126.6(\mathrm{CH}), 126.9(\mathrm{CH}), 127.7(\mathrm{CH}), 129.1$ (C), $129.2(\mathrm{CH}), 132.6(\mathrm{C}), 132.9(\mathrm{CH}), 165.0(\mathrm{C})$.

HPLC (Chiralpak IC, 85:15 hexane $\left./ \mathrm{PrOH}, 1 \mathrm{~mL} \mathrm{~min}^{-1}, 240 \mathrm{~nm}\right) t \mathrm{R}($ major) $=9.49 \mathrm{~min}$, $t \mathrm{R}($ minor $)=13.77 \mathrm{~min}$. 
General procedure for the Buchwald-Hartwig amination of carbamates $3 a, b$.

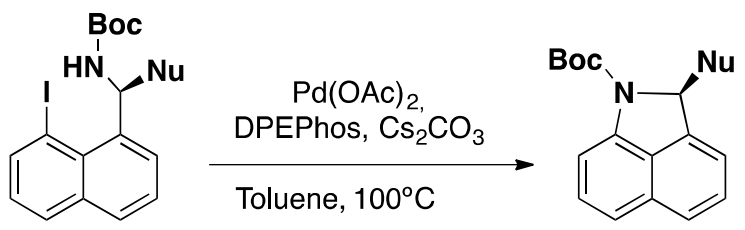

A suspension of the corresponding carbamate 3, Pd(OAc) 2 (5 mol\%), DPEPhos (10 mol\%) and $\mathrm{Cs}_{2} \mathrm{CO}_{3}$ (1.4 equiv) in toluene $(0.1 \mathrm{M})$ was heated at $120{ }^{\circ} \mathrm{C}$ for $4 \mathrm{~h}$ under microwave irradiation. The reaction mixture was filtered through a short pad of Celite ${ }^{\circledR}$, concentrated under reduced pressure and purified by means of column chromatography on silica gel using mixtures of hexane: ethyl acetate (5:1) as eluent.

tert-Butyl (R)-2-(trifluoromethyl)benzo[cd]indole-1(2H)-carboxylate (5a)<smiles>O=C(c1ccccc1)N1c2cccc3cccc(c23)C1C(F)(F)F</smiles>

Compound 5a was synthesized following the general procedure described above starting from 3a (100 mg, $0.22 \mathrm{mmol})$ affording $\mathbf{5 a}$ as a yellow oil $(28 \mathrm{mg}, 40 \%$ yield $)$. $[\alpha]^{25}=$ -25.5 (c 1.0, $\left.\mathrm{CHCl}_{3}\right) .{ }^{1} \mathrm{H}$ NMR (300 MHz, $\left.\mathrm{CDCl}_{3}\right) \delta 1.56$ (s, 9H), 5.98 (br s, 1H), $7.46(\mathrm{~m}, 5 \mathrm{H})$, $7.70(\mathrm{~m}, 1 \mathrm{H}) .{ }^{19} \mathrm{~F}$ NMR $\left(282.4 \mathrm{MHz}, \mathrm{CDCl}_{3}\right) \delta-75.1\left(\mathrm{~s}, \mathrm{CF}_{3}\right) .{ }^{13} \mathrm{C} \mathrm{NMR}\left(75.5 \mathrm{MHz}, \mathrm{CDCl}_{3}\right) \delta$ $28.14\left(3 \mathrm{x} \mathrm{CH}_{3}\right), 65.17(\mathrm{CH}, \mathrm{q}, J=33.3), 82.94(\mathrm{C}), 109.51(\mathrm{CH}), 119.07(\mathrm{CH}), 119.36(\mathrm{CH})$, $123.78\left(\mathrm{CF}_{3}, \mathrm{q}, J=282.2\right), 125.31(\mathrm{CH}), 127.95(\mathrm{CH}), 129.12(\mathrm{CH}), 129.81(\mathrm{C}), 130.75(\mathrm{C})$, 131.02 (C), 142.40 (C), 151.95 (C). HRMS (EI) calcd for $\mathrm{C}_{17} \mathrm{H}_{16} \mathrm{~F}_{3} \mathrm{NO}_{2}$ [M- $\left.t \mathrm{Bu}+\mathrm{H}^{+}\right]$: 268.0580 , found 268.0587 .

HPLC (Chiralpak ODH, 90:10 hexane/iPrOH, $\left.1 \mathrm{~mL} \mathrm{~min}^{-1}, 240 \mathrm{~nm}\right) t \mathrm{R}($ major) $=4.52 \mathrm{~min}$, $t \mathrm{R}($ minor $)=6.87 \mathrm{~min}$.

tert-Butyl (R)-2-(2-ethoxy-2-oxoethyl)benzo[cd]indole-1(2H)-carboxylate (5b).<smiles>CCOC(=O)CC1c2cccc3cccc(c23)N1C(=O)OC(C)(C)C</smiles>

Compound $\mathbf{5 b}$ was synthesized following the general procedure described above starting from $\mathbf{3 b}$ (100 mg, $0.22 \mathrm{mmol}$ ) affording $\mathbf{5 b}$ as a yellow oil (31 $\mathrm{mg}, 43 \%$ yield). 
$[\alpha]^{25}=+62.3\left(c 1.0, \mathrm{CHCl}_{3}\right) .{ }^{1} \mathrm{H} \mathrm{NMR}\left(300 \mathrm{MHz}, \mathrm{CDCl}_{3}\right) \delta 1.16(\mathrm{~s}, 3 \mathrm{H}), 1.65(\mathrm{~s}, 9 \mathrm{H}), 2.79(\mathrm{dd}$, $J=8.9,15.5 \mathrm{~Hz}, 1 \mathrm{H}), 3.40($ br s, $1 \mathrm{H}), 4.14(\mathrm{q}, J=7.5 \mathrm{~Hz}, 2 \mathrm{H}), 5.88($ br s, $1 \mathrm{H}), 7.40(\mathrm{~m}, 5 \mathrm{H})$, $7.65(\mathrm{dd}, J=1.8,0.9 \mathrm{~Hz}, 1 \mathrm{H}) .{ }^{13} \mathrm{C} \mathrm{NMR}\left(75.5 \mathrm{MHz}, \mathrm{CDCl}_{3}\right) \delta 14.1\left(\mathrm{CH}_{3}\right), 28.4\left(3 \mathrm{xCH}_{3}\right), 60.6$ $\left(\mathrm{CH}_{2}\right), 62.3(\mathrm{CH}), 108.0(\mathrm{CH}), 108.9(\mathrm{C}), 117.2(\mathrm{CH}), 118.1(\mathrm{CH}), 118.9(\mathrm{C}), 123.7(\mathrm{CH}), 128.1$ $(\mathrm{CH}), 129.0(\mathrm{CH}), 131.3(\mathrm{C}), 139.1(\mathrm{C}), 170.5(\mathrm{C})$. HRMS (EI) calcd for $\mathrm{C}_{20} \mathrm{H}_{23} \mathrm{NO}_{4}\left[\mathrm{M}+\mathrm{H}^{+}\right]$: 342.1700 , found 342.1697 .

HPLC (Chiralpak ODH, 98:2 hexane $\left./ i \operatorname{PrOH}, 1 \mathrm{~mL} \mathrm{~min}^{-1}, 240 \mathrm{~nm}\right) t \mathrm{R}$ (major) $=6.84 \mathrm{~min}$, $t \mathrm{R}($ minor $)=10.45 \mathrm{~min}$.

General procedure for the intramolecular Heck reaction of $\mathrm{N}$-tert-Butanesulfinyl Imine $2 c$.

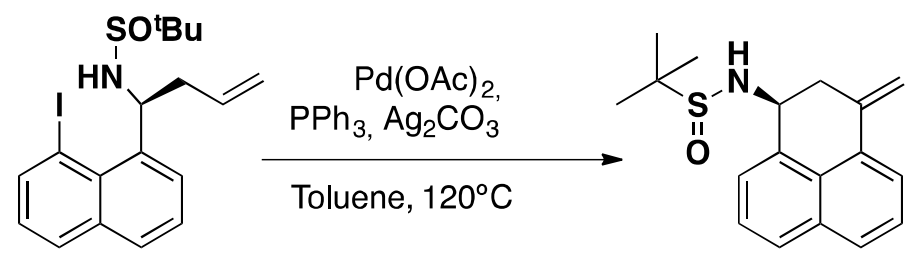

A suspension of the corresponding $N$-tert-butanesulfinyl imine $2 \mathbf{c}, \mathrm{Pd}(\mathrm{OAc})_{2}(10 \mathrm{~mol} \%), \mathrm{PPh}_{3}$ $(15 \mathrm{~mol} \%)$ and $\mathrm{Ag}_{2} \mathrm{CO}_{3}$ (1 equiv) in toluene $(0.1 \mathrm{M})$ was heated at $120{ }^{\circ} \mathrm{C}$ for $1 \mathrm{~h}$ under microwave irradiation. The reaction mixture was filtered through a short pad of Celite ${ }^{\circledR}$, concentrated under reduced pressure and purified by means of column chromatography on silica gel using mixtures of hexane: ethyl acetate (10:1) as eluent.

\section{2-Methyl-N-((R)-3-methylene-2,3-dihydro-1H-phenalen-1-yl)propane-2-sulfinamide (6).}<smiles>C=C1C[C@H](NS(=O)(=O)OCCCC)c2cccc3cccc1c23</smiles>

Compound $\mathbf{6}$ was synthesized following the general procedure described above starting from $2 \mathrm{c}$ (100 $\mathrm{mg}, 0.24 \mathrm{mmol})$ affording 6 as a yellow oil (31 $\mathrm{mg}, 44 \%$ yield). $[\alpha]^{25}{ }_{\mathrm{D}}=+23.5\left(c 1.0, \mathrm{CHCl}_{3}\right) .{ }^{1} \mathrm{H}$ NMR $\left(300 \mathrm{MHz}, \mathrm{CDCl}_{3}\right) \delta 1.16(\mathrm{~s}, 9 \mathrm{H}), 2.99(\mathrm{dd}, J=13.1,6.4$ $\mathrm{Hz}, 1 \mathrm{H}), 3.17$ (dd, $J=13.1,4.2 \mathrm{~Hz}, 1 \mathrm{H}), 3.62$ (d, $J=9.4 \mathrm{~Hz}, 1 \mathrm{H}), 4.86(\mathrm{~m}, 1 \mathrm{H}), 5.31$ (d, $J=1.3$ $\mathrm{Hz}, 1 \mathrm{H}), 5.74(\mathrm{~s}, 1 \mathrm{H}), 7.51(\mathrm{~m}, 3 \mathrm{H}), 7.73$ (dd, $J=7.4,1.4 \mathrm{~Hz}, 1 \mathrm{H}), 7.81$ (ddd, $J=7.3,5.4,1.2$ $\mathrm{Hz}, 2 \mathrm{H}) .{ }^{13} \mathrm{C} \mathrm{NMR}\left(75.5 \mathrm{MHz}, \mathrm{CDCl}_{3}\right) \delta 22.8\left(3 \mathrm{xCH}_{3}\right), 41.2\left(\mathrm{CH}_{2}\right), 56.4(\mathrm{C}), 56.8(\mathrm{CH}), 113.3$ $\left(\mathrm{CH}_{2}\right), 121.4(\mathrm{CH}), 125.0(\mathrm{CH}), 125.8(\mathrm{CH}), 126.0(\mathrm{CH}), 128.0(\mathrm{CH}), 128.3(\mathrm{CH}), 133.5(\mathrm{C})$, 133.7 (C), 135.7 (C), 140.3 (C). HRMS (EI) calcd for $\mathrm{C}_{18} \mathrm{H}_{21} \mathrm{NOS}\left[\mathrm{M}+\mathrm{H}^{+}\right]$: 299.1098, found 299.1100. 
tert-Butyl (S)-(3-methylene-2,3-dihydro-1H-phenalen-1-yl)carbamate (6').<smiles>C=C1C[C@H](NC(C)(C)C)c2cccc3cccc1c23</smiles>

Compound 6' was synthesized following the general procedure described above starting from 3c (100 mg, $0.24 \mathrm{mmol})$ affording 6' as a yellow oil (23 mg, 33\% yield). (c 1.0, $\mathrm{CHCl}_{3}$ ). ${ }^{1} \mathrm{H} \mathrm{NMR}\left(300 \mathrm{MHz}, \mathrm{CDCl}_{3}\right) \delta 1.45(\mathrm{~s}, 9 \mathrm{H}), 2.82(\mathrm{dd}, J=13.2,5.8 \mathrm{~Hz}, 1 \mathrm{H}), 2.95$ (m, 1H), $4.84(\mathrm{~d}, J=9.0 \mathrm{~Hz}, 1 \mathrm{H}), 5.25(\mathrm{~m}, 2 \mathrm{H}), 5.72(\mathrm{~s}, 1 \mathrm{H}), 7.48(\mathrm{~m}, 2 \mathrm{H}), 7.60(\mathrm{~m}, 1 \mathrm{H}), 7.73$ $(\mathrm{dd}, J=7.3,1.4 \mathrm{~Hz}, 1 \mathrm{H}), 7.80(\mathrm{ddd}, J=10.9,8.2,1.2 \mathrm{~Hz}, 2 \mathrm{H}) .{ }^{13} \mathrm{C} \mathrm{NMR}\left(75.5 \mathrm{MHz}, \mathrm{CDCl}_{3}\right) \delta$ $28.6\left(3 \mathrm{xCH}_{3}\right), 38.9\left(\mathrm{CH}_{2}\right), 50.1(\mathrm{CH}), 79.6(\mathrm{C}), 100.1(\mathrm{C}), 112.6\left(\mathrm{CH}_{2}\right), 121.4(\mathrm{CH}), 125.1$ $(\mathrm{CH}), 125.9(\mathrm{CH}), 126.0(\mathrm{CH}), 127.8(\mathrm{CH}), 128.4(\mathrm{CH}), 133.4(\mathrm{C}), 133.7(\mathrm{C}), 135.7(\mathrm{C}), 140.8$ (C), 155.3 (C). HRMS (EI) calcd for $\mathrm{C}_{19} \mathrm{H}_{21} \mathrm{NO}_{2}\left[\mathrm{M}^{+}+\mathrm{H}\right]$ : 296.1281, found 296.1287.

\section{Experimental procedure for the Suzuki coupling.}

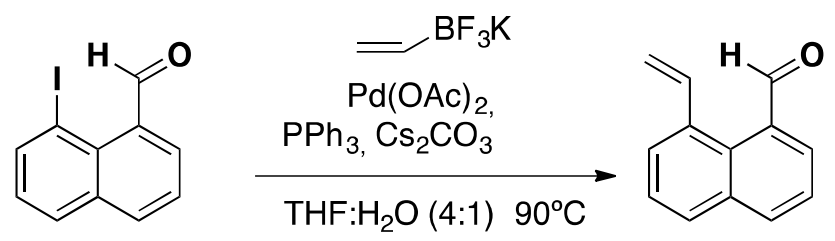

To a solution of 1 and potassium vinyltrifluoroborate $(0.5 \mathrm{mmol}, 1$ equiv) in a 9:1 toluene/water mixture $(5 \mathrm{ml}, 0.1 \mathrm{M})$ in a microwave vial, $\mathrm{Pd}(\mathrm{dba})_{2}(0.025 \mathrm{mmol}, 5 \mathrm{~mol} \%)$, phosphine $(0.025$ mmol, $5 \mathrm{~mol} \%)$ and $\mathrm{K}_{3} \mathrm{PO}_{4}\left(1.5 \mathrm{mmol}, 3\right.$ equiv) were added. The solution was heated at $100^{\circ} \mathrm{C}$ in a microwave oven for 4 hours. After this time the reaction mixture was quenched with saturated $\mathrm{NH}_{4} \mathrm{Cl}$ aq. And extracted three times with AcOEt. The combined organic layers were dried over anhydrous $\mathrm{Na}_{2} \mathrm{SO}_{4}$. Solvents were removed under reduced pressure and the residue was purified by means of flash chromatography on silica gel.

\section{8-Vinyl-1-naphthaldehyde (7).}<smiles>C=Cc1cccc2cccc(C=O)c12</smiles>

Compound 7 was synthesized following the general procedure described above starting from 1 (500 mg, $1.77 \mathrm{mmol})$ affording 7 as a colorless oil $\left(161 \mathrm{mg}, 50 \%\right.$ yield). ${ }^{1} \mathrm{H}$ $\operatorname{NMR}\left(300 \mathrm{MHz}, \mathrm{CDCl}_{3}\right) \delta 5.34(\mathrm{dd}, J=10.8,1.4 \mathrm{~Hz}, 1 \mathrm{H}), 5.66(\mathrm{dd}, J=17.1,1.3 \mathrm{~Hz}, 1 \mathrm{H}), 7.11$ (ddd, $J=17.1,10.7,0.7 \mathrm{~Hz}, 1 \mathrm{H}), 7.44(\mathrm{~m}, 2 \mathrm{H}), 7.56(\mathrm{~m}, 1 \mathrm{H}), 7.75(\mathrm{dd}, J=18.1,1.3 \mathrm{~Hz}, 1 \mathrm{H})$, 
$7.92(\mathrm{~m}, 2 \mathrm{H}), 10.77(\mathrm{~d}, J=0.7 \mathrm{~Hz}, 1 \mathrm{H}) .{ }^{13} \mathrm{C} \mathrm{NMR}\left(75.5 \mathrm{MHz}, \mathrm{CDCl}_{3}\right) \delta 117.1\left(\mathrm{CH}_{2}\right), 125.3$ $(\mathrm{CH}), 126.5(\mathrm{CH}), 128.3(\mathrm{CH}), 129.0(\mathrm{CH}), 129.2(\mathrm{CH}), 129.8(\mathrm{C}), 134.4(\mathrm{C}), 134.6(\mathrm{CH})$, $135.0(\mathrm{C}), 135.7(\mathrm{C}), 139.0(\mathrm{CH}), 193.4(\mathrm{C})$. HRMS (EI) calcd for $\mathrm{C}_{13} \mathrm{H}_{10} \mathrm{O}\left[\mathrm{M}^{+}-\mathrm{H}\right]: 181.0648$, found 181.0639 .

\section{Experimental procedure for the one-pot condensation/allylation/RCM sequence.}

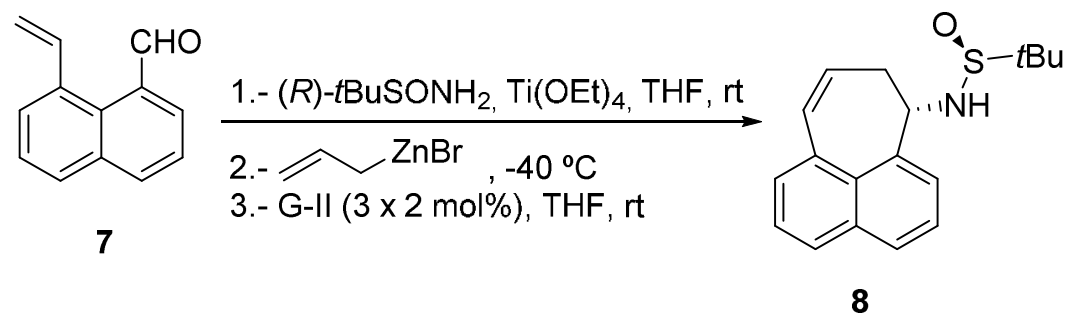

A solution of the corresponding aldehyde $\left(0.5 \mathrm{mmol}, 1.0\right.$ equiv) and $\mathrm{Ti}(\mathrm{OEt})_{4}$ (2.5 mmol, 4.0 equiv) in dichloromethane $(5 \mathrm{ml}, 0.1 \mathrm{M})$ was stirred for 5 minutes at room temperature. To the resulting solution $(R)-N$-(tert-butanesulfinyl)amine (0.6 mmol, 1.2 equiv) was added and the mixture was stirred at room temperature for 12 hours. After this time, saturated aqueous $\mathrm{NaHCO}_{3}$ was added until white titanium salts precipitated. The suspension was filtered through a short pad of Celite ${ }^{\circledR}$ and washed with small portions of dichloromethane. The filtrate was extracted with ethyl acetate and the combined organic layers were washed with brine, dried over anhydrous $\mathrm{Na}_{2} \mathrm{SO}_{4}$ and concentrated under vacuum. The crude product was used in the next step without purification.

Subsequently, a $1 \mathrm{M}$ solution of allylzinc bromide in THF was prepared by stirring allyl bromide $(0.19 \mathrm{~mL}, 2.25 \mathrm{mmol}, 1.0$ equiv) and activated $\mathrm{Zn}(0.5 \mathrm{~g}, 7.6$ mmol, 3.6 equiv) in anhydrous THF $(2.25 \mathrm{ml}, 1 \mathrm{M})$ at $55^{\circ} \mathrm{C} .1 .1$ equiv $(0.33 \mathrm{mmol}, 0.33$ $\mathrm{mL}$ ) of this freshly prepared solution were added to the residue obtained from the previous step dissolved in THF $(3 \mathrm{ml}, 0.1 \mathrm{M})$ at $-40{ }^{\circ} \mathrm{C}$. After stirring for 30 minutes (until TLC revealed the disappearance of the starting material) at $-40{ }^{\circ} \mathrm{C}$ the reaction mixture was quenched with saturated $\mathrm{NH}_{4} \mathrm{Cl}$ aquous and extracted three times with AcOEt, dried over anhydrous $\mathrm{Na}_{2} \mathrm{SO}_{4}$ and concentrated under vacuum.

Finally, to a solution of the corresponding allylated compound in DCM (2 ml, $0.1 \mathrm{M}) 2^{\text {nd }}$ generation Grubbs catalyst $(2 \mathrm{~mol} \%$ ) was added and the mixture was stirred at room temperature for 12 hours. $2^{\text {nd }}$ generation Grubbs catalyst $(2 \mathrm{~mol} \%$ ) was added 
again and stirred at room temperature for a further 10 hours. Once again, a third portion of $2^{\text {nd }}$ generation Grubbs catalyst $(2 \mathrm{~mol} \%)$ was added followed by 6 extra hours stirring. After that time, the reaction mixture was concentrated under vacuum and purified by means of flash chromatography on silica gel employing mixtures of $n$ hexane: ethyl acetate as eluents.

N-(7,8-Dihydrocyclohepta[de]naphthalen-7-yl)-2-methylpropane-2-sulfinamide (8).<smiles>CCCCS(=O)NC1CC=Cc2cccc3cccc1c23</smiles>

Compound $\mathbf{8}$ was synthesized following the general procedure described above starting from $7(80 \mathrm{mg}, 0.28 \mathrm{mmol})$ affording as a brown oil $(25 \mathrm{mg}, 30 \%$ yield $)$. $[\alpha]^{25}=$ +9 (c 1.0, $\left.\mathrm{CHCl}_{3}\right) .{ }^{1} \mathrm{H} \mathrm{NMR}\left(300 \mathrm{MHz}, \mathrm{CDCl}_{3}\right) \delta 0.93(\mathrm{~s}, 9 \mathrm{H}), 2.95(\mathrm{~m}, 1 \mathrm{H}), 3.11(\mathrm{~m}, 1 \mathrm{H}), 4.07$ (d, $J=7.8 \mathrm{~Hz}, 1 \mathrm{H}), 5.00(\mathrm{~m}, 1 \mathrm{H}), 5.96$ (dddd, $J=11.6,8.2,3.4,1.1 \mathrm{~Hz}, 1 \mathrm{H}), 6.84$ (dd, $J=11.9$, $2.9 \mathrm{~Hz}, 1 \mathrm{H}), 7.43(\mathrm{~m}, 4 \mathrm{H}), 7.80(\mathrm{~m}, 2 \mathrm{H}) .{ }^{13} \mathrm{C} \mathrm{NMR}\left(75.5 \mathrm{MHz}, \mathrm{CDCl}_{3}\right) \delta 22.3\left(3 \mathrm{xCH}_{3}\right), 35.6$ $\left(\mathrm{CH}_{2}\right), 55.6(\mathrm{C}), 61.8(\mathrm{CH}), 125.4(\mathrm{CH}), 126.3(\mathrm{CH}), 128.4(\mathrm{CH}), 129.2(\mathrm{C}), 129.4(\mathrm{CH}), 129.6$ $(\mathrm{CH}), 131.8(\mathrm{CH}), 133.9(\mathrm{C}), 134.5(\mathrm{CH}), 135.7(\mathrm{C}), 139.6(\mathrm{C})$. HRMS (EI) calcd for $\mathrm{C}_{18} \mathrm{H}_{21} \mathrm{NOS}\left[\mathrm{M}+\mathrm{H}^{+}\right]: 300.1417$, found 300.1417 .

\section{General procedure for the Sonogashira cross-coupling reaction.}
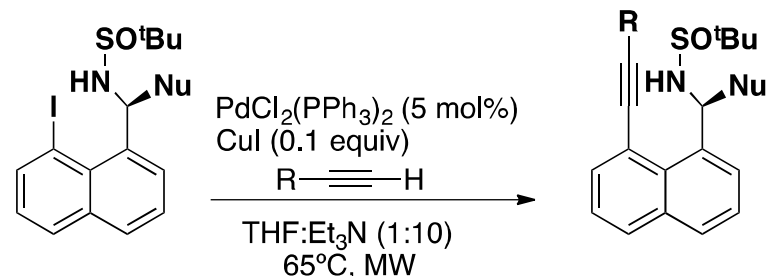

To a solution of the corresponding compound 2a-c (1 equiv) and $\mathrm{PdCl}_{2}\left(\mathrm{PPh}_{3}\right)_{2}(0.05$ equiv) in $\mathrm{THF}: \mathrm{Et}_{3} \mathrm{~N}(1: 10)$ the corresponding acetylene (1.2 equiv) was added and the mixture was stirred for $10 \mathrm{~min}$ at room temperature. After that time, $\mathrm{CuI}(0.1$ equiv) was added in one portion into the reaction mixture, and the resulting brown suspension was then stirred at $65^{\circ} \mathrm{C}$ for $2 \mathrm{~h}$ under microwave irradiation. The reaction mixture was then diluted with diethyl ether and the resulting dark solution was filtered through a pad of silica gel. The filtrate was concentrated under vacuum and purified via flash column chromatography on silica employing mixtures of $n$ hexane: ethyl acetate (3:1) as the eluent to afford compounds 10aa-10cc. 


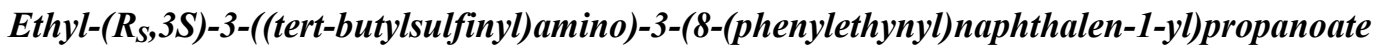
(10ba).

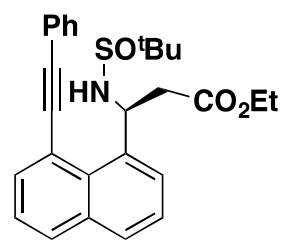

Compound 10ba was synthesized following the general procedure described above starting from $\mathbf{2 b}(300 \mathrm{mg}, 0.64 \mathrm{mmol})$ affording 10ba as a yellow-green oil (241 $\mathrm{mg}, 85$ \% yield). $[\alpha]_{\mathrm{D}}^{25}=-27.3\left(c=1.0\right.$ in $\left.\mathrm{CHCl}_{3}\right) .{ }^{1} \mathrm{H}$ NMR $\left(300 \mathrm{MHz}, \mathrm{CDCl}_{3}\right): 0.98(t, J=7.3 \mathrm{~Hz}$, $3 \mathrm{H}), 1.27$ (s, 9H), 3.05 (dd, $J=15.9,7.4 \mathrm{~Hz}, 1 \mathrm{H}), 3.23$ (dd, $J=16.0,4.8 \mathrm{~Hz}, 1 \mathrm{H}), 3.83(\mathrm{~m}, 2 \mathrm{H})$, $5.36(\mathrm{~d}, J=5.4 \mathrm{~Hz}, 1 \mathrm{H}), 7.42(\mathrm{~m}, 5 \mathrm{H}), 7.70(\mathrm{~m}, 2 \mathrm{H}), 7.80(\mathrm{ddd}, J=4.8,3.3,1.4 \mathrm{~Hz}, 2 \mathrm{H}), 7.87$ (m, 2H). ${ }^{13} \mathrm{C}$ NMR (75.5 MHz, $\left.\mathrm{CDCl}_{3}\right): 13.8\left(\mathrm{CH}_{3}\right), 22.7\left(3 \mathrm{xCH}_{3}\right), 42.4\left(\mathrm{CH}_{2}\right), 51.3(\mathrm{CH}), 55.8$ (C), $60.6\left(\mathrm{CH}_{2}\right), 91.2(\mathrm{C}), 94.3(\mathrm{C}), 118.2(\mathrm{C}), 123.1(\mathrm{C}), 124.8(\mathrm{CH}), 125.5(\mathrm{CH}), 126.4(\mathrm{CH})$, $128.4(\mathrm{CH}), 128.5(2 \times \mathrm{CH}), 129.5(\mathrm{CH}), 130.6(\mathrm{CH}), 131.5(2 \times \mathrm{CH}), 134.8(\mathrm{C}), 135.6(2 \times \mathrm{C})$, $138.5(\mathrm{CH}), 171.9(\mathrm{C})$. HRMS (EI): $\mathrm{m} / \mathrm{z}$ calcd for $\mathrm{C}_{27} \mathrm{H}_{29} \mathrm{NO}_{3} \mathrm{~S}: 448.1946\left[M^{+}+1\right]$; found: 448.1941.

2-Methyl-N-(( $\left.\boldsymbol{R}_{S}, S\right)-1-(8-($ phenylethynyl)naphthalen-1-yl)but-3-en-1-yl)propane-2sulfinamide (10ca).

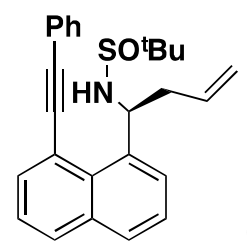

Compound 10ca was synthesized following the general procedure described above starting from $\mathbf{2 c}(300 \mathrm{mg}, 0.71 \mathrm{mmol})$ affording $10 \mathrm{ca}$ as a yellow-green oil $(239 \mathrm{mg}, 83$

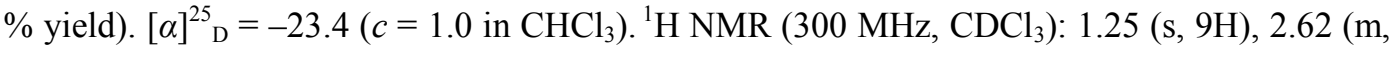
1H), $3.02(\mathrm{~m}, 1 \mathrm{H}), 3.93(\mathrm{~d}, J=3.8 \mathrm{~Hz}, 1 \mathrm{H}), 5.17$ (m, 2H), 5.73 (dddd, $J=17.8,14.3,7.9,6.4$ $\mathrm{Hz}, 1 \mathrm{H}), 7.07$ (dt, $J=8.1,4.1 \mathrm{~Hz}, 1 \mathrm{H}), 7.42(\mathrm{~m}, 5 \mathrm{H}), 7.72(\mathrm{~m}, 2 \mathrm{H}), 7.79(\mathrm{~m}, 2 \mathrm{H}), 7.87$ (m, 2H). ${ }^{13} \mathrm{C}$ NMR (75.5 MHz, $\left.\mathrm{CDCl}_{3}\right): 22.7\left(3 \mathrm{xCH}_{3}\right), 43.9\left(\mathrm{CH}_{2}\right), 52.3(\mathrm{CH}), 55.8(\mathrm{C}), 91.4(\mathrm{C}), 94.3$ (C), $118.4(\mathrm{C}), 119.5\left(\mathrm{CH}_{2}\right), 123.3(\mathrm{C}), 124.7(\mathrm{CH}), 125.4(\mathrm{CH}), 126.2(\mathrm{CH}), 128.4(\mathrm{CH}), 128.5$ $(\mathrm{CH}), 129.0(\mathrm{CH}), 129.8(\mathrm{C}), 130.5(\mathrm{CH}), 131.4(2 \times \mathrm{CH}), 134.3(\mathrm{CH}), 134.8(\mathrm{C}), 135.5(\mathrm{CH})$, 139.9 (C). HRMS (EI): m/z calcd for $\mathrm{C}_{26} \mathrm{H}_{27} \mathrm{NOS}: 402.1886\left[M+\mathrm{H}^{+}\right]$; found: 402.1878. 
2-Methyl-N-((S)-2,2,2-trifluoro-1-(8-((2-methoxyphenyl)ethynyl)naphthalen-1-

yl)ethyl)propane-2-sulfinamide (10ab).

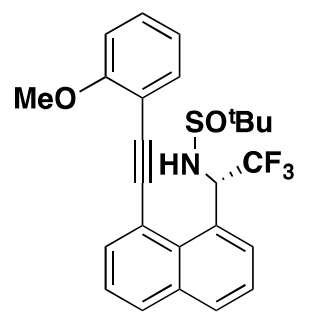

Compound 10ab was synthesized following the general procedure described above starting from 2a (300 mg, $0.66 \mathrm{mmol}$ ) affording 10ab as a yellow-green oil (66 $\mathrm{mg}, 22 \%$ yield). $[\alpha]^{25}=-1.07\left(c=1.0\right.$ in $\left.\mathrm{CHCl}_{3}\right) .{ }^{1} \mathrm{H}$ NMR $\left(300 \mathrm{MHz}, \mathrm{CDCl}_{3}\right): 1.07(\mathrm{~s}, 9 \mathrm{H})$, $3.96(\mathrm{~s}, 3 \mathrm{H}), 4.59$ (d, $J=7.2 \mathrm{~Hz}, 1 \mathrm{H}), 6.96$ (dd, $J=8.4,1.1 \mathrm{~Hz}, 1 \mathrm{H}), 7.01$ (dd, $J=7.5,1.0 \mathrm{~Hz}$, $1 \mathrm{H}), 7.35(\mathrm{~m}, 1 \mathrm{H}), 7.49(\mathrm{~m}, 2 \mathrm{H}), 7.61(\mathrm{~m}, 1 \mathrm{H}), 7.85(\mathrm{~m}, 4 \mathrm{H}), 7.95(\mathrm{dd}, J=7.3,1.5 \mathrm{~Hz}, 1 \mathrm{H}) .{ }^{19} \mathrm{~F}$ NMR (282.4 MHz, $\left.\mathrm{CDCl}_{3}\right) \delta-71.0\left(\mathrm{~d}, J=8.1 \mathrm{~Hz}, \mathrm{CF}_{3}\right) .{ }^{13} \mathrm{C} \mathrm{NMR}\left(75.5 \mathrm{MHz}, \mathrm{CDCl}_{3}\right): 22.1$ $\left(3 \mathrm{xCH}_{3}\right), 55.2\left(\mathrm{q},{ }^{2} J_{\mathrm{CF}}=29.8 \mathrm{~Hz}, \mathrm{CH}\right), 55.4\left(\mathrm{CH}_{3}\right), 56.5(\mathrm{C}), 92.1(\mathrm{C}), 94.6(\mathrm{C}), 110.6(\mathrm{CH})$, $112.2(\mathrm{C}), 118.6(\mathrm{C}), 120.7(\mathrm{CH}), 123.7\left(\mathrm{CF}_{3}\right), 125.2(\mathrm{CH}), 125.3(\mathrm{CH}), 127.4(\mathrm{CH}), 129.9(\mathrm{C})$, $130.1(\mathrm{CH}), 130.6(\mathrm{CH}), 131.1(\mathrm{CH}), 133.4(\mathrm{CH}), 134.8(\mathrm{C}), 136.4(\mathrm{CH}), 159.8(\mathrm{C})$. HRMS (EI): $\mathrm{m} / \mathrm{z}$ calcd for $\mathrm{C}_{25} \mathrm{H}_{24} \mathrm{~F}_{3} \mathrm{NO}_{2} \mathrm{~S}: 460.1553\left[M+\mathrm{H}^{+}\right]$; found: 460.1553 .

Ethyl $\quad\left(R_{S}, 3 S\right)-3-((t e r t-b u t y l$ sulfinyl)amino)-3-(8-((2e-methoxyphenyl)ethynyl)naphthalen-1yl)propanoate (10bb).

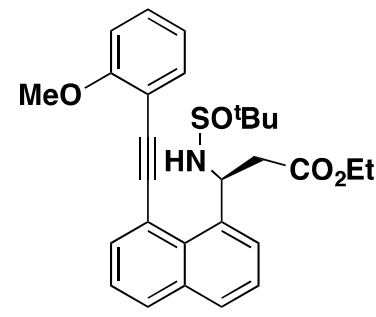

Compound 10bb was synthesized following the general procedure described above starting from $\mathbf{2 b}(300 \mathrm{mg}, 0.64 \mathrm{mmol}$ ) affording $\mathbf{1 0 b b}$ as a yellow-green oil (168 mg, $55 \%$ yield). $[\alpha]^{25}=-39.1\left(c=1.0\right.$ in $\left.^{2} \mathrm{CHCl}_{3}\right) .{ }^{1} \mathrm{H}$ NMR $\left(300 \mathrm{MHz}, \mathrm{CDCl}_{3}\right): 0.94(t, J$ $=7.3 \mathrm{~Hz}, 3 \mathrm{H}), 1.26(\mathrm{~s}, 9 \mathrm{H}), 3.05(\mathrm{dd}, J=15.7,6.5 \mathrm{~Hz}, 1 \mathrm{H}), 3.30(\mathrm{dd}, J=16.0,4.5 \mathrm{~Hz}, 1 \mathrm{H})$, $3.81(\mathrm{~m}, 2 \mathrm{H}), 3.98(\mathrm{~s}, 3 \mathrm{H}), 5.42(\mathrm{~d}, J=5.7 \mathrm{~Hz}, 1 \mathrm{H}), 6.92(\mathrm{dd}, J=8.2,0.9 \mathrm{~Hz}, 1 \mathrm{H}), 6.99(\mathrm{td}, J=$ 7.5, $1.0 \mathrm{~Hz}, 1 \mathrm{H}), 7.32$ (ddd, $J=9.2,7.4,1.7 \mathrm{~Hz}, 1 \mathrm{H}), 7.45$ (m, 2H), 7.66 (ddd, $J=7.6,1.7,0.4$ $\mathrm{Hz}, 1 \mathrm{H}), 7.77$ (br s, 1H), 7.80 (br s, 1H), 7.84 (dd, $J=8.1,7.4 \mathrm{~Hz}, 1 \mathrm{H}), 7.91$ (dd, $J=7.2,1.5$ $\mathrm{Hz}, 1 \mathrm{H}) .{ }^{13} \mathrm{C}$ NMR $\left(75.5 \mathrm{MHz}, \mathrm{CDCl}_{3}\right): 13.8\left(\mathrm{CH}_{3}\right), 22.8\left(3 \mathrm{xCH}_{3}\right), 42.6\left(\mathrm{CH}_{2}\right), 51.6(\mathrm{CH}), 55.8$ (C), $55.9\left(\mathrm{CH}_{3}\right), 60.5\left(\mathrm{CH}_{2}\right), 91.0(\mathrm{C}), 95.1(\mathrm{C}), 110.5(\mathrm{CH}), 112.5(\mathrm{C}), 118.6(\mathrm{C}), 120.5(\mathrm{CH})$, 
$124.8(\mathrm{CH}), 125.4(\mathrm{CH}), 126.3(\mathrm{CH}), 129.4(\mathrm{CH}), 129.5(\mathrm{CH}), 129.9(\mathrm{CH}), 130.4(\mathrm{CH}), 133.3$ (CH), $134.8(\mathrm{C}), 135.7(\mathrm{CH}), 138.7$ (C), 160.3 (C), 172.1 (C). HRMS (EI): m/z calcd for $\mathrm{C}_{28} \mathrm{H}_{31} \mathrm{NO}_{4} \mathrm{~S}: 478.2047\left[M+\mathrm{H}^{+}\right]$; found: 478.2043.

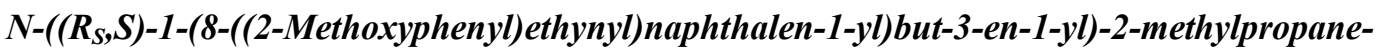
2-sulfinamide (10cb).

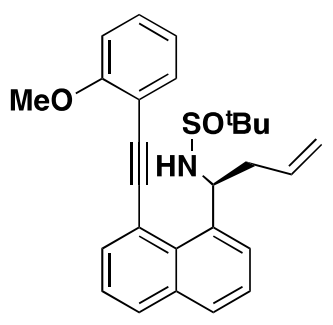

Compound 10cb was synthesized following the general procedure described above starting from $2 \mathbf{c}(300 \mathrm{mg}, 0.71 \mathrm{mmol})$ affording $10 \mathrm{cb}$ as a yellow-green oil (267 mg, $88 \%$ yield). $[\alpha]^{25}=-4.1\left(c=1.0\right.$ in $\left.\mathrm{CHCl}_{3}\right) .{ }^{1} \mathrm{H} \mathrm{NMR}\left(300 \mathrm{MHz}, \mathrm{CDCl}_{3}\right): 1.22(\mathrm{~s}$, 9H), 2.65 (m, 1H), 3.06 (m, 1H), $3.90(\mathrm{~d}, J=4.2 \mathrm{~Hz}, 1 \mathrm{H}), 3.96$ (s, 3H), $5.13(\mathrm{~m}, 2 \mathrm{H}), 5.75$ (dddd, $J=17.8,14.3,7.8,6.5 \mathrm{~Hz}, 1 \mathrm{H}), 6.93$ (dd, $J=8.4,1.0 \mathrm{~Hz}, 1 \mathrm{H}), 7.01$ (m, 2H), 7.33 (ddd, $J$ $=9.2,7.4,1.7 \mathrm{~Hz}, 1 \mathrm{H}), 7.45(\mathrm{~m}, 2 \mathrm{H}), 7.68(\mathrm{ddd}, J=7.5,1.7,0.4 \mathrm{~Hz}, 1 \mathrm{H}), 7.76(\mathrm{~m}, 2 \mathrm{H}), 7.84$ (ddd, $J=8.2,1.5,0.5 \mathrm{~Hz}, 1 \mathrm{H}), 7.91(\mathrm{~m}, 1 \mathrm{H}) .{ }^{13} \mathrm{C} \mathrm{NMR}\left(75.5 \mathrm{MHz}, \mathrm{CDCl}_{3}\right): 22.7\left(3 \mathrm{xCH}_{3}\right), 43.9$ $\left(\mathrm{CH}_{2}\right), 52.5\left(\mathrm{CH}_{3}\right), 55.8(\mathrm{C}), 91.0(\mathrm{C}), 95.3(\mathrm{C}), 110.6(\mathrm{CH}), 112.6(\mathrm{CH}), 118.8(\mathrm{C}), 119.1$ $\left(\mathrm{CH}_{2}\right), 120.7(\mathrm{C}), 124.7(\mathrm{CH}), 125.3(\mathrm{CH}), 126.2(\mathrm{CH}), 129.0(\mathrm{CH}), 129.8(\mathrm{CH}), 129.9(\mathrm{C})$, $130.3(\mathrm{CH}), 133.3(\mathrm{CH}), 134.6(\mathrm{CH}), 134.8(\mathrm{C}), 135.7(\mathrm{CH}), 139.9(\mathrm{C}), 160.1(\mathrm{C})$. HRMS (EI): $\mathrm{m} / \mathrm{z}$ calcd for $\mathrm{C}_{27} \mathrm{H}_{29} \mathrm{NO}_{2} \mathrm{~S}: 432.1996\left[M+\mathrm{H}^{+}\right]$; found: 432.1992.

Ethyl $\left(\boldsymbol{R}_{S}, 3 S\right)-3-((t e r t-b u t y l s u l f i n y l)$ amino)-3-(8-((4-

(trifluoromethyl)phenyl)ethynyl)naphthalen-1-yl)propanoate (10bc).

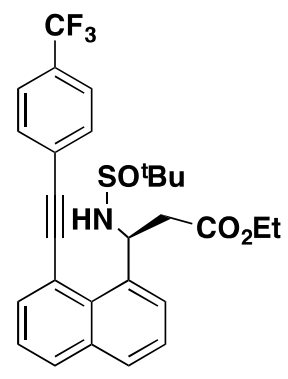

Compound 10bc was synthesized following the general procedure described above starting from $\mathbf{2 b}(300 \mathrm{mg}, 0.64 \mathrm{mmol})$ affording $\mathbf{1 0 b c}$ as a yellow-green oil (141 $\mathrm{mg}, 43$ \% yield). $[\alpha]_{\mathrm{D}}^{25}=+7.4\left(c=1.0\right.$ in $\left.\mathrm{CHCl}_{3}\right) .{ }^{1} \mathrm{H} \mathrm{NMR}\left(300 \mathrm{MHz}, \mathrm{CDCl}_{3}\right): 0.99$ (t, $J=7.1 \mathrm{~Hz}$, $3 \mathrm{H}), 1.28$ (s, 9H), 3.00 (dd, $J=15.7,7.7 \mathrm{~Hz}, 1 \mathrm{H}), 3.17$ (dd, $J=15.9,4.3 \mathrm{~Hz}, 1 \mathrm{H}), 3.85$ (m, 2H), $5.32(\mathrm{~d}, J=5.0 \mathrm{~Hz}, 1 \mathrm{H}), 7.48(\mathrm{~m}, 2 \mathrm{H}), 7.67(\mathrm{~m}, 2 \mathrm{H}), 7.83(\mathrm{~m}, 4 \mathrm{H}), 7.90(\mathrm{~m} 2 \mathrm{H}) .{ }^{19} \mathrm{~F}$ NMR $\left(282.4 \mathrm{MHz}, \mathrm{CDCl}_{3}\right) \delta-62.8\left(\mathrm{~s}, \mathrm{CF}_{3}\right) .{ }^{13} \mathrm{C} \mathrm{NMR}\left(75.5 \mathrm{MHz}, \mathrm{CDCl}_{3}\right): 13.8\left(\mathrm{CH}_{3}\right), 22.7\left(3 \mathrm{xCH}_{3}\right)$, 
$42.4\left(\mathrm{CH}_{2}\right), 51.1(\mathrm{CH}), 55.8(\mathrm{C}), 60.7\left(\mathrm{CH}_{2}\right), 92.9(\mathrm{C}), 93.5(\mathrm{C}), 117.4(\mathrm{C}), 124.8(\mathrm{CH}), 125.4$ $(\mathrm{CH}), 125.5(\mathrm{CH}), 125.6(\mathrm{CH}), 126.7(\mathrm{CH}), 127.0(\mathrm{CH}), 128.0\left(\mathrm{CF}_{3}\right), 128.2(\mathrm{C}), 129.6(\mathrm{CH})$, $129.8(\mathrm{C}), 131.3(\mathrm{CH}), 131.7(\mathrm{CH}), 132.2(\mathrm{C}), 134.9(\mathrm{C}), 136.0(\mathrm{CH}), 138.4(\mathrm{C}), 171.8(\mathrm{C})$. HRMS (EI): $\mathrm{m} / \mathrm{z}$ calcd for $\mathrm{C}_{28} \mathrm{H}_{28} \mathrm{~F}_{3} \mathrm{NO}_{3} \mathrm{~S}: 516.1701\left[M+\mathrm{H}^{+}\right]$; found: 516.1707 .

\section{2-Methyl-N-((R,S)-1-(8-((4-(trifluoromethyl)phenyl)ethynyl)naphthalen-1-yl)but-3-en-1-} yl)propane-2-sulfinamide (10cc).

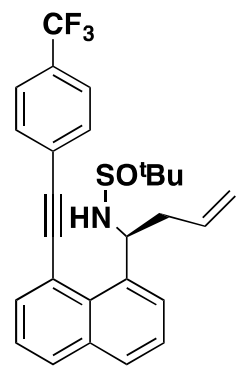

Compound 10ce was synthesized following the general procedure described above starting from $2 \mathbf{c}(300 \mathrm{mg}, 0.71 \mathrm{mmol})$ affording 10cc as a yellow-green oil (221 $\mathrm{mg}, 67$ $\%$ yield). $[\alpha]^{25}=-54.5\left(c=1.0\right.$ in $\left.\mathrm{CHCl}_{3}\right) .{ }^{1} \mathrm{H} \mathrm{NMR}\left(300 \mathrm{MHz}, \mathrm{CDCl}_{3}\right): 1.26(\mathrm{~s}, 9 \mathrm{H}), 2.58(\mathrm{~m}$, 1H), 2.95 (m, 1H), 3.92 (d, $J=3.4 \mathrm{~Hz}, 1 \mathrm{H}), 5.17$ (m, 2H), 5.68 (dddd, $J=17.9,14.3,7.8,6.4$ $\mathrm{Hz}, 1 \mathrm{H}), 7.04(\mathrm{dt}, J=8.0,4.0 \mathrm{~Hz}, 1 \mathrm{H}), 7.48(\mathrm{~m}, 2 \mathrm{H}), 7.68(\mathrm{~m}, 2 \mathrm{H}), 7.85(\mathrm{~m}, 6 \mathrm{H}) .{ }^{9} \mathrm{~F}$ NMR $\left(282.4 \mathrm{MHz}, \mathrm{CDCl}_{3}\right) \delta-62.8(\mathrm{~d}, J=7.2 \mathrm{~Hz}) .{ }^{13} \mathrm{C} \mathrm{NMR}\left(75.5 \mathrm{MHz}, \mathrm{CDCl}_{3}\right): 22.7\left(3 \mathrm{xCH}_{3}\right)$, $44.0\left(\mathrm{CH}_{2}\right), 52.1(\mathrm{CH}), 55.8(\mathrm{C}), 92.9(\mathrm{C}), 93.7(\mathrm{C}), 117.6(\mathrm{C}), 119.8\left(\mathrm{CH}_{2}\right), 122.2(\mathrm{CH}), 124.7$ $(\mathrm{CH}), 125.4(\mathrm{CH}), 125.5(\mathrm{CH}), 125.6(\mathrm{CH}), 125.8\left(\mathrm{q},{ }^{1} J_{\mathrm{CF}}=271.6 \mathrm{~Hz}, \mathrm{CF}_{3}\right), 126.5(\mathrm{CH}), 127.1$ (C), $129.1(\mathrm{CH}), 129.8(\mathrm{C}), 130.2(\mathrm{C}), 131.2(\mathrm{CH}), 131.7(\mathrm{CH}), 134.1(\mathrm{CH}), 134.8(\mathrm{C}), 135.9$ $(\mathrm{CH}), 139.9$ (C). HRMS (EI): $\mathrm{m} / \mathrm{z}$ calcd for $\mathrm{C}_{27} \mathrm{H}_{26} \mathrm{~F}_{3} \mathrm{NOS}$ : $470.1760\left[M+\mathrm{H}^{+}\right]$; found: 470.1769.

\section{General procedure for the removal of the tert-butanesulfinyl group/N-Boc-protection reaction to obtain carbamates 11aa-11cc.}
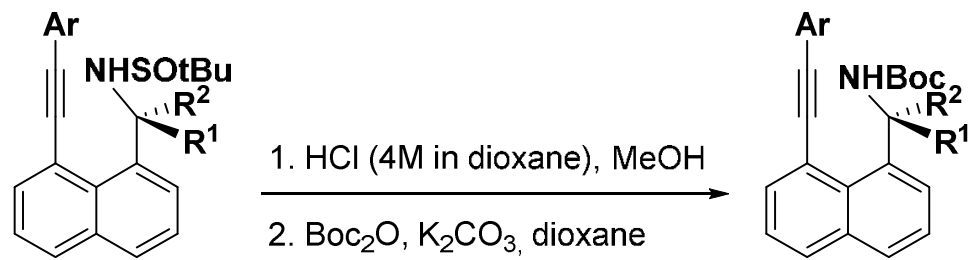

Hydrogen chloride (4.0 equiv, 4M solution in 1,4-dioxane) was added at room temperature to a solution of the corresponding sulfinyl amine 10aa-10cc (1.0 equiv) in $\mathrm{MeOH}$ (1 M). After 20 minutes the deprotection was complete and the solution was concentrated to dryness. The residue was redissolved in $\mathrm{CH}_{2} \mathrm{Cl}_{2}(0.1 \mathrm{M})$ and $2 \mathrm{M} \mathrm{NaOH}$ was added and 
extracted three times with dichloromethane and the combined organic layers were dried over anhydrous $\mathrm{Na}_{2} \mathrm{SO}_{4}$. Solvents were removed under reduced pressure and the resulting crude was dissolved in anhydrous 1,4-dioxane $(0.2 \mathrm{M})$ and treated with $\mathrm{K}_{2} \mathrm{CO}_{3}$ (1.1 equiv) and di-tertbutyl dicarbonate $\left(\mathrm{Boc}_{2} \mathrm{O}\right)$ (1.1 equiv) at room temperature until TLC revealed the disappearance of the free amine. The suspension was then filtered through a short pad of Celite washing with small portions of ethyl acetate. Solvents were removed under reduced pressure and the residue was purified by means of flash chromatography on silica gel (10:1 $n$ hexane:ethyl acetate) to afford carbamates 11aa-11cc.

tert-Butyl (S)-3-(8-(1-((tert-butoxycarbonyl)amino)-3-ethoxy-3-oxopropyl)naphthalen-1yl)propiolate (11ba).<smiles>CCOC(=O)CC(NC(=O)OCc1ccccc1)c1cccc2cccc(C#Cc3ccccc3)c12</smiles>

Compound $\mathbf{1 1 b a}$ was synthesized following the general procedure described above starting from 10ba (100 mg, $0.22 \mathrm{mmol}$ ) affording $11 \mathrm{ba}$ as a yellow oil (67 $\mathrm{mg}, 68 \%$ yield). $[\alpha]^{25}=+56.4\left(c=1.0\right.$ in $\left.\mathrm{CHCl}_{3}\right) .{ }^{1} \mathrm{H} \mathrm{NMR}\left(300 \mathrm{MHz}, \mathrm{CDCl}_{3}\right): 0.97(t, J=7.2 \mathrm{~Hz}, 3 \mathrm{H})$ 1.45 (s, 9H), 3.05 (m, 2H), 3.88 (m, 2H), 6.19 (br s, 1H), 7.42 (m, 6H), 7.68 (m, 2H), 7.77 (dd, $J$ $=4.8,1.4 \mathrm{~Hz}, 1 \mathrm{H}), 7.86(\mathrm{~m}, 2 \mathrm{H}) .{ }^{13} \mathrm{C} \mathrm{NMR}\left(75.5 \mathrm{MHz}, \mathrm{CDCl}_{3}\right): 13.8\left(3 \mathrm{xCH}_{3}\right), 21.5\left(\mathrm{CH}_{3}\right), 28.3$ (CH), $41.2\left(\mathrm{CH}_{2}\right), 60.4\left(\mathrm{CH}_{2}\right), 79.3(\mathrm{C}), 91.3(\mathrm{C}), 93.9(\mathrm{C}), 118.3(\mathrm{C}), 123.5(\mathrm{C}), 124.7(2 \mathrm{xCH})$, $124.9(\mathrm{C}), 125.5(\mathrm{CH}), 128.3(3 \times \mathrm{xH}), 129.1(\mathrm{CH}), 129.5(\mathrm{C}), 130.5(\mathrm{CH}), 131.4(\mathrm{CH}), 134.9$ (C), 135.3 (2xCH), 154.9 (C), 171.5 (C). HRMS (EI): m/z calcd for $\mathrm{C}_{27} \mathrm{H}_{29} \mathrm{NO}_{3} \mathrm{~S}: 466.1989$ [M $+\mathrm{Na}^{+}$; found: 466.2009 .

tert-Butyl (S)-(1-(8-(phenylethynyl)naphthalen-1-yl)but-3-en-1-yl)carbamate (11ca).<smiles>C=CCC(NC(=O)OCc1ccccc1)c1cccc2ccccc12</smiles>

Compound 11ca was synthesized following the general procedure described above starting from 10ca (100 mg, $0.25 \mathrm{mmol}$ ) affording 11 ca as a yellow oil (69 $\mathrm{mg}, 70 \%$ yield). $[\alpha]^{25}=-23.4\left(c=1.0\right.$ in $\left.\mathrm{CHCl}_{3}\right) .{ }^{1} \mathrm{H} \mathrm{NMR}\left(300 \mathrm{MHz}, \mathrm{CDCl}_{3}\right): 1.44$ (s, 9H), 2.55 (br s, 1H), 2.80 (br s, 1H), 5.07 (m, 2H), 5.67 (ddd, $J=17.1,10.1,7.1 \mathrm{~Hz}, 1 \mathrm{H}), 7.15$ (dt, $J=7.4,4.5$ $\mathrm{Hz}, 1 \mathrm{H}), 7.43(\mathrm{~m}, 5 \mathrm{H}), 7.62(\mathrm{~m}, 3 \mathrm{H}), 7.77(\mathrm{dd}, J=8.6,1.6 \mathrm{~Hz}, 1 \mathrm{H}), 7.86(\mathrm{~m}, 2 \mathrm{H}) .{ }^{13} \mathrm{C} \mathrm{NMR}$ (75.5 MHz, $\left.\mathrm{CDCl}_{3}\right): 28.4\left(3 \mathrm{xCH}_{3}\right), 42.3\left(\mathrm{CH}_{2}\right), 49.9(\mathrm{CH}), 79.4(\mathrm{C}), 91.7(\mathrm{C}), 93.8(\mathrm{C}), 118.5$ $\left(\mathrm{CH}_{2}\right), 123.6(\mathrm{C}), 124.2(\mathrm{CH}), 124.6(3 \times \mathrm{CH}), 125.5(\mathrm{CH}), 128.3(\mathrm{CH}), 128.4(2 \times \mathrm{CH}), 128.7(\mathrm{C})$, 
129.7 (C), 130.5 (2xCH), $131.4(\mathrm{CH}), 133.9$ (C), 134.9 (C), $135.2(\mathrm{CH}), 155.1(\mathrm{C})$. HRMS (EI): $\mathrm{m} / \mathrm{z}$ calcd for $\mathrm{C}_{27} \mathrm{H}_{27} \mathrm{NO}_{2}: 398.2116\left[M+\mathrm{H}^{+}\right]$; found: 398.2115 .

tert-Butyl (S)-(2,2,2-trifluoro-1-(8-((2-methoxyphenyl)ethynyl)naphthalen-1yl)ethyl)carbamate (11ab).<smiles>COc1ccccc1C#Cc1cccc2cccc(NC(=O)OC(F)(F)F)c12</smiles>

Compound 11ab was synthesized following the general procedure described above starting from 10ab (100 $\mathrm{mg}, 0.22 \mathrm{mmol})$ affording 11ab as a yellow oil (37 $\mathrm{mg}, 37 \%$ yield). $[\alpha]_{\mathrm{D}}^{25}=-41.1\left(c=1.0\right.$ in $\left.\mathrm{CHCl}_{3}\right) .{ }^{1} \mathrm{H} \mathrm{NMR}\left(300 \mathrm{MHz}, \mathrm{CDCl}_{3}\right): 1.26(\mathrm{~s}, 9 \mathrm{H})$, $3.93(\mathrm{~s}, 3 \mathrm{H}), 5.54$ (br s, 1H), $6.96(\mathrm{~m}, 2 \mathrm{H}), 7.33(\mathrm{~m}, 1 \mathrm{H}), 7.47$ (m, 1H), 7.54 (d, J= 7.6 Hz, 1H), $7.65(\mathrm{~m}, 1 \mathrm{H}), 7.87(\mathrm{~m}, 2 \mathrm{H}), 7.95(\mathrm{dd}, J=7.2,1.4 \mathrm{~Hz}, 1 \mathrm{H}), 8.37(\mathrm{~m}, 1 \mathrm{H}) .{ }^{19} \mathrm{~F}$ NMR $(282.4 \mathrm{MHz}$, $\left.\mathrm{CDCl}_{3}\right) \delta-73.7\left(\mathrm{~d}, J=7.2 \mathrm{~Hz}, \mathrm{CF}_{3}\right) .{ }^{13} \mathrm{C} \mathrm{NMR}\left(75.5 \mathrm{MHz}, \mathrm{CDCl}_{3}\right): 28.1\left(3 \mathrm{xCH}_{3}\right), 29.7(\mathrm{CH})$, $55.6\left(\mathrm{CH}_{3}\right), 80.5(\mathrm{C}), 92.7(\mathrm{C}), 94.4(\mathrm{C}), 110.5(2 \mathrm{xCH}), 112.6(\mathrm{C}), 119.2(\mathrm{C}), 120.4(2 \mathrm{xCH})$, $125.1(\mathrm{CH}), 125.3(\mathrm{CH}), 126.9\left(\mathrm{CF}_{3}\right), 129.9(\mathrm{CH}), 130.2(\mathrm{CH}), 130.5(\mathrm{C}), 130.8(\mathrm{CH}), 133.3$ (C), $134.6(\mathrm{CH}), 136.2(\mathrm{C}), 154.3(\mathrm{C}), 160.3(\mathrm{C})$. HRMS (EI): $\mathrm{m} / \mathrm{z}$ calcd for $\mathrm{C}_{26} \mathrm{H}_{24} \mathrm{~F}_{3} \mathrm{NO}_{3}$ : $456.1792\left[M+\mathrm{H}^{+}\right]$; found: 456.1781 .

Ethyl (S)-3-((tert-butoxycarbonyl)amino)-3-(8-((2-methoxyphenyl)ethynyl)naphthalen-1yl)propanoate (11bb).<smiles>CCOC(=O)CNC(C#Cc1cccc2cccc(C(=O)OCC)c12)c1ccccc1OC</smiles>

Compound $11 \mathbf{b b}$ as synthesized following the general procedure described above starting from $10 \mathbf{b b}(100 \mathrm{mg}, 0.21 \mathrm{mmol})$ affording $\mathbf{1 1 b b}$ as a yellow oil (63 $\mathrm{mg}, 71 \%$ yield). $[\alpha]^{25}=+34.4\left(c=1.0\right.$ in $\left.\mathrm{CHCl}_{3}\right) .{ }^{1} \mathrm{H}$ NMR $\left(300 \mathrm{MHz}, \mathrm{CDCl}_{3}\right): 0.95(t, J=7.1$ Hz, 3H), 1.43 (s, 9H), 3.10 (m, 2H), 3.86 (q, $J=7.1 \mathrm{~Hz}, 2 \mathrm{H}), 3.93$ (s, 3H), 6.54 (br s, 1H), 6.96 (m, 2H), $7.32(\mathrm{~m}, 1 \mathrm{H}), 7.44(\mathrm{~m}, 2 \mathrm{H}), 7.61(\mathrm{~m}, 1 \mathrm{H}), 7.70(\mathrm{dd}, J=7.6,1.7 \mathrm{~Hz}, 1 \mathrm{H}), 7.76(\mathrm{dd}, J=$ $8.3,1.5 \mathrm{~Hz}, 1 \mathrm{H}), 7.83(\mathrm{dd}, J=8.2,1.6 \mathrm{~Hz}, 1 \mathrm{H}), 7.89(\mathrm{dd}, J=7.3,1.7 \mathrm{~Hz}, 1 \mathrm{H}) .{ }^{13} \mathrm{C}$ NMR $(75.5$ 
$\left.\mathrm{MHz}, \mathrm{CDCl}_{3}\right): 13.8(3 \mathrm{xCH}), 28.4\left(\mathrm{CH}_{3}\right), 40.8\left(\mathrm{CH}_{2}\right), 47.7(\mathrm{CH}), 55.6(\mathrm{CH}), 60.3\left(\mathrm{CH}_{2}\right), 79.1$ (C), $90.4(\mathrm{C}), 95.3(\mathrm{C}), 110.5(\mathrm{CH}), 112.9(\mathrm{C}), 118.7(\mathrm{C}), 120.5(\mathrm{CH}), 124.6(\mathrm{CH}), 124.9(\mathrm{CH})$, $125.5(\mathrm{CH}), 129.0(\mathrm{CH}), 129.7(\mathrm{CH}), 130.4(\mathrm{CH}), 133.3(\mathrm{CH}), 134.9(\mathrm{C}), 135.4(\mathrm{CH}), 138.8$ (C), 154.8 (C), $160.2(\mathrm{C}), 172.1$ (C). HRMS (EI): m/z calcd for $\mathrm{C}_{29} \mathrm{H}_{31} \mathrm{NO}_{5}: 474.2283\left[M^{+}+\mathrm{H}\right]$; found: 474.2275 .

tert-Butyl (1-(8-((2-methoxyphenyl)ethynyl)naphthalen-1-yl)but-3-en-1-yl)carbamate (11cb).<smiles>C=CCC(NC(=O)OCc1ccccc1OC)c1cccc2cccc(C#CC(=O)OC)c12</smiles>

Compound 11cb was synthesized following the general procedure described above starting from 10cb (100 $\mathrm{mg}, 0.23 \mathrm{mmol})$ affording $11 \mathrm{cb}$ as a yellow oil $(67 \mathrm{mg}$, $68 \%$ yield). $[\alpha]^{25}=+40.6\left(c=1.0\right.$ in $\left._{\mathrm{D}} \mathrm{CHCl}_{3}\right) .{ }^{1} \mathrm{H} \mathrm{NMR}\left(300 \mathrm{MHz}, \mathrm{CDCl}_{3}\right): 1.53(\mathrm{~s}, 9 \mathrm{H}), 3.92$ (s, 3H), 5.07 (m, 2H), $5.68(\mathrm{dd}, J=17.2,6.9 \mathrm{~Hz}, 1 \mathrm{H}), 6.96(\mathrm{~m}, 2 \mathrm{H}), 7.11(\mathrm{~s}, 1 \mathrm{H}), 7.33(\mathrm{~m}, 1 \mathrm{H})$, $7.41(\mathrm{~m}, 1 \mathrm{H}), 7.47(\mathrm{~d}, J=7.6 \mathrm{~Hz}, 1 \mathrm{H}), 7.61(\mathrm{~d}, J=6.5 \mathrm{~Hz}, 1 \mathrm{H}), 7.75(\mathrm{dd}, J=7.9,0.9 \mathrm{~Hz}, 1 \mathrm{H})$, $7.84(\mathrm{~d}, J=8.1 \mathrm{~Hz}, 1 \mathrm{H}), 7.88(\mathrm{dd}, J=7.2,1.4 \mathrm{~Hz}, 1 \mathrm{H}) .{ }^{13} \mathrm{C} \mathrm{NMR}\left(75.5 \mathrm{MHz}, \mathrm{CDCl}_{3}\right): 27.6$ (CH3), $28.6\left(\mathrm{CH}_{3}\right), 42.0\left(\mathrm{CH}_{2}\right), 50.0(\mathrm{CH}), 55.7\left(\mathrm{CH}_{3}\right), 79.2(\mathrm{C}), 90.6(\mathrm{C}), 95.8(\mathrm{C}), 110.7$ $(2 \times C H), 120.7(2 \times C H), 124.5(C), 124.7(\mathrm{CH}), 125.6(2 \times C H), 128.7(\mathrm{C}), 129.9(\mathrm{CH}), 130.5$ (2xCH), 133.5 (C), 134.8 (C), 135.5 (C), 160.3 (C). HRMS (EI): m/z calcd for $\mathrm{C}_{28} \mathrm{H}_{29} \mathrm{NO}_{3}$ : $428.2221\left[M+\mathrm{H}^{+}\right]$; found: 428.2220 .

\section{Ethyl (S)-3-((tert-butoxycarbonyl)amino)-3-(8-((4-(trifluoromethyl)phenyl)ethynyl)} naphthalen-1-yl)propanoate (11bc).

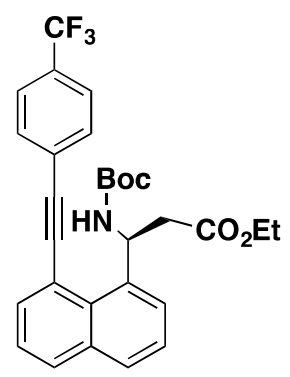

Compound 11bc was synthesized following the general procedure described above starting from 10bc (100 mg, $0.19 \mathrm{mmol}$ ) affording 11bc as a yellow oil (57 $\mathrm{mg} 57 \%$ yield). $[\alpha]^{25}=+46.8\left(c=1.0\right.$ in $\left.\mathrm{CHCl}_{3}\right) .{ }^{1} \mathrm{H} \mathrm{NMR}\left(300 \mathrm{MHz}, \mathrm{CDCl}_{3}\right): 0.97(t, J=7.1 \mathrm{~Hz}, 3 \mathrm{H})$, 1.53 (s, 9H), 2.99 (d, $J=6.3 \mathrm{~Hz}, 2 \mathrm{H}), 3.88$ (q, $J=7.1 \mathrm{~Hz}, 2 \mathrm{H}), 6.04$ (br s, 1H), 7.47 (m, 2H), $7.63(\mathrm{~m}, 2 \mathrm{H}), 7.69$ (d, $J=7.4 \mathrm{~Hz}, 1 \mathrm{H}), 7.80(\mathrm{~m}, 3 \mathrm{H}), 7.88$ (br s, 1H), 7.90 (br s, $1 \mathrm{H}) .{ }^{19} \mathrm{~F} \mathrm{NMR}$ 
$\left(282.4 \mathrm{MHz}, \mathrm{CDCl}_{3}\right) \delta-62.8\left(\mathrm{~s}, \mathrm{CF}_{3}\right) .{ }^{13} \mathrm{C} \mathrm{NMR}\left(75.5 \mathrm{MHz}, \mathrm{CDCl}_{3}\right): 14.0\left(3 \mathrm{xCH}_{3}\right), 27.6\left(\mathrm{CH}_{3}\right)$, $28.5(\mathrm{CH}), 41.4\left(\mathrm{CH}_{2}\right), 60.6\left(\mathrm{CH}_{2}\right), 78.0(\mathrm{C}), 85.3(\mathrm{C}), 93.7(\mathrm{C}), 124.9(\mathrm{CH}), 125.4(\mathrm{CH}), 125.9$ $(\mathrm{CH}), 127.5(\mathrm{C}), 129.5(\mathrm{CH}), 131.4(\mathrm{CH}), 131.9(\mathrm{CH}), 135.0(\mathrm{C}), 135.9(\mathrm{CH}), 138.8(\mathrm{C}), 155.2$ (C), 171.5 (C). HRMS (EI): $\mathrm{m} / \mathrm{z}$ calcd for $\mathrm{C}_{29} \mathrm{H}_{28} \mathrm{NO}_{4} \mathrm{~F}_{3}: 512.2043\left[M+\mathrm{H}^{+}\right]$; found: 512.2031

tert-Butyl (1-(8-((4-(trifluoromethyl)phenyl)ethynyl)naphthalen-1-yl)but-3-en-1-yl) carbamate (11cc).

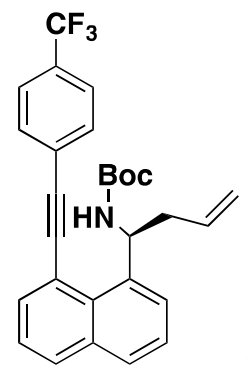

Compound 11cc was synthesized following the general procedure described above starting from 10cc (100 mg, $0.21 \mathrm{mmol})$ affording 11cc as a yellow oil (59 $\mathrm{mg}, 60 \%$ yield). $[\alpha]_{D}^{25}=+32.9\left(c=1.0\right.$ in $\left.\mathrm{CHCl}_{3}\right) .{ }^{1} \mathrm{H}$ NMR $\left(300 \mathrm{MHz}, \mathrm{CDCl}_{3}\right): 1.26(\mathrm{~s}, 9 \mathrm{H}), 2.51(\mathrm{~m}$, 1H), 2.77 (m, 1H), 5.06 (m, 3H), 5.63 (dt, $J=24.3,7.2 \mathrm{~Hz}, 1 \mathrm{H}), 7.13$ (br s, 1H), 7.48 (m, 1H), $7.64(\mathrm{~m}, 2 \mathrm{H}), 7.78(\mathrm{dd}, J=7.9,1.3 \mathrm{~Hz}, 2 \mathrm{H}), 7.88(\mathrm{dt}, J=8.3,3.1 \mathrm{~Hz}, 2 \mathrm{H}) .{ }^{19} \mathrm{~F}$ NMR $(282.4$ $\left.\mathrm{MHz}, \mathrm{CDCl}_{3}\right) \delta-62.5\left(\mathrm{~s}, \mathrm{CF}_{3}\right)$. HRMS (EI): $\mathrm{m} / \mathrm{z}$ calcd for $\mathrm{C}_{28} \mathrm{H}_{26} \mathrm{~F}_{3} \mathrm{NO}_{2}: 466.1998\left[M+\mathrm{H}^{+}\right]$; found: 466.1990 .

\section{General procedure for gold(I)-catalyzed intramolecular hydroamination reaction.}

To a solution of the corresponding alkynyl carbamate 11 (1 equiv) and ethanol (5 equiv) in DCE $(0.1 \mathrm{M})$ was added AuSPhosNTf2 (0.05 equiv) in DCE at $40^{\circ} \mathrm{C}$ drop-wise (see optimization table S1 below, page S29). The resulting solution was stirred at $40^{\circ} \mathrm{C}$ until TLC revealed the disappearance of the starting material. The solvent was then removed under reduced pressure and the crude mixture was purified by flash chromatography on silica gel using mixtures of $n$-hexane:ethyl acetate (3:1) as eluents to afford compounds 12aa-12cc.

tert-Butyl (S,E)-1-benzylidene-3-(2-ethoxy-2-oxoethyl)-1H-benzo[de]isoquinoline-2(3H)carboxylate (12ba). 


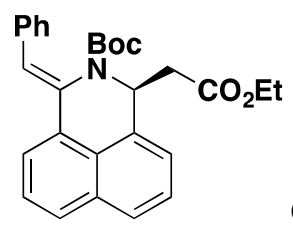

Compound 12ba was synthesized following the general procedure described above starting from 11ba (50 mg, $0.11 \mathrm{mmol}$ ) affording 12ba as a colorless oil (38 $\mathrm{mg}, 76 \%$ yield). $[\alpha]^{25}=+48.3\left(c=1.0\right.$ in $\left.\mathrm{CHCl}_{3}\right) .{ }^{1} \mathrm{H}$ NMR $\left(300 \mathrm{MHz}, \mathrm{CDCl}_{3}\right): 1.10(\mathrm{~s}, 9 \mathrm{H}), 1.11(\mathrm{t}, J=$ $7.2 \mathrm{~Hz}, 3 \mathrm{H}), 2.66$ (dd, $J=15.3,6.6 \mathrm{~Hz}, 1 \mathrm{H}), 2.98(\mathrm{dd}, J=15.6,7.8 \mathrm{~Hz}, 1 \mathrm{H}), 4.04(\mathrm{~m}, 2 \mathrm{H}), 6.30$ (br s, 1H), $7.20(\mathrm{~m}, 1 \mathrm{H}), 7.37$ (m, 5H), 7.53 (dd, $J=8.2,7.3 \mathrm{~Hz}, 1 \mathrm{H}), 7.65$ (m, 2H), 7.76 (dd, $J$ $=7.9,1.8 \mathrm{~Hz}, 1 \mathrm{H}), 7.82(\mathrm{dd}, J=8.4,1.0 \mathrm{~Hz}, 1 \mathrm{H}), 7.90(\mathrm{dd}, J=7.3,1.0 \mathrm{~Hz}, 1 \mathrm{H}) .{ }^{13} \mathrm{C} \mathrm{NMR}(75.5$ $\left.\mathrm{MHz}, \mathrm{CDCl}_{3}\right)$ : $13.1\left(\mathrm{CH}_{3}\right), 27.9\left(3 \mathrm{xCH}_{3}\right), 41.3\left(\mathrm{CH}_{2}\right), 53.5(\mathrm{CH}), 60.6\left(\mathrm{CH}_{2}\right), 80.9(\mathrm{C}), 104.4$ (C), $120.7(\mathrm{CH}), 123.1(\mathrm{CH}), 125.3(\mathrm{CH}), 125.7(\mathrm{CH}), 125.8(\mathrm{CH}), 126.2(\mathrm{C}), 127.2(\mathrm{CH})$, $127.8(\mathrm{CH}), 128.1(\mathrm{CH}), 128.3(2 \mathrm{xCH}), 129.0(\mathrm{CH}), 131.4(\mathrm{C}), 132.3(\mathrm{C}), 133.6(\mathrm{C}), 134.2(\mathrm{C})$, 135.6 (C). HRMS (EI): $\mathrm{m} / \mathrm{z}$ calcd for $\mathrm{C}_{28} \mathrm{H}_{29} \mathrm{NO}_{4}: 444.2169\left[M+\mathrm{H}^{+}\right]$; found: 444.2154

HPLC (Chiralpak IC, 92:8 hexane $\left./ \mathrm{PPrOH}, 1 \mathrm{~mL} \mathrm{~min}^{-1}, 240 \mathrm{~nm}\right) t \mathrm{R}($ major $)=11.56 \mathrm{~min}$, $t \mathrm{R}($ minor $)=23.36 \mathrm{~min}$.

tert-Butyl

(S)-(1-(8-((4-(trifluoromethyl)phenyl)ethynyl)naphthalen-1-yl)but-3-en-1-

yl)carbamate (12ca)

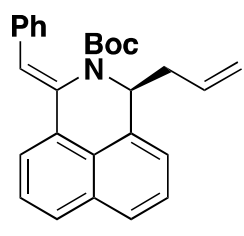

Compound 12ca was synthesized following the general procedure described above starting from 11ca (50 mg, $0.13 \mathrm{mmol}$ ) affording 12ca as a colorless oil (42 $\mathrm{mg}, 84 \%$ yield). $[\alpha]_{\mathrm{D}}^{25}=+82.2\left(c=1.0 \mathrm{in} \mathrm{CHCl}_{3}\right) .{ }^{1} \mathrm{H} \mathrm{NMR}\left(300 \mathrm{MHz}, \mathrm{CDCl}_{3}\right): 1.08(\mathrm{~s}, 9 \mathrm{H}), 2.51(\mathrm{dd}, J$ $=14.2,7.2 \mathrm{~Hz}, 1 \mathrm{H}), 2.78(\mathrm{~m}, 1 \mathrm{H}), 4.96(\mathrm{~m}, 2 \mathrm{H}), 5.83(\mathrm{~m}, 2 \mathrm{H}), 7.27(\mathrm{~m}, 3 \mathrm{H}), 7.40(\mathrm{~m}, 3 \mathrm{H}), 7.53$ $(\mathrm{m}, 1 \mathrm{H}), 7.73(\mathrm{~m}, 3 \mathrm{H}), 7.82(\mathrm{dd}, J=8.3,1.0 \mathrm{~Hz}, 1 \mathrm{H}), 7.92(\mathrm{dd}, J=7.4,1.0 \mathrm{~Hz}, 1 \mathrm{H}) .{ }^{13} \mathrm{C} \mathrm{NMR}$ (75.5 MHz, $\left.\mathrm{CDCl}_{3}\right): 27.9\left(3 \times \mathrm{CH}_{3}\right), 40.8\left(\mathrm{CH}_{2}\right), 57.0(\mathrm{CH}), 80.7(\mathrm{C}), 117.8\left(\mathrm{CH}_{2}\right), 120.4(\mathrm{CH})$, $123.7(\mathrm{CH}), 123.9(\mathrm{CH}), 125.4(\mathrm{CH}), 125.6(\mathrm{CH}), 126.4(\mathrm{C}), 126.7(\mathrm{CH}), 127.6(2 \times \mathrm{CH}), 128.0$ $(\mathrm{CH}), 128.3(2 \times C H), 129.1(\mathrm{CH}), 131.6(\mathrm{C}), 132.6(\mathrm{C}), 133.5(\mathrm{C}), 134.5(\mathrm{C}), 134.8(\mathrm{CH}), 136.1$ (C). HRMS (EI): $\mathrm{m} / \mathrm{z}$ calcd for $\mathrm{C}_{27} \mathrm{H}_{27} \mathrm{NO}_{2}: 420.1934\left[M+\mathrm{Na}^{+}\right]$; found: 420.1936 .

HPLC (Chiralpak ODH, 98:2 hexane $\left./ \mathrm{PrOH}, 1 \mathrm{~mL} \mathrm{~min}^{-1}, 240 \mathrm{~nm}\right) t \mathrm{R}($ major $)=7.88 \mathrm{~min}$, $t \mathrm{R}($ minor $)=9.07 \mathrm{~min}$. 
tert-Butyl (S,E)-1-(2-methoxybenzylidene)-3-(trifluoromethyl)-1H-benzo[de]isoquinoline2(3H)-carboxylate (12ab).<smiles>COc1ccccc1/C=C1\c2cccc3cccc(c23)[C@H](C(F)(F)F)N1C(F)(F)F</smiles>

Compound 12ab was synthesized following the general procedure described above starting from 11ab $(50 \mathrm{mg}, 0.11 \mathrm{mmol})$ affording 12ab as a colorless oil (48 mg, $96 \%$ yield). $[\alpha]^{25}=-8.7\left(c=1.0\right.$ in $\left.\mathrm{CHCl}_{3}\right) .{ }^{1} \mathrm{H} \mathrm{NMR}\left(300 \mathrm{MHz}, \mathrm{CDCl}_{3}\right): 1.03(\mathrm{~s}, 9 \mathrm{H})$, $3.93(\mathrm{~s}, 3 \mathrm{H}), 6.35(\mathrm{~d}, J=8.6 \mathrm{~Hz}, 1 \mathrm{H}), 6.96(\mathrm{~m}, 2 \mathrm{H}), 7.29(\mathrm{~m}, 1 \mathrm{H}), 7.53(\mathrm{~m}, 2 \mathrm{H}), 7.58(\mathrm{~m}, 1 \mathrm{H})$, 7.68 (br s, 1H), 7.84 (dd, $J=8.4,1.1 \mathrm{~Hz}, 1 \mathrm{H}), 7.89$ (m, 1H), $7.94(\mathrm{~m}, 1 \mathrm{H}), 8.08$ (dd, $J=7.3,1.2$ $\mathrm{Hz}, 1 \mathrm{H}) .{ }^{19} \mathrm{~F}$ NMR $\left(282.4 \mathrm{MHz}, \mathrm{CDCl}_{3}\right) \delta-71.8\left(\mathrm{~s}, \mathrm{CF}_{3}\right) \cdot{ }^{13} \mathrm{C} \mathrm{NMR}\left(75.5 \mathrm{MHz}, \mathrm{CDCl}_{3}\right): 27.5$ $\left(3 \times \mathrm{xH}_{3}\right), 29.7(\mathrm{CH}), 55.6\left(\mathrm{CH}_{3}\right), 81.9(\mathrm{C}), 110.5(2 \times \mathrm{CH}), 117.3(\mathrm{C}), 120.8(\mathrm{C}), 121.4(\mathrm{CH})$, $124.5\left(\mathrm{CF}_{3}\right), 125.2(\mathrm{C}), 125.4(\mathrm{C}), 126.2(2 \mathrm{xCH}), 126.7(\mathrm{C}), 127.7(2 \mathrm{xCH}), 128.7(\mathrm{CH}), 128.8$ $(\mathrm{CH}), 130.2(\mathrm{C}), 130.6(\mathrm{CH}), 133.3(\mathrm{C}), 157.0(\mathrm{C})$. HRMS (EI): m/z calcd for $\mathrm{C}_{26} \mathrm{H}_{24} \mathrm{~F}_{3} \mathrm{NO}_{3}$ : $456.1785\left[M+\mathrm{H}^{+}\right]$; found: 456.1781

tert-Butyl (S,E)-1-(2-ethoxy-2-oxoethyl)-3-(2-methoxybenzylidene)-1Hbenzo[de]isoquinoline-2(3H)-carboxylate (12bb).<smiles></smiles>

Compound 12bb was synthesized following the general procedure described above starting from $\mathbf{1 1 b b}(50 \mathrm{mg}, 0.11 \mathrm{mmol})$ affording $\mathbf{1 2 b b}$ as a colorless oil (42 mg, $83 \%$ yield). $[\alpha]^{25}=-27\left(c=1.0\right.$ in $\left.\mathrm{CHCl}_{3}\right) .{ }^{1} \mathrm{H}$ NMR $\left(300 \mathrm{MHz}, \mathrm{CDCl}_{3}\right): 1.12(\mathrm{~s}, 9 \mathrm{H})$, $1.26(\mathrm{t}, J=5.1 \mathrm{~Hz}, 3 \mathrm{H}), 2.66(\mathrm{dd}, J=15.6,7.5 \mathrm{~Hz}, 1 \mathrm{H}), 2.99(\mathrm{dd}, J=15.9,7.7 \mathrm{~Hz}, 1 \mathrm{H}), 3.91(\mathrm{~s}$, 3H), $4.06(\mathrm{~m}, 2 \mathrm{H}), 6.27$ (br s, 1H), $6.93(\mathrm{~m}, 2 \mathrm{H}), 7.24(\mathrm{~m}, 1 \mathrm{H}), 7.40(\mathrm{~m}, 2 \mathrm{H}), 7.53$ (dd, $J=8.2$, $7.4 \mathrm{~Hz}, 1 \mathrm{H}), 7.78(\mathrm{~m}, 4 \mathrm{H}), 7.95(\mathrm{dd}, J=7.4,1.1 \mathrm{~Hz}, 1 \mathrm{H}) .{ }^{13} \mathrm{C} \mathrm{NMR}\left(75.5 \mathrm{MHz}, \mathrm{CDCl}_{3}\right): 14.0$ $\left(\mathrm{CH}_{3}\right), 27.9\left(3 \times \mathrm{xCH}_{3}\right), 41.3\left(\mathrm{CH}_{2}\right), 55.6(\mathrm{CH}), 60.6\left(\mathrm{CH}_{2}\right), 80.7(\mathrm{C}), 110.6(2 \times \mathrm{CH}), 120.4(\mathrm{CH})$, $125.6(\mathrm{C}), 125.9(\mathrm{CH}), 126.3(\mathrm{C}), 127.1(\mathrm{CH}), 127.8(2 \times \mathrm{xH}), 128.2(\mathrm{CH}), 128.9(\mathrm{CH}), 131.8$ (C), 133.6 (C), 134.3 (C), 153.7 (C), 157.2 (C), 170.5 (C). HRMS (EI): m/z calcd for $\mathrm{C}_{29} \mathrm{H}_{31} \mathrm{NO}_{5}$ : $474.2286\left[M+\mathrm{H}^{+}\right]$; found: 474.2275 . 
HPLC (Chiralpak AD, 95:5 hexane $\left./ \mathrm{PrOH}, 1 \mathrm{~mL} \mathrm{~min}^{-1}, 240 \mathrm{~nm}\right) t \mathrm{R}$ (major) $=33.05 \mathrm{~min}$, $t \mathrm{R}($ minor $)=61,8 \mathrm{~min}$.

tert-Butyl

(S,E)-1-allyl-3-(2-methoxybenzylidene)-1H-benzo[de]isoquinoline-2(3H)carboxylate (12cb).<smiles></smiles>

Compound 12cb was synthesized following the general procedure described above starting from 11cb $(50 \mathrm{mg}, 0.12 \mathrm{mmol})$ affording $12 \mathrm{cb}$ as a colorless oil (45 mg, $90 \%$ yield). $[\alpha]^{25}=+334\left(c=1.0\right.$ in $\left.\mathrm{CHCl}_{3}\right) .{ }^{1} \mathrm{H}$ NMR $\left(300 \mathrm{MHz}, \mathrm{CDCl}_{3}\right): 1.06(\mathrm{~s}, 9 \mathrm{H})$, $2.52(\mathrm{~m}, 1 \mathrm{H}), 2.78(\mathrm{br} \mathrm{s}, 1 \mathrm{H}), 3.91(\mathrm{~s}, 3 \mathrm{H}), 4.96(\mathrm{~m}, 2 \mathrm{H}), 5.78$ (br s, 2H), $6.93(\mathrm{~m}, 2 \mathrm{H}), 7.23(\mathrm{~m}$, 2H), $7.42(\mathrm{dd}, J=8.3,7.1 \mathrm{~Hz}, 1 \mathrm{H}), 7.52$ (dd, $J=8.3,7.4 \mathrm{~Hz}, 1 \mathrm{H}), 7.67$ (br s, $1 \mathrm{H}), 7.74$ (dd, $J=$ 8.3, $1.2 \mathrm{~Hz}, 1 \mathrm{H}), 7.80(\mathrm{dd}, J=8.3,1.0 \mathrm{~Hz}, 1 \mathrm{H}), 7.97$ (m, 2H). ${ }^{13} \mathrm{C} \mathrm{NMR}\left(75.5 \mathrm{MHz}, \mathrm{CDCl}_{3}\right)$ : $28.0\left(3 \mathrm{xCH}_{3}\right), 40.8\left(\mathrm{CH}_{2}\right), 55.7\left(\mathrm{CH}_{3}\right), 57.1(\mathrm{CH}), 80.6(\mathrm{C}), 110.6(2 \mathrm{xCH}), 117.8\left(\mathrm{CH}_{2}\right), 120.5$ $(\mathrm{CH}), 120.8(\mathrm{CH}), 123.7(\mathrm{C}), 125.4(\mathrm{CH}), 125.9(\mathrm{CH}), 126.5(\mathrm{C}), 126.8(\mathrm{CH}), 127.8(\mathrm{CH})$, $128.7(\mathrm{CH}), 128.8(2 \mathrm{xCH}), 132.2(\mathrm{C}), 133.7(\mathrm{C}), 134.7(\mathrm{C}), 135.1(\mathrm{CH}), 154.1(\mathrm{C}), 157.2(\mathrm{C})$. HRMS (EI): $\mathrm{m} / \mathrm{z}$ calcd for $\mathrm{C}_{28} \mathrm{H}_{29} \mathrm{NO}_{3}: 428.2220\left[M+\mathrm{H}^{+}\right]$; found: 428.2219 .

HPLC (Chiralpak ODH, 99.5:0.5 hexane/iPrOH, $\left.1 \mathrm{~mL} \mathrm{~min}^{-1}, 240 \mathrm{~nm}\right) t \mathrm{R}($ major $)=34.69 \mathrm{~min}$, $t \mathrm{R}($ minor $)=46.83 \mathrm{~min}$.

tert-Butyl (S,E)-1-(2-ethoxy-2-oxoethyl)-3-(4-(trifluoromethyl)benzylidene)-1Hbenzo[de]isoquinoline-2(3H)-carboxylate (12bc).

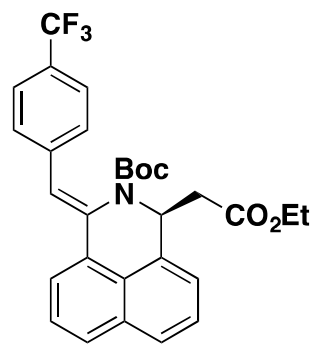

Compound 12bc was synthesized following the general procedure described above starting from $11 \mathbf{b c}(50 \mathrm{mg}, 0.1 \mathrm{mmol})$ affording 12bc as a colorless oil (48 $\mathrm{mg}$,

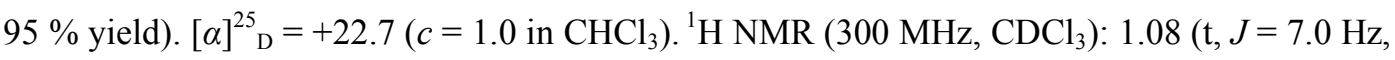
$3 \mathrm{H}), 1.26$ (s, 9H), $2.63(\mathrm{dd}, J=16.0,6.2 \mathrm{~Hz}, 1 \mathrm{H}), 2.92(\mathrm{dd}, J=15.8,8.6 \mathrm{~Hz}, 1 \mathrm{H}), 4.03(\mathrm{~m}, 2 \mathrm{H})$, 6.29 (br s, 1H), 7.29 (br s, 1H), $7.44(\mathrm{~m}, 2 \mathrm{H}), 7.56(\mathrm{~m}, 4 \mathrm{H}), 7.77$ (m, 3H), $7.86(\mathrm{dd}, J=8.3,1.0$ 
$\mathrm{Hz}, 1 \mathrm{H}), 7.92(\mathrm{dd}, J=7.4,1.1 \mathrm{~Hz}, 1 \mathrm{H}) .{ }^{19} \mathrm{~F} \mathrm{NMR}\left(282.4 \mathrm{MHz}, \mathrm{CDCl}_{3}\right) \delta-62.7\left(\mathrm{~s}, \mathrm{CF}_{3}\right) .{ }^{13} \mathrm{C}$ NMR (75.5 MHz, CDCl $)$ : $13.9\left(\mathrm{CH}_{3}\right), 27.9\left(3 \mathrm{xCH}_{3}\right), 29.7(\mathrm{CH}), 41.3\left(\mathrm{CH}_{2}\right), 60.7\left(\mathrm{CH}_{2}\right), 81.3$ (C), $104.4(\mathrm{C}), 121.2(\mathrm{C}), 123.2(\mathrm{C}), 125.1(\mathrm{CH}), 125.2(\mathrm{CH}), 125.9(2 \times \mathrm{CH}), 126.2(\mathrm{C}), 127.3$ $(\mathrm{CH}), 128.8(\mathrm{CH}), 129.1(2 \times \mathrm{CH}), 129.5(\mathrm{CH}), 130.8(\mathrm{C}), 133.6(\mathrm{CH}), 133.9(\mathrm{CH}), 134.8(\mathrm{C})$, 139.2 (C), 153.3 (C), 170.4 (C). HRMS (EI): m/z calcd for $\mathrm{C}_{29} \mathrm{H}_{28} \mathrm{~F}_{3} \mathrm{NO}_{4}: 412.1519\left[M+\mathrm{H}^{+}\right.$Boc]; found: 412.1526 .

HPLC (Chiralpak AD, 92:8 hexane/iPrOH, $\left.1 \mathrm{~mL} \mathrm{~min}^{-1}, 240 \mathrm{~nm}\right) t \mathrm{R}$ (major) $=12.67 \mathrm{~min}$, $t \mathrm{R}($ minor $)=15.63 \mathrm{~min}$.

tert-Butyl (E)-1-allyl-3-(4-(trifluoromethyl)benzylidene)-1H-benzo[de]isoquinoline-2(3H)carboxylate (12cc).

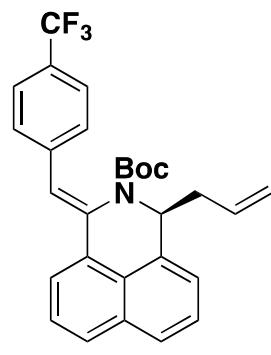

Compound 12cc was synthesized following the general procedure described above starting from 11cc (50 mg, $0.1 \mathrm{mmol}$ ) affording 12ba as a colorless oil (45 mg, $90 \%$ yield). $[\alpha]^{25}=-18.0\left(c=1.0\right.$ in $\left.\mathrm{CHCl}_{3}\right) .{ }^{1} \mathrm{H}$ NMR $\left(300 \mathrm{MHz}, \mathrm{CDCl}_{3}\right): 1.56(\mathrm{~s}, 9 \mathrm{H}), 2.51$ (br s, 1H), 2.76 (br s, 1H), 5.07 (m, 3H), 5.64 (dd, $J=17.2,9.2 \mathrm{~Hz}, 1 \mathrm{H}), 7.14$ (br s, 1H), 7.46 (m, 2H), $7.64(\mathrm{~m}, 3 \mathrm{H}), 7.79(\mathrm{~m}, 3 \mathrm{H}), 7.88(\mathrm{~m}, 2 \mathrm{H}) .{ }^{19} \mathrm{~F} \mathrm{NMR}\left(282.4 \mathrm{MHz}, \mathrm{CDCl}_{3}\right) \delta-62.6\left(\mathrm{~s}, \mathrm{CF}_{3}\right)$. ${ }^{13} \mathrm{C}$ NMR (75.5 MHz, $\left.\mathrm{CDCl}_{3}\right)$ : $28.6\left(\mathrm{CH}_{3}\right), 29.9\left(\mathrm{CH}_{3}\right), 42.5(\mathrm{CH} 2), 50.0(\mathrm{CH}), 79.6(\mathrm{C}), 94.2$ $\left(\mathrm{CH}_{2}\right), 118.7(\mathrm{C}), 124.6(\mathrm{C}), 124.8(2 \times \mathrm{CH}), 125.5(\mathrm{CH}), 125.8(\mathrm{CH}), 127.6(\mathrm{C}), 129.0(\mathrm{C}), 129.8$ (CH), $131.3(\mathrm{CH}), 131.9(\mathrm{CH}), 135.1(\mathrm{C}), 135.8(\mathrm{CH}), 140.3$ (C), 155.3 (C). HRMS (EI): m/z calcd for $\mathrm{C}_{28} \mathrm{H}_{26} \mathrm{~F}_{3} \mathrm{NO}_{2}$ : $466.1998\left[M+\mathrm{H}^{+}\right]$; found: 466.1988 .

HPLC (Chiralpak AD, 99:1 hexane/iPrOH, $\left.1 \mathrm{~mL} \mathrm{~min}^{-1}, 240 \mathrm{~nm}\right) t \mathrm{R}($ major) $=26.29 \mathrm{~min}$, $t \mathrm{R}($ minor $)=31.65 \mathrm{~min}$.

General procedure for the RCEYM reaction. 


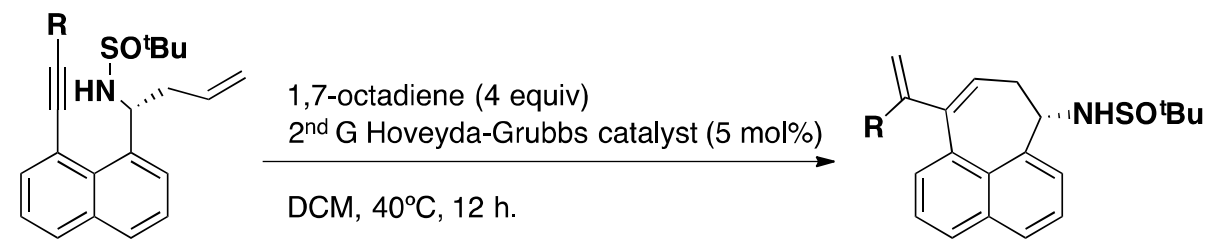

In a round-bottom flask, the corresponding enyne ( $1 \mathrm{mmol}, 1$ equiv) was dissolved in anhydrous dichloromethane $(0.1 \mathrm{M})$ under a nitrogen atmosphere. $2^{\text {nd }}$ generation Hoveyda-Grubbs (H-G II) catalyst ( $5 \mathrm{~mol} \%)$ and 1,7-octadiene (4 equiv) were added (see optimization table S2 below, page S29). The reaction mixture was heated at $45^{\circ} \mathrm{C}$ overnight. The final products were isolated after purification by silica gel flash chromatography with mixtures of hexane:AcOEt (4:1) of increasing polarities as eluent.

\section{2-Methyl-N-(10-(1-phenylvinyl)-7,8-dihydrocyclohepta[de]naphthalen-7-yl)propane-2- sulfinamide (13a).}<smiles>C=C(C)c1ccccc1</smiles>

Compound 13a was synthesized following the general procedure described above starting from $10 \mathrm{ca}(100 \mathrm{mg}, 0.25 \mathrm{mmol})$ affording 13a as a brownish oil (90 $\mathrm{mg}, 90 \%$ yield). $[\alpha]^{25}=+128.9\left(c=1.0\right.$ in $\left.\mathrm{CHCl}_{3}\right) .{ }^{1} \mathrm{H}$ NMR $\left(300 \mathrm{MHz}, \mathrm{CDCl}_{3}\right): 1.12(\mathrm{~s}, 9 \mathrm{H})$, 1.68 (br s, 1H), 2.95 (ddd, $J=14.8,5.0,1.5 \mathrm{~Hz}, 1 \mathrm{H}), 3.06$ (ddd, $J=14.8,8.7,6.4 \mathrm{~Hz}, 1 \mathrm{H}), 5.07$ $(\mathrm{m}, 1 \mathrm{H}), 5.44(\mathrm{~d}, J=1.5 \mathrm{~Hz}, 1 \mathrm{H}), 5.72(\mathrm{~d}, J=1.6 \mathrm{~Hz}, 1 \mathrm{H}), 6.39(\mathrm{~m}, 1 \mathrm{H}), 7.15(\mathrm{~m}, 3 \mathrm{H}), 7.28(\mathrm{~d}$, $J=7.9 \mathrm{~Hz}, 1 \mathrm{H}), 7.34(\mathrm{~m}, 2 \mathrm{H}), 7.44(\mathrm{dd}, J=8.0,7.1 \mathrm{~Hz}, 1 \mathrm{H}), 7.53(\mathrm{dd}, J=7.2,1.7 \mathrm{~Hz}, 1 \mathrm{H})$, $7.65(\mathrm{dd}, J=7.5,1.4 \mathrm{~Hz}, 1 \mathrm{H}), 7.73(\mathrm{dd}, J=8.0,1.4 \mathrm{~Hz}, 1 \mathrm{H}), 7.82(\mathrm{~d}, J=8.0,1.6 \mathrm{~Hz}, 1 \mathrm{H}) .{ }^{13} \mathrm{C}$ NMR (75.5 MHz, $\left.\mathrm{CDCl}_{3}\right)$ : $22.6\left(3 \mathrm{xCH}_{3}\right), 34.1\left(\mathrm{CH}_{2}\right), 55.8(\mathrm{C}), 63.2(\mathrm{CH}), 115.4\left(\mathrm{CH}_{2}\right), 124.9$ $(\mathrm{CH}), \quad 125.0(\mathrm{CH}), 126.6(2 \times \mathrm{CH}), 127.5(\mathrm{CH}), 128.2(3 \times \mathrm{CH}), 128.3(\mathrm{CH}), 129.1(\mathrm{C}), 130.1$ (CH), $130.2(\mathrm{CH}), 131.4(\mathrm{CH}), 133.9(\mathrm{C}), 135.8$ (C), 139.7 (C), $140.1(\mathrm{C}), 145.7$ (C), 152.0 (C). HRMS (EI): $\mathrm{m} / \mathrm{z}$ calcd for $\mathrm{C}_{26} \mathrm{H}_{27} \mathrm{NOS}: 402.1889\left[M+\mathrm{H}^{+}\right]$; found: 402.1886 . 


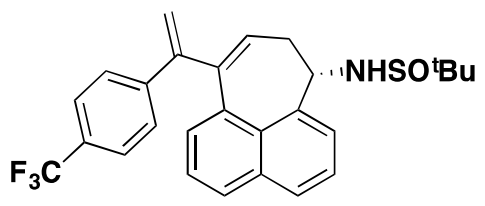

Compound 13b was synthesized following the general procedure described above starting from $10 \mathbf{c c}(100 \mathrm{mg}, 0.21 \mathrm{mmol})$ affording $\mathbf{1 3 b}$ as a brownish oil (78 mg, $78 \%$ yield). $[\alpha]^{25}=-17.3\left(c=1.0\right.$ in $\left.\mathrm{CHCl}_{3}\right) .{ }^{1} \mathrm{H}$ NMR $\left(300 \mathrm{MHz}, \mathrm{CDCl}_{3}\right): 1.12(\mathrm{~s}$, 9H), $3.02(\mathrm{~m}, 2 \mathrm{H}), 4.01(\mathrm{~d}, J=8.7 \mathrm{~Hz}, 1 \mathrm{H}), 5.07(\mathrm{~m}, 1 \mathrm{H}), 5.55(\mathrm{~d}, J=1.4 \mathrm{~Hz}, 1 \mathrm{H}), 5.78(\mathrm{~d}, J=$ $1.3 \mathrm{~Hz}, 1 \mathrm{H}), 6.40(\mathrm{dd}, J=8.3,5.3 \mathrm{~Hz}, 1 \mathrm{H}), 7.30$ (t, $J=8.1 \mathrm{~Hz}, 1 \mathrm{H}), 7.44(\mathrm{~m}, 5 \mathrm{H}), 7.57$ (m, 2H), $7.75(\mathrm{dd}, J=8.2,1.4 \mathrm{~Hz}, 1 \mathrm{H}), 7.83(\mathrm{dd}, J=8.2,1.7 \mathrm{~Hz}, 1 \mathrm{H}) .{ }^{19} \mathrm{~F} \mathrm{NMR}\left(282.4 \mathrm{MHz}, \mathrm{CDCl}_{3}\right) \delta-$ $63.06\left(\mathrm{~s}, \mathrm{CF}_{3}\right) .{ }^{13} \mathrm{C}$ NMR $\left(75.5 \mathrm{MHz}, \mathrm{CDCl}_{3}\right): 21.2\left(3 \mathrm{xCH}_{3}\right), 51.7\left(\mathrm{CH}_{2}\right), 60.5(\mathrm{C}), 75.1(\mathrm{CH})$, $100.2\left(\mathrm{CH}_{2}\right), 113.0(\mathrm{CH}), 122.4(2 \mathrm{xCH}), 122.9(2 \mathrm{xCH}), 129.1(\mathrm{C}), 129.5(3 \times \mathrm{xH}), 129.7$ (2xCH), 130.8 (C), 139.0 (2xC), 139.5 (C), 145.5 (2xC), 152.4 (C). HRMS (EI): m/z calcd for $\mathrm{C}_{27} \mathrm{H}_{26} \mathrm{~F}_{3} \mathrm{NOS}: 470.1760\left[M^{+}+1\right]$; found: 470.1752 .

General procedure for the Pauson-Khand reaction.
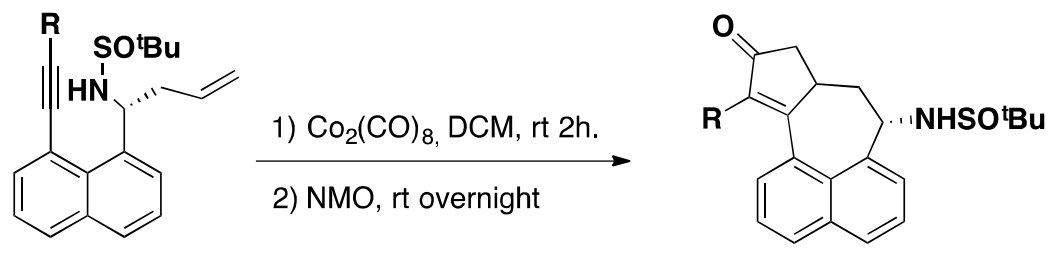

In a round-bottom flask, the corresponding enyne ( $1 \mathrm{mmol}, 1$ equiv) was dissolved in anhydrous toluene $(20 \mathrm{~mL})$ under nitrogen. $\mathrm{Co}_{2}(\mathrm{CO})_{8}$ (1 equiv) was added as a solid, and the mixture was stirred at room temperature until the complete formation of the cobalt-alkyne complex was detected by TLC (typically, 15-30 minutes). NMO was added to the reaction and the mixture was stirred at room temperature overnight. The final products were isolated after purification by silica gel flash chromatography with AcOEt as eluent. 


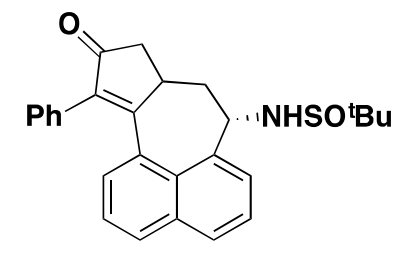

Compound 14a was synthesized following the general procedure described above starting from $10 \mathrm{ca}(100 \mathrm{mg}, 0.25 \mathrm{mmol})$ affording $14 \mathbf{a}$ as a yellow oil (65 $\mathrm{mg}$, $61 \%$ yield). $[\alpha]^{25}=-267.2\left(c=1.0\right.$ in $\left.\mathrm{CHCl}_{3}\right) .{ }^{1} \mathrm{H}$ NMR (300 MHz, $\left.\mathrm{CDCl}_{3}\right): 1.26(\mathrm{~s}, 9 \mathrm{H}), 2.43$ (ddd, $J=12.5,6.6,4.3 \mathrm{~Hz}, 1 \mathrm{H}), 2.60(\mathrm{~m}, 2 \mathrm{H}), 2.86(\mathrm{dd}, J=17.8,6.5 \mathrm{~Hz}, 1 \mathrm{H}), 3.12(\mathrm{dtd}, J=$ 13.1, 6.4, 1,5 Hz, 1H), 3.47 (d, $J=6.3 \mathrm{~Hz}, 1 \mathrm{H}), 5.15(\mathrm{~m}, 1 \mathrm{H}), 7.15$ (d, $J=1.9 \mathrm{~Hz}, 1 \mathrm{H}), 7.22(\mathrm{~m}$, 4H), $7.36(\mathrm{~m}, 2 \mathrm{H}), 7.46$ (t, $J=7.6,1.0 \mathrm{~Hz}, 1 \mathrm{H}), 7.73(\mathrm{~m}, 3 \mathrm{H}) .{ }^{13} \mathrm{C}$ NMR $\left(75.5 \mathrm{MHz}, \mathrm{CDCl}_{3}\right)$ : $22.7\left(3 \mathrm{XCH}_{3}\right), 40.1(\mathrm{CH}), 45.1\left(\mathrm{CH}_{2}\right), 46.7\left(\mathrm{CH}_{2}\right), 56.4(\mathrm{C}), 56.9(\mathrm{CH}), 123.7(\mathrm{CH}), 125.1$ $(\mathrm{CH}), 125.8(\mathrm{CH}), 127.9(\mathrm{CH}), 128.4(2 \times \mathrm{CH}), 128.5(\mathrm{CH}), 129.8(\mathrm{CH}), 130.1(2 \times \mathrm{CH}), 130.3$ (C), 130.5 (CH), 131.4 (C), 133.7 (C), 134.6 (C), 137.7 (C), 139.2 (C), 173.1 (C), 206.0 (C). HRMS (EI): m/z calcd for $\mathrm{C}_{27} \mathrm{H}_{27} \mathrm{NO}_{2} \mathrm{~S}: 430.1835\left[M+1^{+}\right]$; found: 430.1822 .

N-((10S)-3-(2-Methoxyphenyl)-2-oxo-2,10,11,11a-tetrahydro-1H-naphtho[1,8-ef]azulen-10yl)-2-methylpropane-2-sulfinamide (14b)

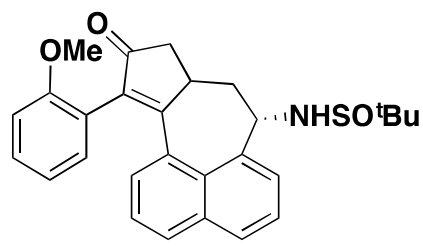

Compound 14b was synthesized following the general procedure described above starting from 10cb $(100 \mathrm{mg}, 0.23 \mathrm{mmol})$ affording $14 \mathbf{b}$ as a yellow oil $(46 \mathrm{mg}$, $43 \%$ yield). $[\alpha]^{25}=-13.1\left(c=1.0\right.$ in $\left.\mathrm{CHCl}_{3}\right) .{ }^{1} \mathrm{H} \mathrm{NMR}\left(300 \mathrm{MHz}, \mathrm{CDCl}_{3}\right): 1.33$ (s, 9H), 2.45 (ddd, $J=16.9,6.7,4.4 \mathrm{~Hz}, 1 \mathrm{H}), 2.59$ (d, $J=17.7 \mathrm{~Hz}, 1 \mathrm{H}), 2.68$ (td, $J=12.2,9.0 \mathrm{~Hz}, 1 \mathrm{H}), 2.90$ (dd, $J=17.9,6.8 \mathrm{~Hz}, 1 \mathrm{H}), 3.19$ (m, 3H), 5.41 (br s, 1H), 6.75 (br s, 1H), 7.05 (br s, 1H), 7.19 (m, 2H), $7.29(\mathrm{~m}, 2 \mathrm{H}), 7.51(\mathrm{t}, J=7.8 \mathrm{~Hz}, 1 \mathrm{H}), 7.76(\mathrm{~m}, 3 \mathrm{H}) .{ }^{13} \mathrm{C} \mathrm{NMR}\left(75.5 \mathrm{MHz}, \mathrm{CDCl}_{3}\right)$ : $22.9\left(3 \mathrm{xCH}_{3}\right), 40.2(\mathrm{CH}), 45.2\left(\mathrm{CH}_{2}\right), 47.3(\mathrm{CH}), 55.2\left(\mathrm{CH}_{3}\right), 56.4\left(\mathrm{CH}_{2}\right), 60.5(\mathrm{C}), 111.4(\mathrm{CH})$, $120.8(\mathrm{C}), 123.6(\mathrm{CH}), 125.1(\mathrm{CH}), 125.7(\mathrm{CH}), 126.8(\mathrm{C}), 128.3(\mathrm{CH}), 129.6(\mathrm{CH}), 129.8(\mathrm{C})$, $130.2(\mathrm{CH}), 131.6(\mathrm{CH}), 134.5(\mathrm{CH}), 135.3(\mathrm{C}), 139.5(\mathrm{CH}), 156.2(\mathrm{C}), 171.2(\mathrm{C}), 175.6(\mathrm{C})$, 206.7 (C). HRMS (EI): $\mathrm{m} / \mathrm{z}$ calcd for $\mathrm{C}_{28} \mathrm{H}_{29} \mathrm{NO}_{3} \mathrm{~S}: 460.1943\left[M+\mathrm{H}^{+}\right]$; found: 460.1941. 
<smiles>CCOCCNC1CC2CC(=O)C(c3ccc(C(F)(F)F)cc3)=C2c2cccc3cccc1c23</smiles>

Compound 14c was synthesized following the general procedure described above starting from 10cc (100 $\mathrm{mg}, 0.21 \mathrm{mmol})$ affording $14 \mathbf{c}$ as a yellow

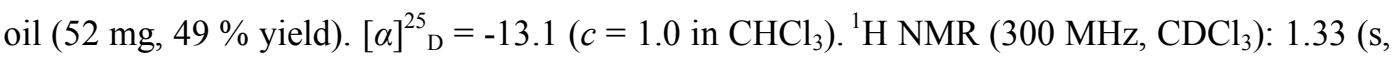
9H), $2.52(\mathrm{ddd}, J=12.7,6.4,4.1 \mathrm{~Hz}, 1 \mathrm{H}), 2.69$ (m, 2H), 2.96 (dd, $J=18.1,6.6 \mathrm{~Hz}, 1 \mathrm{H}), 3.24$ (m, 1H), $3.52(\mathrm{~d}, J=6.1 \mathrm{~Hz}, 1 \mathrm{H}), 5.17(\mathrm{~m}, 1 \mathrm{H}), 7.16(\mathrm{dd}, J=7.3,1.3 \mathrm{~Hz}, 1 \mathrm{H}), 7.25(\mathrm{~m}, 2 \mathrm{H})$, $7.55(\mathrm{~m}, 4 \mathrm{H}), 7.82(\mathrm{~m}, 3 \mathrm{H}) .{ }^{19} \mathrm{~F}$ NMR $\left(282.4 \mathrm{MHz}, \mathrm{CDCl}_{3}\right) \delta-63.1\left(\mathrm{~s}, \mathrm{CF}_{3}\right) .{ }^{13} \mathrm{C}$ NMR $(75.5$ $\left.\mathrm{MHz}, \mathrm{CDCl}_{3}\right): 22.8\left(3 \mathrm{xCH}_{3}\right), 40.3(\mathrm{CH}), 45.1\left(\mathrm{CH}_{2}\right), 46.6\left(\mathrm{CH}_{2}\right), 56.4(\mathrm{CH}), 56.7(\mathrm{C}), 123.9$ $(\mathrm{CH}), 125.2(\mathrm{CH}), 125.4(\mathrm{CH}), 126.0(\mathrm{CH}), 128.7(\mathrm{CH}), 129.1(\mathrm{CH}), 129.6(\mathrm{C}), 129.9(\mathrm{C})$, $130.1(\mathrm{CH}), 130.4(2 \times \mathrm{CH}), 131.0(\mathrm{CH}), 133.0(\mathrm{C}), 134.7$ (C), 135.1 (C), 136.6 (C), 139.1 (C), 174.9 (C), 205.5 (C). HRMS (EI): $\mathrm{m} / \mathrm{z}$ calcd for $\mathrm{C}_{28} \mathrm{H}_{26} \mathrm{~F}_{3} \mathrm{NO}_{2} \mathrm{~S}: 498.1691\left[M+\mathrm{H}^{+}\right]$; found: 498.1709 .

\section{Experimental procedure for the deprotection of $14 \mathrm{~b}$}

To a solution of $\mathbf{1 4 b}$ in anhydrous $\mathrm{MeOH}(0.1 \mathrm{M}), \mathrm{HCl}$ (4M in dioxane, 10 equiv) was added dropwise at $0{ }^{\circ} \mathrm{C}$. The reaction mixture was stirred for 15 minutes, concentrated almost to dryness and diethyl ether was added dropwise. The supernatant was decanted and the thus obtained solid was washed with ether $(2 \times 1 \mathrm{~mL})$ and dried in vacuum.

(7S)-7-amino-11-(2-methoxyphenyl)-7,8,8a,9-tetrahydro-10H-naphtho[1,8-ef]azulen-10-one hydrochloride (XVIII)

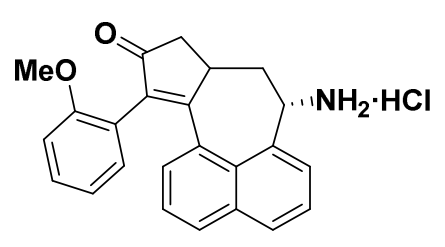

Compound XVIII was synthesized following the procedure described above starting from 14b (46 $\mathrm{mg}, 0.10 \mathrm{mmol})$ affording XVIII as a pale yellow solid (37 mg, $95 \%$ yield). ${ }^{1} \mathrm{H}$ NMR (300 MHz, $\left.\mathrm{CDCl}_{3}\right): 2.37(1 \mathrm{H}, \mathrm{ddd}, J=12.9,5.3,3.5 \mathrm{~Hz}), 2.59$ $(1 \mathrm{H}, \mathrm{d}, J=18.1 \mathrm{~Hz}), 2.81(1 \mathrm{H}, \mathrm{td}, J=12.5,9.2 \mathrm{~Hz}), 3.04(3 \mathrm{H}, \mathrm{dd}, J=18.1,6.6 \mathrm{~Hz}), 3.43-$ $3.37(2 \mathrm{H}, \mathrm{m}), 5.23(1 \mathrm{H}, \mathrm{d}, J=9.9), 6.82(1 \mathrm{H}, \mathrm{s}), 7.03(1 \mathrm{H}, \mathrm{s}), 7.16(1 \mathrm{H}, \mathrm{d}, J=7.0), 7.34-$ 
$7.23(2 \mathrm{H}, \mathrm{m}), 7.49(1 \mathrm{H}, \mathrm{d}, J=7.3 \mathrm{~Hz}), 7.68-7.59(1 \mathrm{H}, \mathrm{m}), 7.88(1 \mathrm{H}, \mathrm{dd}, J=8.2,1.1 \mathrm{~Hz})$, $7.96(1 \mathrm{H}, \mathrm{d}, J=8.2 \mathrm{~Hz}) .{ }^{13} \mathrm{C} \mathrm{NMR}\left(75.5 \mathrm{MHz}, \mathrm{CDCl}_{3}\right)$ : $40.9(\mathrm{CH}), 43.2\left(\mathrm{CH}_{2}\right), 46.3(\mathrm{CH}), 52.9$ $\left(\mathrm{CH}_{2}\right), 55.3\left(\mathrm{OCH}_{3}\right), 112.4(\mathrm{CH}), 121.8(\mathrm{CH}), 122.2(\mathrm{CH}), 126.7(\mathrm{CH}), 126.8(\mathrm{CH}), 130.6(\mathrm{CH})$, $131.0(\mathrm{CH}), 131.6(\mathrm{CH}), 132.7(\mathrm{C}), 135.6(\mathrm{C}), 136.1(\mathrm{CH}), 209.0(\mathrm{CO})$.

Table S1. Screening of gold(I)-catalysts, solvent, temperature and catalyst loading for the intramolecular hydroamination reaction.
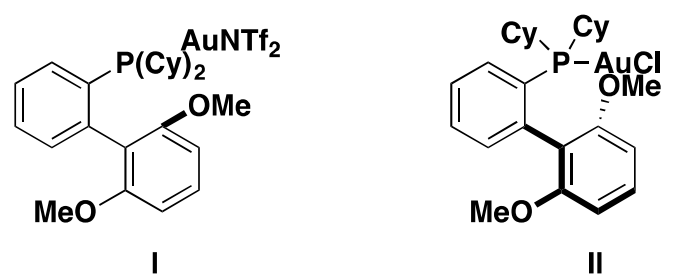

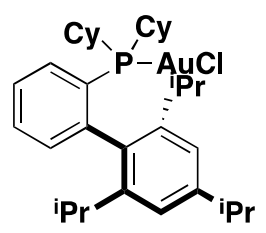

III

\begin{tabular}{lllllll}
\hline Entry & [Au] $\left(\mathbf{m o l}^{-\mathbf{1}}\right)$ & Additive (equiv) & Solvent & T ( $\left.{ }^{\mathbf{0}} \mathbf{C}\right)$ & T (min) & Yield (\%) \\
\hline $\mathbf{1}$ & I (5) & EtOH (5) & MeCN & 30 & 30 & 35 \\
$\mathbf{2}$ & I (5) & EtOH (5) & THF & 30 & 30 & 30 \\
$\mathbf{3}$ & I (5) & EtOH (5) & DCE & 30 & 30 & 47 \\
$\mathbf{4}$ & I (5) & EtOH (5) & DCE & 40 & 15 & 76 \\
$\mathbf{5}$ & II (5) & EtOH (5) & DCE & 40 & 15 & 66 \\
$\mathbf{6}$ & III (5) & EtOH (5) & DCE & 40 & 15 & 66 \\
\hline
\end{tabular}

Table S2. Screening of catalysts, solvent, temperature and catalyst loading for a RCEYM reaction.

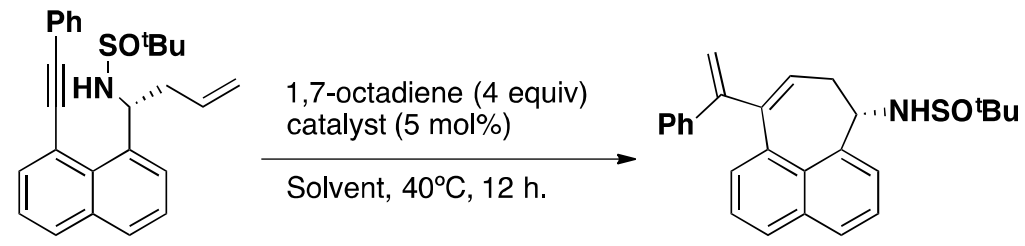

\begin{tabular}{ccccc}
\hline Entry & Catalysts (equiv) & Solvent & T ( $\left.{ }^{\mathbf{C}} \mathbf{c}\right)$ & Yield (\%) \\
\hline $\mathbf{1}$ & Grubbs I & DCM & 40 & - \\
$\mathbf{2}$ & Grubbs II & DCM & 40 & 88 \\
\hline
\end{tabular}




\begin{tabular}{ccccc}
\hline $\mathbf{3}$ & H-G II & DCM & 40 & 90 \\
$\mathbf{4}$ & H-G II & Toluene & 115 & 66 \\
& & & & \\
\hline
\end{tabular}

\section{Computational analysis: Principal Moment of Inertia (PMI)}

\section{General details}

Principal Moment of Inertia (PIM) was performed using Molecular Operating Environment (MOE) software package version 2012.10 from the Chemical Computing Group. Merck molecular force field 94X (MMFF94x), an all-atom force field parameterised for small organic molecules with the Generalised Born solvation model, was used to minimise the energy potential of the library members. A LowModeMD search was employed for the conformation generation. Detailed settings for conformational search are listed below.

$\begin{array}{ll}\text { Rejection Limit } & 100 \\ \text { RMS Gradient } & 0.005 \\ \text { Iteration Limit } & 10000 \\ \text { MM Iteration Limit } & 500 \\ \text { RMSD Limit } & 0.15 \\ \text { Energy window } & 3 \\ \text { Conformation Limit } & 100\end{array}$

Only the conformer with the lowest energy was retained for principal moment of inertia (PMI) calculations. Normalized PMI ratios (I1/I3 and I2/I3) of these conformers were obtained from MOE and then plotted on a triangular graph, with the canonical coordinates $(0,1),(0.5,0.5)$ and $(1,1)$ representing a perfect rod, disc and sphere respectively (Figure 1). 


\section{Compound collections analysed}

A) Collection 1: 19 polycyclic carbo- and heterocyclic compounds from DOS (based on the deprotected virtual derivatives)

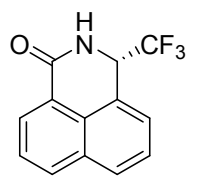

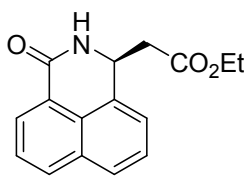

II

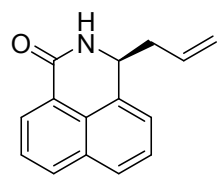

III

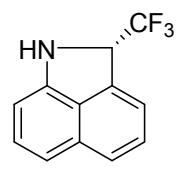

IV

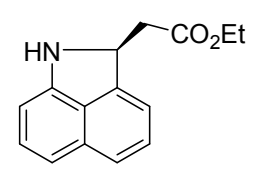

V<smiles>C=C1C[C@H](N)c2cccc3cccc1c23</smiles>

V

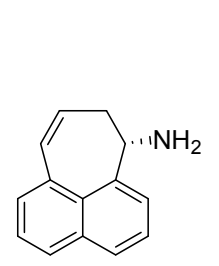

VII<smiles>CCOC(=O)C[C@@H]1N/C(=C\c2ccccc2)c2cccc3cccc1c23</smiles>

VIII

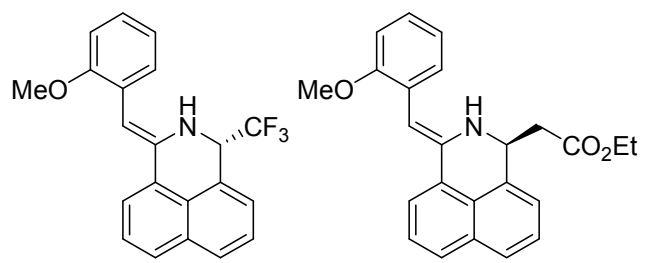

IX
X<smiles>CCOC(=O)CC1N/C(=C\c2ccc(C(F)(F)F)cc2)c2cccc3cccc1c23</smiles>

XI

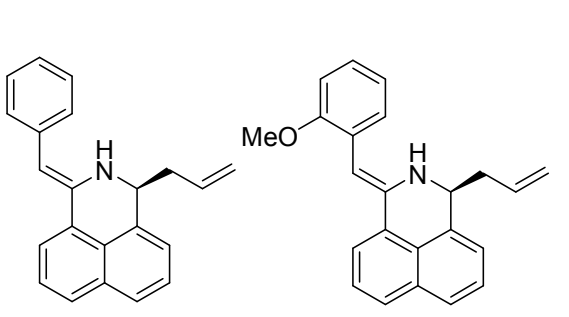

XII

XIII

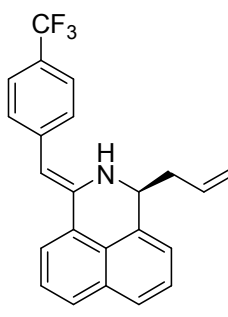

XIV

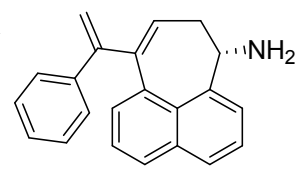

XV<smiles>C=C(C1=CC[C@H](N)c2cccc3cccc1c23)c1ccc(C(F)(F)F)cc1</smiles>

XVI<smiles>N[C@H]1C[C@@H]2CC(=O)C(c3ccccc3)=C2c2cccc3cccc1c23</smiles>

XVII

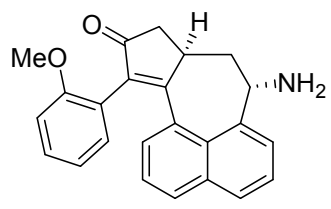

XVIII<smiles>N[C@H]1C[C@H]2CC(=O)C(c3ccc(C(F)(F)F)cc3)=C2c2cccc3cccc1c23</smiles>

XIX

Figure S1

\section{Exemplar scaffold deprotection}

To confirm the validity of the library computational analysis, we demonstrated experimentally that $N$-deprotection reactions were viable. Thus, compounds $\mathbf{4 a}$ and $\mathbf{1 4 b}$ were deprotected achieving I and XVIII uneventfully, see above. 


\section{B) Collection 2: 40 high-profile synthetic drugs currently produced by the pharmaceutical industry}

See: (a) Kopp, F.; Stratton, C. F., Akella, L. B.; Tan, D. S. Nature Chem. Biol. 2012, 8, 358. (b) Bauer, R. A.; Wurst, J. M.; Tan, D. S. Curr. Opin. Chem. Biol. 2010, 14, 308.

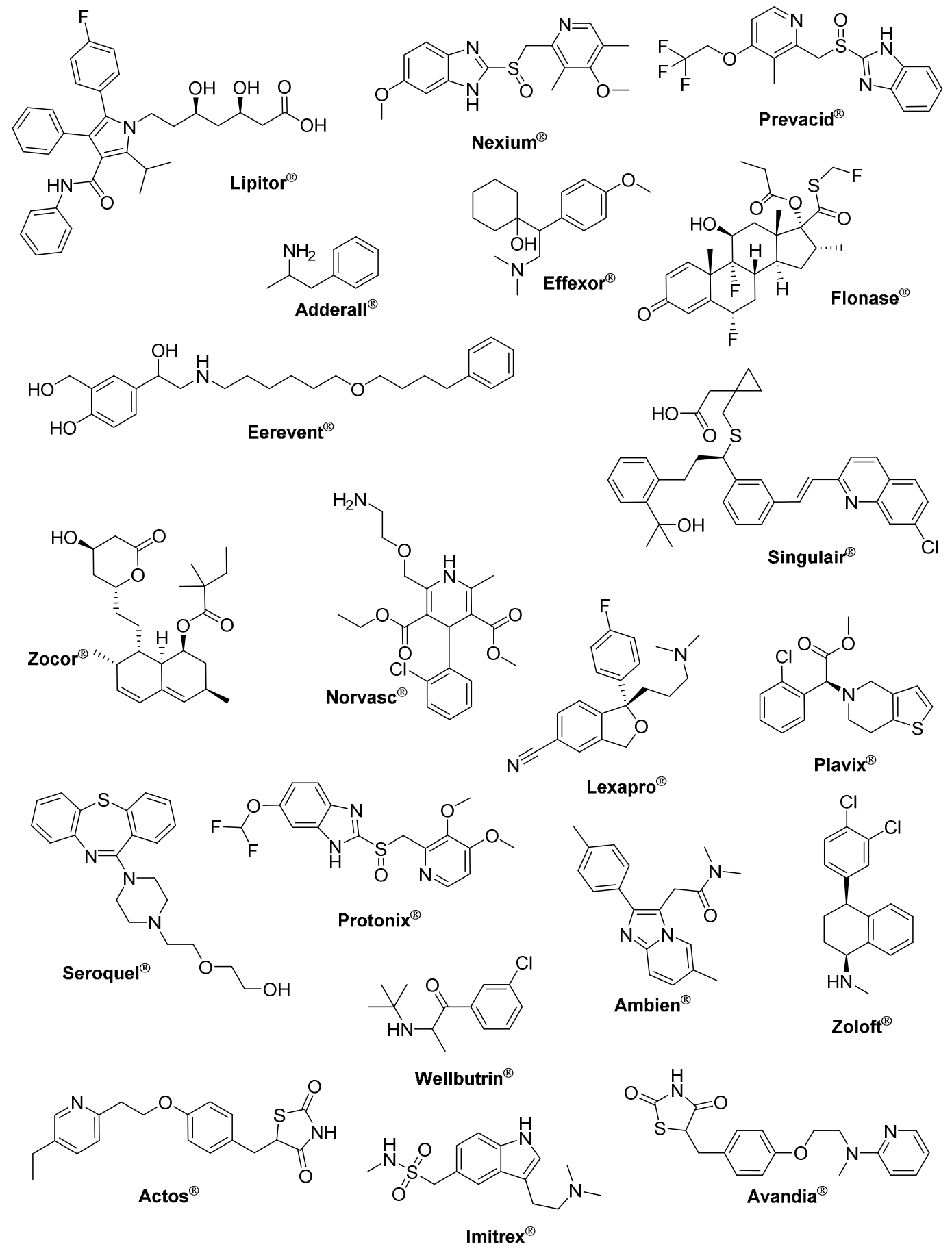

Figure S2. 
<smiles>CCCCCCCCCc1c(C)nc2n(c1=O)CCCC2</smiles>

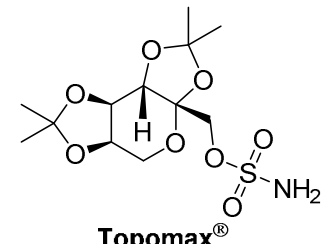<smiles>COCCc1ccc(OCC(O)CNC(C)C)cc1</smiles><smiles>O=C1C(=O)N(c2ccc(F)cc2)[C@H](c2ccc(O)cc2)[C@H]1CC[C@@H](O)c1ccc(F)cc1</smiles><smiles></smiles><smiles>COC(=O)C(c1ccccc1)C1CCCCN1</smiles><smiles>O=C1CCc2cccc(N3CCN(CCCCOc4cccc(Cl)c4Cl)CC3)c2N1</smiles><smiles>CCOC(=O)[C@H](CCc1ccccc1)NC1CCc2ccccc2N(CC(=O)O)C1=O</smiles><smiles>Nc1nnc(-c2cccc(Cl)c2Cl)c(N)n1</smiles><smiles>CC1COc2c(N3CCN(C)CC3)c(F)cc3c(=O)c(C(=O)O)cn1c23</smiles><smiles>Cc1ccc(-c2cc(C(F)(F)F)nn2-c2ccc(S(N)(=O)=O)cc2)cc1</smiles>

Benazepril $^{(}$<smiles>NCCCC(O)(P(=O)(O)O)P(=O)(O)O</smiles><smiles>CC(C)OC(=O)C(C)(C)Oc1ccc(C(=O)c2ccc(Cl)cc2)cc1</smiles>
Tricor $^{\circledR}$<smiles>COc1ccccc1OCCNCC(O)COc1cccc2[nH]c3ccccc3c12</smiles>

Fosamax ${ }^{\circledR}$<smiles>CC(C)C(N)C(=O)OCCOCn1cnc2c(=O)nc(N)[nH]c21</smiles><smiles>CC(C)c1nc(N(C)S(C)(=O)=O)nc(-c2ccc(F)cc2)c1/C=C/C(O)C[C@H](O)CC(=O)O</smiles><smiles>COCCCOc1ccnc(CS(=O)c2nc3ccccc3[nH]2)c1C</smiles>
Aciphex<smiles>CNCCC(Oc1cccc2ccccc12)c1cccs1</smiles><smiles>CCCCC(=O)N(Cc1ccc(-c2ccccc2-c2nn[nH]n2)cc1)C(C(=O)O)C(C)C</smiles><smiles>O=C(O)COCCN1CCN(C(c2ccccc2)c2ccc(Cl)cc2)CC1</smiles>

Figure S2 (cont).

\section{C) Collection 3: 60 randomly selected natural products}

See: (a) Kopp, F.; Stratton, C. F., Akella, L. B.; Tan, D. S. Nature Chem. Biol. 2012, 8, 358. (b) Bauer, R. A.; Wurst, J. M.; Tan, D. S. Curr. Opin. Chem. Biol. 2010, 14, 308. 

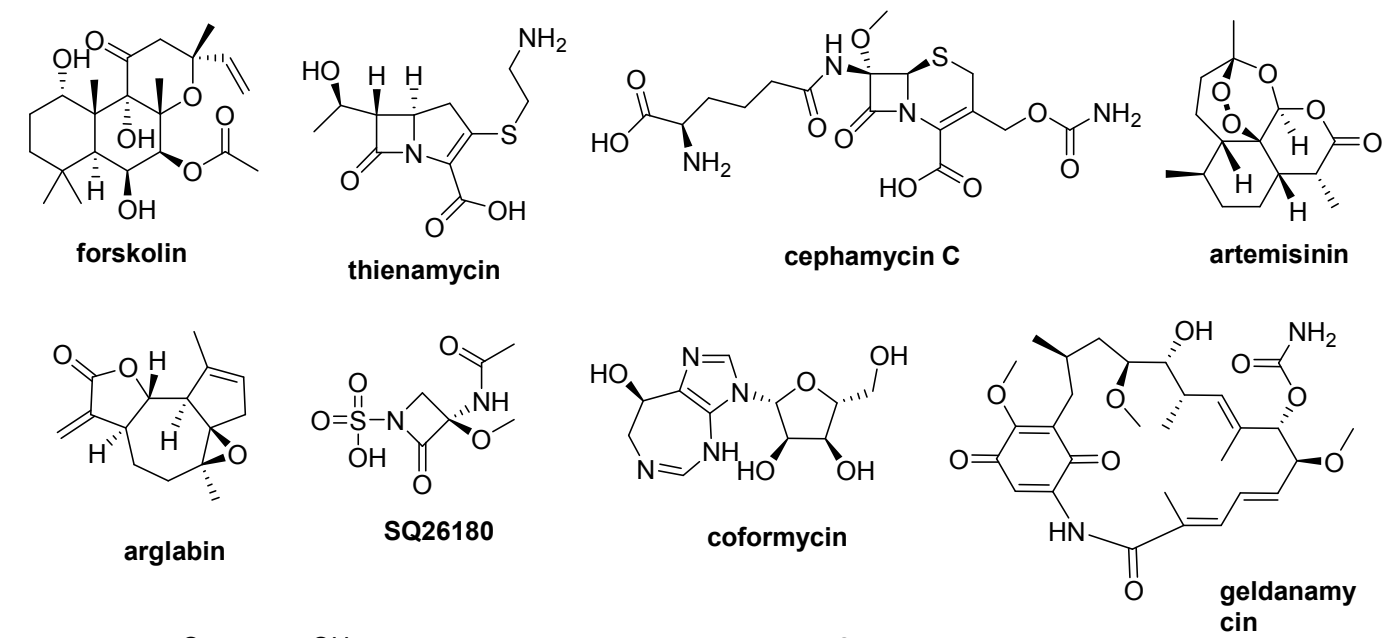<smiles>CCC(C)C(=O)OC1CCC=C2C=CC(C)C(CCC3C[C@@H](O)CC(=O)O3)C21C</smiles>

compactin

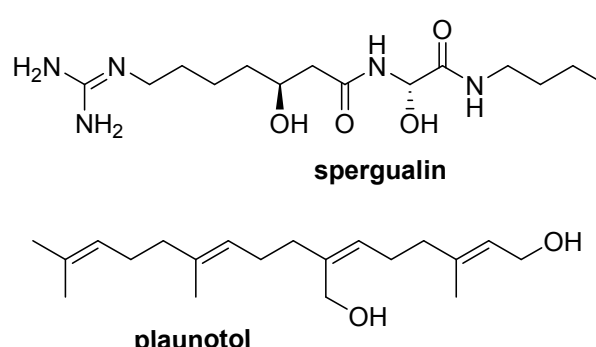

plaunotol<smiles>CNCCCN</smiles><smiles>NC(=O)c1ncn(C2OC(CO)[C@@H](O)[C@H]2O)c1O</smiles>

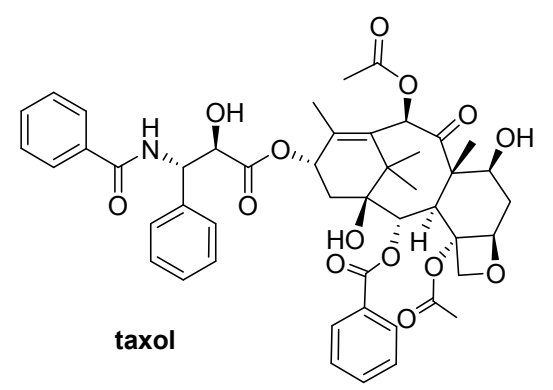

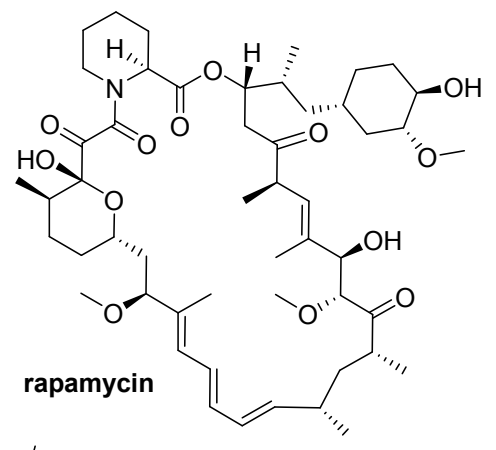<smiles>C=CC1=CN2CCC([C@H](O)c3ccnc4ccc(OC)cc34)[C@@H]2CC1C=C</smiles>

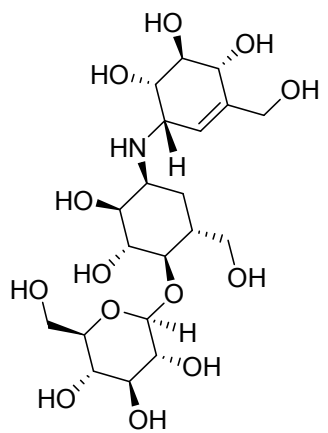

validamycin

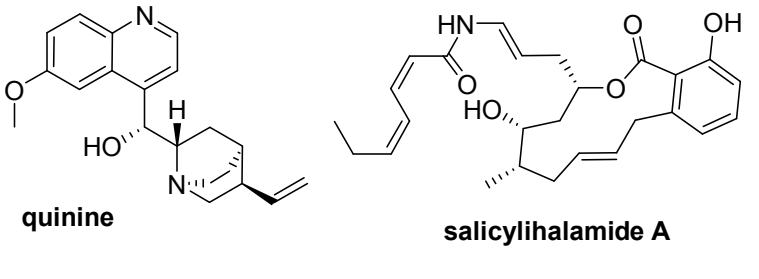

Figure S3. 

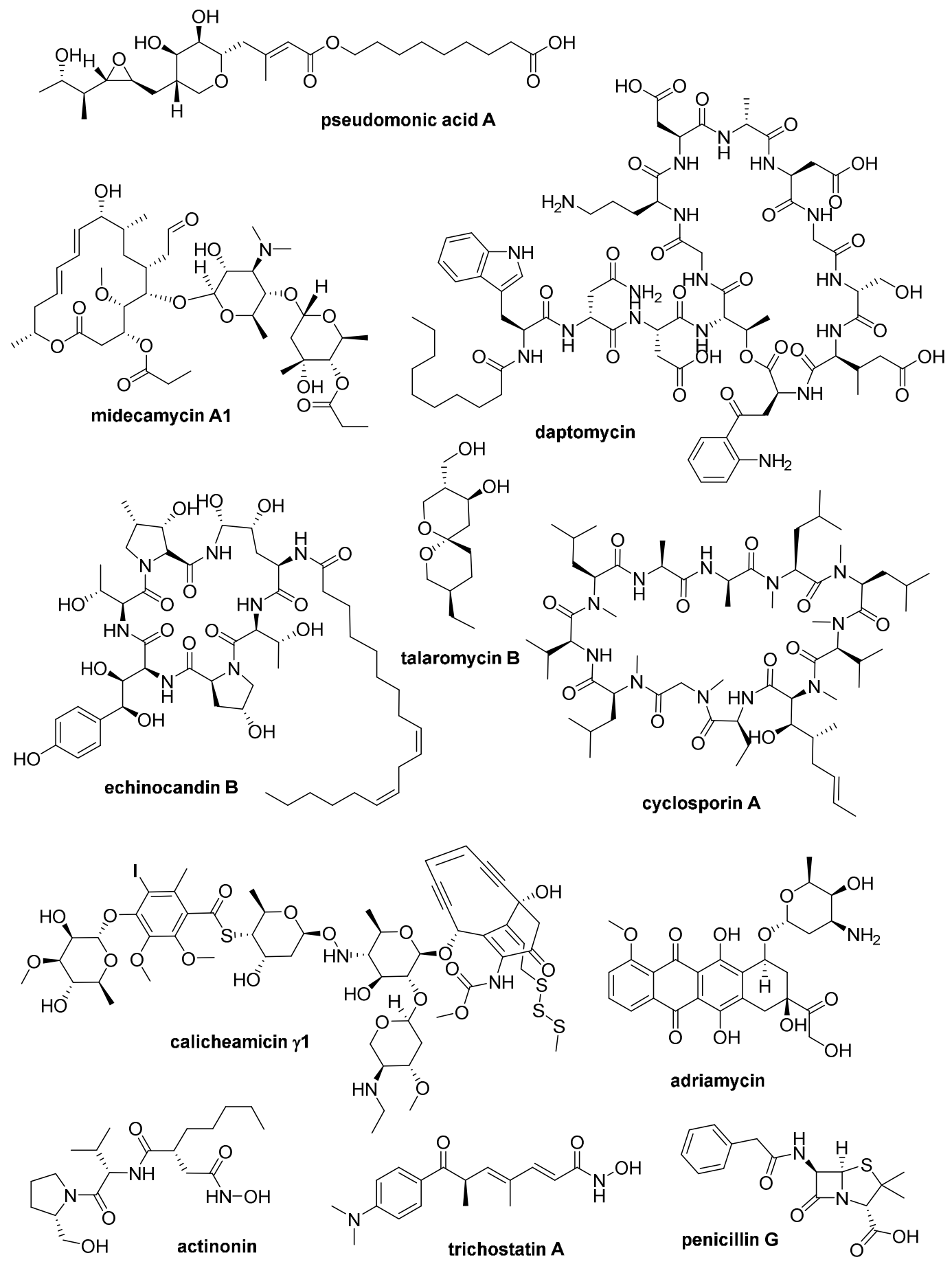

Figure S3 (cont). 


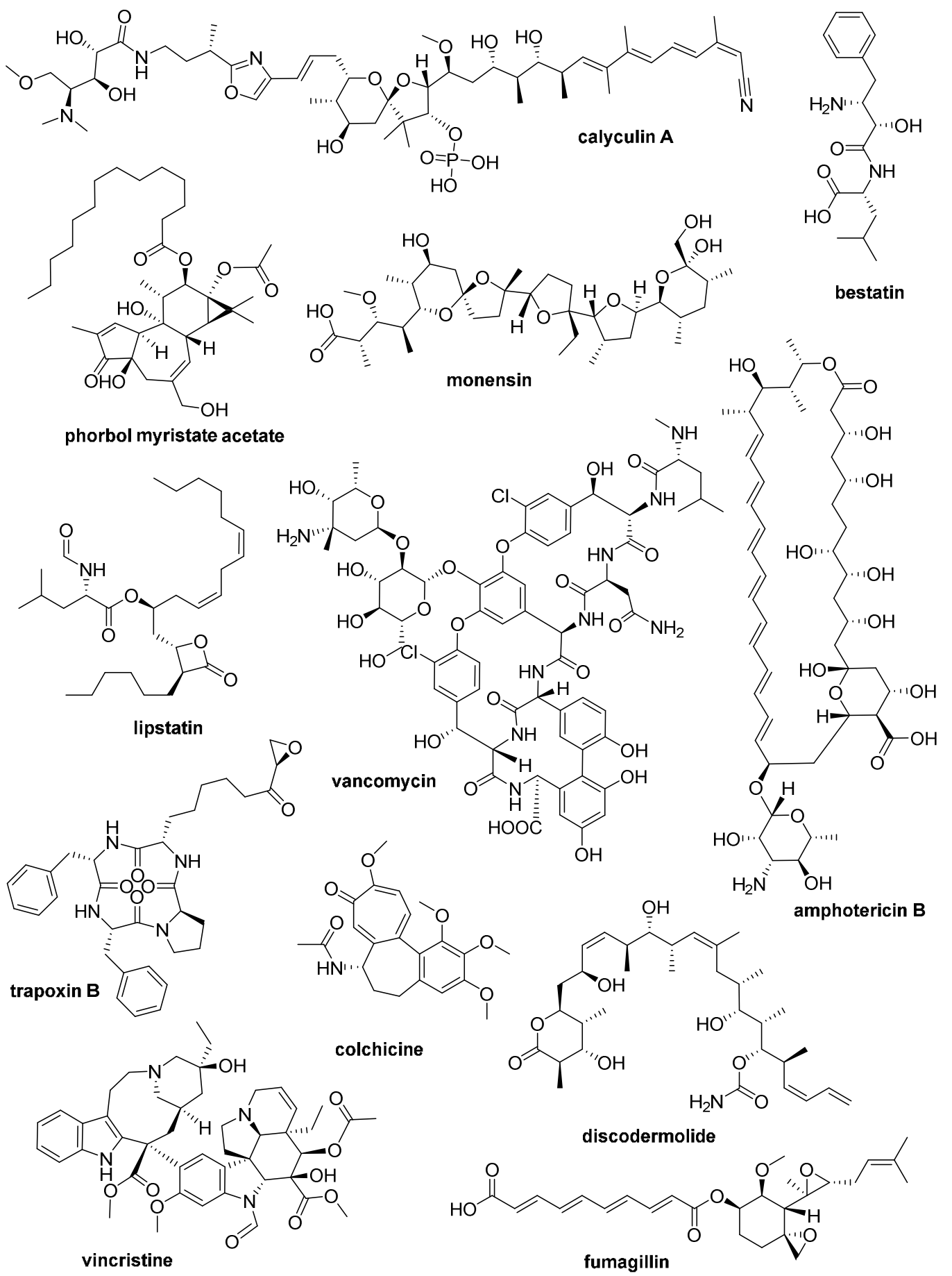

Figure S3 (cont). 


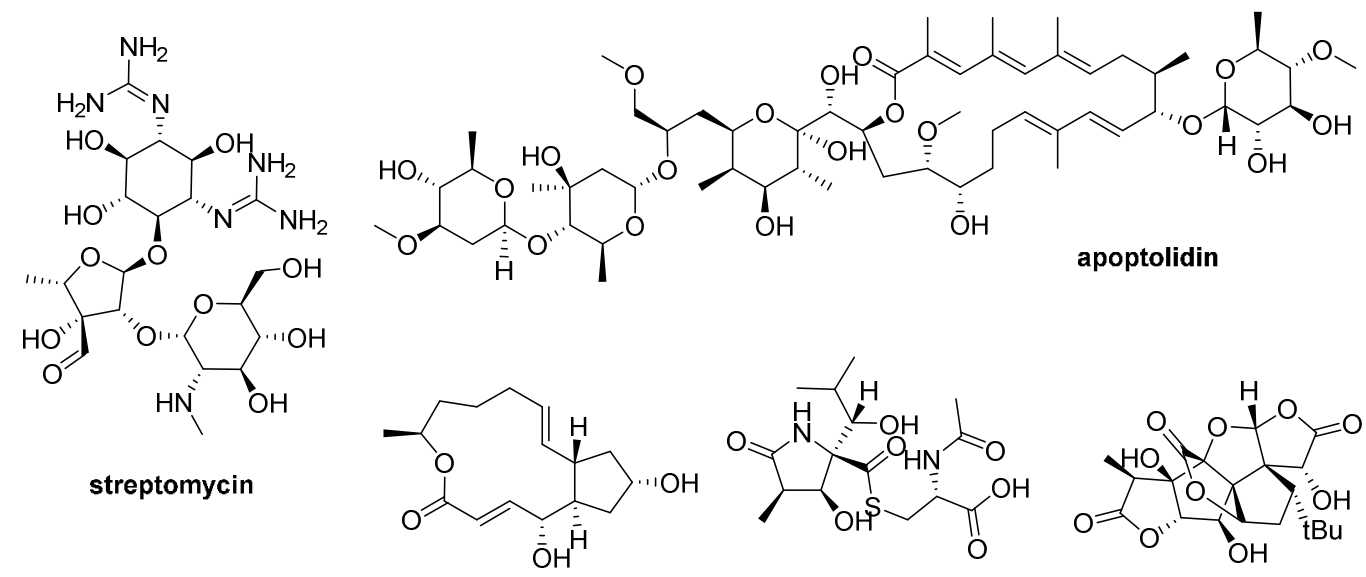<smiles>Cc1c(N)nc([C@@H](CC(N)=O)NC[C@H](N)C(N)=O)nc1C(=O)NCC(=O)N[C@@H](C)[C@H](O)[C@H](C)C(=O)N[C@H](C(=O)NCCc1nc(-c2nc(C(=O)NCCC[S+](C)C)cs2)cs1)[C@@H](C)O</smiles>

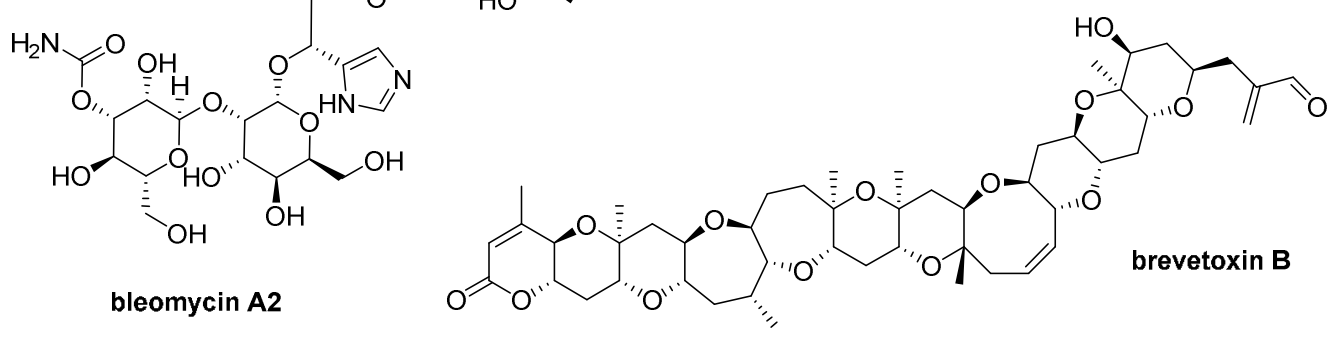

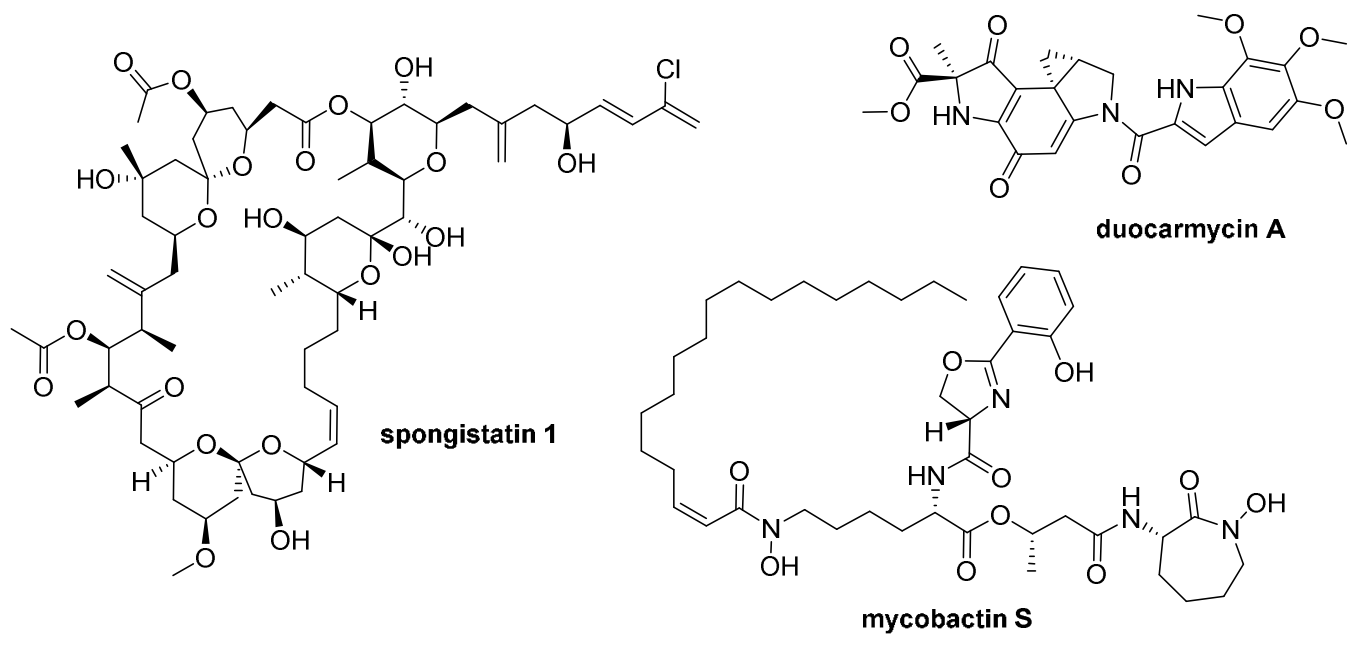

Figure S3 (cont). 

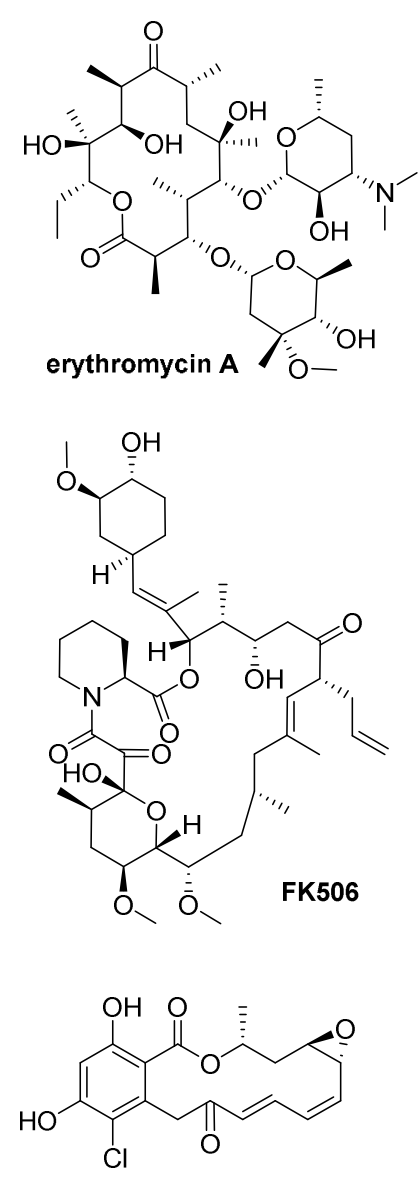

radicicol
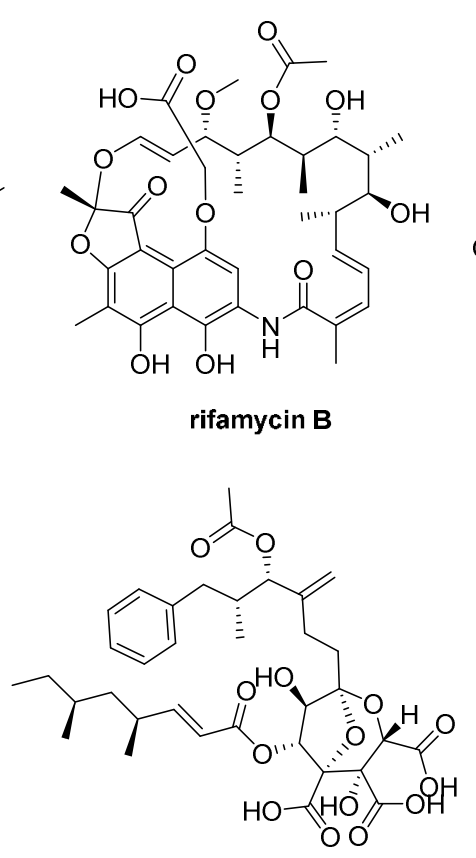

zaragozic acid $\mathbf{A}$

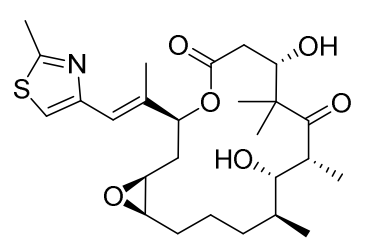

epothilone A
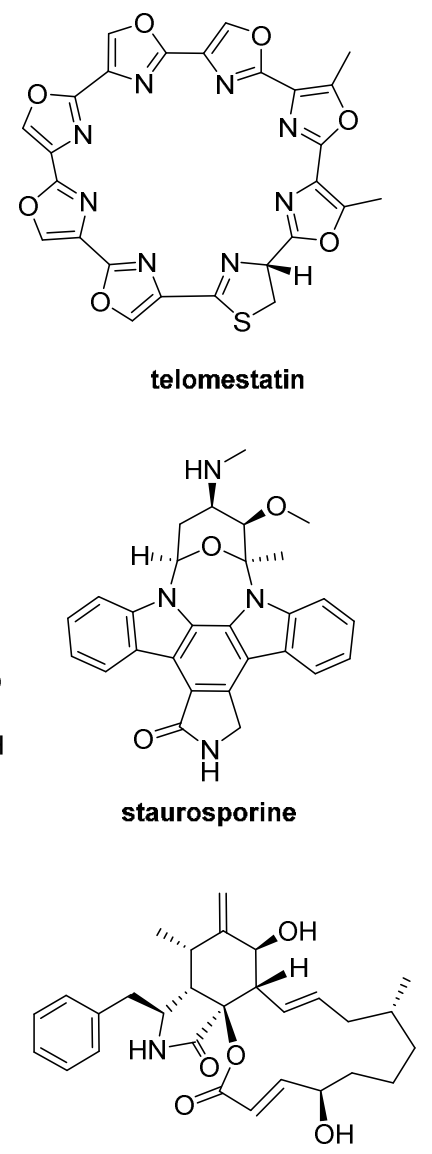

cytochalasin B

Figure S3 (cont).

Table S3 Normalised PMI ratio (npr) values of conformers of the DOS library and two reference collections with the lowest energy $($ energy level $=0 \mathrm{kcal} / \mathrm{mol})$

\section{Collection 1}

\begin{tabular}{cccccc}
\hline Compound & npr1 & npr2 & Compound & npr1 & npr2 \\
\hline I & 0,4442 & 0.6915 & XI & 0.3597 & 0.7764 \\
II & 0.3783 & 0.7913 & XII & 0.3600 & 0.7393 \\
III & 0.5916 & 0.6883 & XIII & 0.3329 & 0.8874 \\
IV & 0.3353 & 0.7525 & XIV & 0.1756 & 0.9117 \\
V & 0.2287 & 0.8779 & $\mathbf{X V}$ & 0.4970 & 0.8918
\end{tabular}




\begin{tabular}{cccccc} 
VI & 0.4836 & 0.5788 & XVI & 0.3693 & 0.9415 \\
VII & 0.4628 & 0.6161 & XVII & 0.4225 & 0.7900 \\
VIII & 0.3281 & 0.7822 & XVIII & 0.5578 & 0.8020 \\
$\mathbf{I X}$ & 0.2424 & 0.8261 & $\mathbf{X I X}$ & 0.3108 & 0.8539 \\
$\mathbf{X}$ & 0.3012 & 0.8347 & & & \\
\hline
\end{tabular}

\section{Collection 2}

\begin{tabular}{|c|c|c|c|c|c|}
\hline Compound & npr1 & npr2 & Compound & npr1 & npr2 \\
\hline Lipitor & 0.3343 & 0.8427 & Topomax & 0.3721 & 0.7907 \\
\hline Nexium & 0.2387 & 0.7858 & Toprol & 0.0854 & 0.9449 \\
\hline Prevacid & 0.1367 & 0.9103 & Zetia & 0.3674 & 0.8320 \\
\hline Flonase & 0.2843 & 0.9666 & Fosamax & 0.6565 & 0.7739 \\
\hline Servent & 0.8749 & 0.9282 & Ability & 0.4836 & 0.6354 \\
\hline Singulair & 0.3979 & 0.7155 & Levaquin & 0.2100 & 0.8459 \\
\hline Effexor & 0.3994 & 0.7418 & Lamictal & 0.2412 & 0.9155 \\
\hline Plavix & 0.3507 & 0.8350 & Celebrex & 0.3738 & 0.6824 \\
\hline Zocor & 0.3846 & 0.7750 & Benazepril & 0.3379 & 0.9290 \\
\hline Norvasc & 0.4396 & 0.8183 & Zyrtec & 0.3208 & 0.8402 \\
\hline Lexapro & 0.4172 & 0.7481 & Coreg & 0.6401 & 0.7545 \\
\hline Seroquel & 0.2078 & 0.9130 & Valtrex & 0.4538 & 0.8509 \\
\hline Protonix & 0.2323 & 0.8070 & Adderall & 0.2184 & 0.9253 \\
\hline Ambien & 0.3818 & 0.6870 & Aciphex & 0.1239 & 0.9138 \\
\hline Actos & 0.1733 & 0.8826 & Cymbalta & 0.3327 & 0.7663 \\
\hline Zoloft & 0.3094 & 0.9498 & Crestor & 0.3525 & 0.8687 \\
\hline
\end{tabular}




\begin{tabular}{ccccccc} 
Wellbutrin & 0.1861 & 0.9472 & Diovan & 0.3509 & 0.9594 \\
Avandia & 0.0876 & 0.9585 & Tricor & 0.1028 & 0.9422 \\
Risperdal & 0.2654 & 0.7797 & Concerta & 0.5477 & 0.6565 \\
Zyprexa & 0.4262 & 0.6254 & Imitrex & 0.2068 & 0.9075 \\
\hline
\end{tabular}

\section{Collection 3}

\begin{tabular}{|c|c|c|c|c|c|}
\hline Compound & npr1 & npr2 & Compound & npr1 & npr2 \\
\hline Taxol & 0.4444 & 0.7558 & Ginkgolide B & 0.4546 & 0.8718 \\
\hline Actinonin & 0.4418 & 0.7805 & Vancomycin & 0.5097 & 0.6634 \\
\hline Discodermolide & 0.1283 & 0.9329 & Amphotericin B & 0.1342 & 0.9067 \\
\hline Validamycin & 0.2010 & 0.9501 & Radicicol & 0.4995 & 0.8727 \\
\hline Monensin & 0.3209 & 0.8721 & Salicylihalamide A & 0.1935 & 0.8944 \\
\hline Calyculin A & 0.4042 & 0.9305 & Telomestatin & 0.4927 & 0.5148 \\
\hline Coformycin & 0.3093 & 0.8134 & Rifamycin B & 0.4922 & 0.7587 \\
\hline Arglabin & 0.3932 & 0.6626 & Apoptolidin & 0.1922 & 0.8755 \\
\hline Mizoribine & 0.2479 & 0.8433 & Midecamycin A1 & 0.3650 & 0.9474 \\
\hline Forskolon & 0.5081 & 0.7477 & Zaragozic acid $A$ & 0.5011 & 0.7235 \\
\hline SQ 26180 & 0.3285 & 0.9244 & Talaromycin B & 0.1546 & 0.9504 \\
\hline Cephamycin C & 0.5613 & 0.6949 & Spongistatin 1 & 0.4968 & 0.8135 \\
\hline Avermectin B1a & 0.3723 & 0.8151 & Brevetoxin B & 0.0410 & 0.9818 \\
\hline Adriamycin & 0.3135 & 0.7704 & Quinine & 0.3647 & 0.8711 \\
\hline $\begin{array}{l}\text { Phorbol } \\
\text { myristate } \\
\text { acetate }\end{array}$ & 0.4660 & 0.7501 & Mycobactin S & 0.3865 & 0.9065 \\
\hline Thienamycin & 0.3015 & 0.8545 & Duocarmycin A & 0.1237 & 0.9519 \\
\hline
\end{tabular}




\begin{tabular}{|c|c|c|c|c|c|}
\hline Cyclosporin A & 0.4809 & 0.8960 & Bleomycin A2 & 0.3651 & 0.9343 \\
\hline FK506 & 0.4472 & 0.8793 & Brefeldin A & 0.3068 & 0.7850 \\
\hline Trapoxin B & 0.7165 & 0.9000 & Cytochalasin B & 0.4974 & 0.6762 \\
\hline Vincristine & 0.5370 & 0.9655 & Epothilone A & 0.3116 & 0.8340 \\
\hline Colchicine & 0.4272 & 0.8346 & Lactacystin & 0.3764 & 0.8347 \\
\hline Trichostatin A & 0.2197 & 0.8615 & Calicheamicin $\gamma 1$ & 0.1774 & 0.9247 \\
\hline Fumagillin & 0.0865 & 0.9668 & Artemisinin & 0.5476 & 0.6380 \\
\hline Staurosporine & 0.4822 & 0.6733 & Compactin & 0.3930 & 0.7646 \\
\hline Erythromycin A & 0.4902 & 0.7797 & Lipstatin & 0.4059 & 0.8457 \\
\hline Streptomycin & 0.3162 & 0.9282 & $\begin{array}{c}\text { Pseudomonic } \\
\text { acid A }\end{array}$ & 0.3896 & 0.6714 \\
\hline Penicillin G & 0.3061 & 0.9575 & Daptomycin & 0.5603 & 0.8611 \\
\hline Sperguallin & 0.2793 & 0.8633 & Bestatin & 0.3910 & 0.7358 \\
\hline Rapamycin & 0.6347 & 0.8330 & Plaunotol & 0.4702 & 0.6467 \\
\hline Echinocandin B & 0.6140 & 0.8022 & Geldanamycin & 0.3478 & 0.7321 \\
\hline
\end{tabular}

Table S4 Chemical structures of conformers of the DOS library and three reference collections in SMILE

Collection 1

\begin{tabular}{|c|l|}
\hline I & $\mathrm{FC}(\mathrm{F})(\mathrm{F})[\mathrm{C} @ \mathrm{H}] 1 \mathrm{NC}(=\mathrm{O}) \mathrm{c} 2 \mathrm{c} 3 \mathrm{c} 1 \mathrm{ccc} 3 \mathrm{ccc} 2$ \\
\hline II & $\mathrm{O}=\mathrm{C} 1 \mathrm{~N}[\mathrm{C} @ \mathrm{H}](\mathrm{c} 2 \mathrm{c} 3 \mathrm{c} 1 \mathrm{ccc} 3 \mathrm{ccc} 2) \mathrm{CC}(\mathrm{OCC})=\mathrm{O}$ \\
\hline III & $\mathrm{O}=\mathrm{C} 1 \mathrm{~N}[\mathrm{C} @ \mathrm{H}](\mathrm{c} 2 \mathrm{c} 3 \mathrm{c} 1 \mathrm{cccc} 3 \mathrm{ccc} 2) \mathrm{CC}=\mathrm{C}$ \\
\hline IV & $\mathrm{FC}(\mathrm{F})(\mathrm{F})[\mathrm{C} @ \mathrm{H}] 1 \mathrm{Nc} 2 \mathrm{c} 3 \mathrm{c} 1 \mathrm{ccc} 3 \mathrm{ccc} 2$ \\
\hline V & $\mathrm{O}(\mathrm{C}(=\mathrm{O}) \mathrm{C}[\mathrm{C} @ @ \mathrm{H}] 1 \mathrm{Nc} 2 \mathrm{c} 3 \mathrm{c} 1 \mathrm{ccc} 3 \mathrm{ccc} 2) \mathrm{CC}$ \\
\hline
\end{tabular}




\begin{tabular}{|c|l|}
\hline VI & $\mathrm{N}[\mathrm{C} @ \mathrm{H}] 1 \mathrm{CC}(\mathrm{c} 2 \mathrm{c} 3 \mathrm{c} 1 \mathrm{ccc} 3 \mathrm{ccc} 2)=\mathrm{C}$ \\
\hline $\mathbf{V I I}$ & $\mathrm{N}[\mathrm{C} @ \mathrm{H}] 1 \mathrm{CC}=\mathrm{Cc} 2 \mathrm{c} 3 \mathrm{c} 1 \mathrm{cccc} 3 \mathrm{ccc} 2$ \\
\hline $\mathbf{V I I I}$ & $\mathrm{FC}(\mathrm{F})(\mathrm{F})[\mathrm{C} @ \mathrm{H}] 1 \mathrm{~N} \backslash \mathrm{C}(\backslash \mathrm{c} 2 \mathrm{c} 3 \mathrm{c} 1 \mathrm{ccc} 3 \mathrm{ccc} 2)=\mathrm{C} / \mathrm{c} 1 \mathrm{ccccc} 1 \mathrm{OC}$ \\
\hline $\mathbf{I X}$ & $\mathrm{O}(\mathrm{C}(=\mathrm{O}) \mathrm{C}[\mathrm{C} @ @ \mathrm{H}] 1 \mathrm{~N} \backslash \mathrm{C}(\backslash \mathrm{c} 2 \mathrm{c} 3 \mathrm{c} 1 \mathrm{cccc} 3 \mathrm{ccc} 2)=\mathrm{C} / \mathrm{c} 1 \mathrm{ccccc} 1) \mathrm{CC}$ \\
\hline $\mathbf{X}$ & $\mathrm{O}(\mathrm{C}) \mathrm{c} 1 \mathrm{cccc} 1 \backslash \mathrm{C}=\mathrm{C} \backslash 1 / \mathrm{N}[\mathrm{C} @ \mathrm{H}](\mathrm{c} 2 \mathrm{c} 3 \mathrm{c} / 1 \mathrm{cccc} 3 \mathrm{ccc} 2) \mathrm{CC}(\mathrm{OCC})=\mathrm{O}$ \\
\hline $\mathbf{X I}$ & $\mathrm{FC}(\mathrm{F})(\mathrm{F}) \mathrm{c} 1 \mathrm{ccc}(\mathrm{cc} 1) \backslash \mathrm{C}=\mathrm{C} \backslash 1 / \mathrm{N}[\mathrm{C} @ \mathrm{H}](\mathrm{c} 2 \mathrm{c} 3 \mathrm{c} / 1 \mathrm{ccc} 3 \mathrm{ccc} 2) \mathrm{CC}(\mathrm{OCC})=\mathrm{O}$ \\
\hline $\mathbf{X I I}$ & $\mathrm{N} \backslash 1[\mathrm{C} @ \mathrm{H}](\mathrm{c} 2 \mathrm{c} 3 \mathrm{c}(\mathrm{cccc} 3 \mathrm{ccc} 2) / \mathrm{C} / 1=\mathrm{C} / \mathrm{c} 1 \mathrm{ccccc} 1) \mathrm{CC}=\mathrm{C}$ \\
\hline $\mathbf{X I I I}$ & $\mathrm{O}(\mathrm{C}) \mathrm{c} 1 \mathrm{cccc} 1 \backslash \mathrm{C}=\mathrm{C} \backslash 1 / \mathrm{N}[\mathrm{C} @ \mathrm{H}](\mathrm{c} 2 \mathrm{c} 3 \mathrm{c} / 1 \mathrm{cccc} 3 \mathrm{ccc} 2) \mathrm{CC}=\mathrm{C}$ \\
\hline $\mathbf{X I V}$ & $\mathrm{FC}(\mathrm{F})(\mathrm{F}) \mathrm{c} 1 \mathrm{ccc}(\mathrm{cc} 1) \backslash \mathrm{C}=\mathrm{C} \backslash 1 / \mathrm{N}[\mathrm{C} @ \mathrm{H}](\mathrm{c} 2 \mathrm{c} 3 \mathrm{c} / 1 \mathrm{ccc} 3 \mathrm{ccc} 2) \mathrm{CC}=\mathrm{C}$ \\
\hline $\mathbf{X V}$ & $\mathrm{N}[\mathrm{C} @ \mathrm{H}] 1 \mathrm{CC}=\mathrm{C}(\mathrm{c} 2 \mathrm{c} 3 \mathrm{c} 1 \mathrm{cccc} 3 \mathrm{ccc} 2) \mathrm{C}(=\mathrm{C}) \mathrm{c} 1 \mathrm{ccccc} 1$ \\
\hline $\mathbf{X V I}$ & $\mathrm{FC}(\mathrm{F})(\mathrm{F}) \mathrm{c} 1 \mathrm{ccc}(\mathrm{cc} 1) \mathrm{C}(=\mathrm{C}) \mathrm{C}=1 \mathrm{c} 2 \mathrm{c} 3 \mathrm{c}(\mathrm{cccc} 3 \mathrm{ccc} 2)[\mathrm{C} @ @ \mathrm{H}](\mathrm{N}) \mathrm{CC}=1$ \\
\hline $\mathbf{X V I I}$ & $\mathrm{O}=\mathrm{C} 1 \mathrm{C}[\mathrm{C} @ @ \mathrm{H}] 2 \mathrm{C}(\mathrm{c} 3 \mathrm{c} 4 \mathrm{c}(\mathrm{cccc} 4 \mathrm{ccc} 3)[\mathrm{C} @ @ \mathrm{H}](\mathrm{N}) \mathrm{C} 2)=\mathrm{C} 1 \mathrm{c} 1 \mathrm{ccccc} 1$ \\
\hline $\mathbf{X V I I I}$ & $\mathrm{O}(\mathrm{C}) \mathrm{c} 1 \mathrm{cccc} 1 \mathrm{C} 1=\mathrm{C} 2[\mathrm{C} @ @ \mathrm{H}](\mathrm{CC} 1=\mathrm{O}) \mathrm{C}[\mathrm{C} @ \mathrm{H}](\mathrm{N}) \mathrm{c} 1 \mathrm{c} 3 \mathrm{c} 2 \mathrm{cccc} 3 \mathrm{ccc} 1$ \\
\hline $\mathbf{X I X}$ & $\mathrm{FC}(\mathrm{F})(\mathrm{F}) \mathrm{c} 1 \mathrm{ccc}(\mathrm{cc} 1) \mathrm{C} 1=\mathrm{C} 2[\mathrm{C} @ @ \mathrm{H}](\mathrm{CC} 1=\mathrm{O}) \mathrm{C}[\mathrm{C} @ \mathrm{H}](\mathrm{N}) \mathrm{c} 1 \mathrm{c} 3 \mathrm{c} 2 \mathrm{cccc} 3 \mathrm{ccc} 1$ \\
\hline
\end{tabular}

\section{Collection 2}

\begin{tabular}{|c|c|}
\hline Lipitor & $\begin{array}{l}\mathrm{Fc1} \operatorname{ccc}(\mathrm{cc} 1)-\mathrm{c} 1 \mathrm{n}(\mathrm{CC}[\mathrm{C} @ @ \mathrm{H}](\mathrm{O}) \mathrm{C}[\mathrm{C} @ @ \mathrm{H}](\mathrm{O}) \mathrm{CC}(=\mathrm{O})[\mathrm{O}- \\
]) \mathrm{c}(\mathrm{C}(\mathrm{C}) \mathrm{C}) \mathrm{c}(\mathrm{C}(=\mathrm{O}) \mathrm{Nc} 2 \mathrm{ccccc} 2) \mathrm{c} 1-\mathrm{c} 1 \mathrm{ccccc} 1\end{array}$ \\
\hline Nexium & $\mathrm{S}(=\mathrm{O})(\mathrm{Cc} 1 \mathrm{ncc}(\mathrm{C}) \mathrm{c}(\mathrm{OC}) \mathrm{c} 1 \mathrm{C}) \mathrm{c} 1[\mathrm{nH}] \mathrm{c} 2 \mathrm{cc}(\mathrm{OC}) \mathrm{ccc} 2 \mathrm{n} 1$ \\
\hline Prevacid & $\mathrm{S}(=\mathrm{O})(\mathrm{Cc} 1 \mathrm{nccc}(\mathrm{OCC}(\mathrm{F})(\mathrm{F}) \mathrm{F}) \mathrm{c} 1 \mathrm{C}) \mathrm{c} 1[\mathrm{nH}] \mathrm{c} 2 \mathrm{c}(\mathrm{n} 1) \mathrm{cccc} 2$ \\
\hline Flonase & $\begin{array}{l}\mathrm{S}(\mathrm{C}=\mathrm{O})[\mathrm{C} @] 1(\mathrm{OC}(=\mathrm{O}) \mathrm{CC})[\mathrm{C} @ @] 2([\mathrm{C} @ \mathrm{H}]([\mathrm{C} @ @ \mathrm{H}] 3 \mathrm{C}[\mathrm{C} @ \mathrm{H}](\mathrm{F}) \mathrm{C} 4=\mathrm{C} \\
\mathrm{C}(=\mathrm{O}) \mathrm{C}=\mathrm{C}[\mathrm{C} @] 4(\mathrm{C})[\mathrm{C} @ @] 3(\mathrm{~F})[\mathrm{C} @ @ \mathrm{H}](\mathrm{O}) \mathrm{C} 2) \mathrm{C}[\mathrm{C} @ \mathrm{H}] 1 \mathrm{C}) \mathrm{C}) \mathrm{CF}\end{array}$ \\
\hline Servent & Oc1ccc(cc1CO)[C@H](O)C[NH2+]CCCCCCOCCCCc1 ccccc1 \\
\hline Singulair & $\mathrm{Clc} 1 \mathrm{cc} 2 \mathrm{nc}(\operatorname{ccc} 2 \mathrm{cc} 1) \backslash \mathrm{C}=\mathrm{Clc} 1 \mathrm{cc}(\mathrm{ccc} 1)[\mathrm{C} @ \mathrm{H}](\mathrm{SCC} 1(\mathrm{CC} 1) \mathrm{CC}(=\mathrm{O})[\mathrm{O}-$ \\
\hline
\end{tabular}




\begin{tabular}{|c|c|}
\hline & ])CCc1 $\operatorname{ccccc} 1 \mathrm{C}(\mathrm{O})(\mathrm{C}) \mathrm{C}$ \\
\hline Effexor & $\mathrm{O}(\mathrm{C}) \mathrm{c} 1 \mathrm{ccc}(\mathrm{cc} 1)[\mathrm{C} @ @ \mathrm{H}](\mathrm{C}[\mathrm{NH}+](\mathrm{C}) \mathrm{C}) \mathrm{C} 1(\mathrm{O}) \mathrm{CCCCC} 1$ \\
\hline Plavix & $\mathrm{Clc1} \operatorname{cccc} 1[\mathrm{C} @ \mathrm{H}]([\mathrm{NH}+] 1 \mathrm{CCc} 2 \mathrm{sccc} 2 \mathrm{C} 1) \mathrm{C}(\mathrm{OC})=\mathrm{O}$ \\
\hline Zocor & $\begin{array}{l}\mathrm{O} 1[\mathrm{C} @ \mathrm{H}](\mathrm{C}[\mathrm{C} @ @ \mathrm{H}](\mathrm{O}) \mathrm{CC} 1=\mathrm{O}) \mathrm{CC}[\mathrm{C} @ @ \mathrm{H}] 1[\mathrm{C} @ @ \mathrm{H}] 2 \mathrm{C}=\mathrm{C}[\mathrm{C} @ @ \mathrm{H}] \\
(\mathrm{C}[\mathrm{C} @ @ \mathrm{H}] 2 \mathrm{OC}(=\mathrm{O}) \mathrm{C}(\mathrm{CC})(\mathrm{C}) \mathrm{C}) \mathrm{C}) \mathrm{C}=\mathrm{C}[\mathrm{C} @ @ \mathrm{H}] 1 \mathrm{C}\end{array}$ \\
\hline Norvasc & $\begin{array}{l}\mathrm{Clc1} \operatorname{ccccc} 1[\mathrm{C} @ @ \mathrm{H}] 1 \mathrm{C}(\mathrm{C}(\mathrm{OCC})=\mathrm{O})=\mathrm{C}(\mathrm{NC}(\mathrm{C})=\mathrm{C} 1 \mathrm{C}(\mathrm{OC})=\mathrm{O}) \mathrm{COCC}[\mathrm{NH} 3+ \\
]\end{array}$ \\
\hline Lexapro & Fc1ccc(cc1)[C@@]1(OCc2cc(ccc12)C\#N)CCC[NH+](C)C \\
\hline Seroquel & $\mathrm{S} 1 \mathrm{c} 2 \mathrm{c}(\mathrm{cccc} 2) \mathrm{C}(=\mathrm{Nc} 2 \mathrm{c} 1 \mathrm{ccc} 2) \mathrm{N} 1 \mathrm{CC}[\mathrm{NH}+](\mathrm{CC} 1) \mathrm{CCOCCO}$ \\
\hline Protonix & $\mathrm{S}(=\mathrm{O})(\mathrm{Cc} 1 \mathrm{nccc}(\mathrm{OC}) \mathrm{c} 1 \mathrm{OC}) \mathrm{c} 1[\mathrm{nH}] \mathrm{c} 2 \mathrm{cc}(\mathrm{OC}(\mathrm{F}) \mathrm{F}) \mathrm{ccc} 2 \mathrm{n} 1$ \\
\hline Ambien & $\mathrm{O}=\mathrm{C}(\mathrm{N}(\mathrm{C}) \mathrm{C}) \mathrm{Cc} 1 \mathrm{n} 2 \mathrm{C}=\mathrm{C}(\mathrm{C}=\mathrm{Cc} 2 \mathrm{nc} 1-\mathrm{c} 1 \mathrm{ccc}(\mathrm{cc} 1) \mathrm{C}) \mathrm{C}$ \\
\hline Actos & $\mathrm{S} 1[\mathrm{C} @ \mathrm{H}](\mathrm{Cc} 2 \mathrm{ccc}(\mathrm{OCC} 3 \mathrm{ncc}(\mathrm{cc} 3) \mathrm{CC}) \mathrm{cc} 2) \mathrm{C}(=\mathrm{O})[\mathrm{N}-] \mathrm{C} 1=\mathrm{O}$ \\
\hline Zoloft & Clc1cc(ccc1Cl)[C@@H]1CC[C@H]([NH2+]C)c2c1 cccc2 \\
\hline Wellbutrin & $\mathrm{Clc1} c \mathrm{c}(\mathrm{ccc} 1) \mathrm{C}(=\mathrm{O})[\mathrm{C} @ \mathrm{H}]([\mathrm{NH} 2+] \mathrm{C}(\mathrm{C})(\mathrm{C}) \mathrm{C}) \mathrm{C}$ \\
\hline Avandia & $\mathrm{S} 1[\mathrm{C} @ @ \mathrm{H}](\mathrm{Cc} 2 \mathrm{ccc}(\mathrm{OCCN}(\mathrm{C}) \mathrm{c} 3 \mathrm{ncccc} 3) \mathrm{cc} 2) \mathrm{C}(=\mathrm{O})[\mathrm{N}-] \mathrm{C} 1=\mathrm{O}$ \\
\hline Risperdal & $\mathrm{Fc} 1 \mathrm{cc} 2 \mathrm{onc}(\mathrm{c} 2 \mathrm{cc} 1) \mathrm{C} 1 \mathrm{CC}[\mathrm{NH}+](\mathrm{CC} 1) \mathrm{CCC}=1 \mathrm{C}(=\mathrm{O}) \mathrm{N} 2 \mathrm{C}(=\mathrm{NC}=1 \mathrm{C}) \mathrm{CCCC} 2$ \\
\hline Zyprexa & $\mathrm{S} 1 \mathrm{C} 2=\mathrm{Nc} 3 \mathrm{c}(\mathrm{NC}(\mathrm{N} 4 \mathrm{CC}[\mathrm{NH}+](\mathrm{CC} 4) \mathrm{C})=\mathrm{C} 2 \mathrm{C}=\mathrm{C} 1 \mathrm{C}) \operatorname{ccc} 3$ \\
\hline Topomax & $\begin{array}{l}\mathrm{S}(\mathrm{OC}[\mathrm{C} @] 12 \mathrm{OC}(\mathrm{O}[\mathrm{C} @ \mathrm{H}] 1[\mathrm{C} @ @ \mathrm{H}] 1 \mathrm{OC}(\mathrm{O}[\mathrm{C} @ @ \mathrm{H}] 1 \mathrm{CO} 2)(\mathrm{C}) \mathrm{C})(\mathrm{C}) \mathrm{C})(=\mathrm{O} \\
)(=\mathrm{O}) \mathrm{N}\end{array}$ \\
\hline Toprol & $\mathrm{O}(\mathrm{C}[\mathrm{C} @ \mathrm{H}](\mathrm{O}) \mathrm{C}[\mathrm{NH} 2+] \mathrm{C}(\mathrm{C}) \mathrm{C}) \mathrm{c} 1 \mathrm{ccc}(\mathrm{cc} 1) \mathrm{CCOC}$ \\
\hline Zetia & $\begin{array}{l}\text { Fc1ccc(cc1)[C@@H](O)CC[C@@H]1[C@H](N(C1=O)c1ccc(F)cc1)c1ccc( } \\
\text { O)cc1 }\end{array}$ \\
\hline Fosamax & $\mathrm{P}(=\mathrm{O})([\mathrm{O}-])([\mathrm{O}-]) \mathrm{C}(\mathrm{P}(=\mathrm{O})([\mathrm{O}-])[\mathrm{O}-])(\mathrm{O}) \mathrm{CCC}[\mathrm{NH} 3+]$ \\
\hline
\end{tabular}




\begin{tabular}{|c|c|}
\hline Ability & $\mathrm{Clc} 1 \mathrm{c}(\mathrm{N} 2 \mathrm{CC}[\mathrm{NH}+](\mathrm{CC} 2) \mathrm{CCCCO} 2 \mathrm{cc} 3 \mathrm{NC}(=\mathrm{O}) \mathrm{CCc} 3 \mathrm{cc} 2) \mathrm{ccc} 1 \mathrm{Cl}$ \\
\hline Levaquin & $\mathrm{Fc} 1 \mathrm{cc} 2 \mathrm{c} 3 \mathrm{~N}(\mathrm{C}=\mathrm{C}(\mathrm{C}(=\mathrm{O})[\mathrm{O}-]) \mathrm{C} 2=\mathrm{O})[\mathrm{C} @ \mathrm{H}](\mathrm{COc} 3 \mathrm{c} 1 \mathrm{~N} 1 \mathrm{CC}[\mathrm{NH}+](\mathrm{CC} 1) \mathrm{C}) \mathrm{C}$ \\
\hline Lamictal & $\operatorname{Clc} 1 \mathrm{c}(\operatorname{cccc} 1 \mathrm{Cl})-\mathrm{c} 1 \mathrm{nnc}(\mathrm{nc} 1 \mathrm{~N}) \mathrm{N}$ \\
\hline Celebrex & $\mathrm{S}(=\mathrm{O})(=\mathrm{O})(\mathrm{N}) \mathrm{c} 1 \mathrm{ccc}(-\mathrm{n} 2 \mathrm{nc}(\mathrm{cc} 2-\mathrm{c} 2 \mathrm{ccc}(\mathrm{cc} 2) \mathrm{C}) \mathrm{C}(\mathrm{F})(\mathrm{F}) \mathrm{F}) \mathrm{cc} 1$ \\
\hline Benazepril & $\begin{array}{l}\mathrm{O}=\mathrm{C} 1 \mathrm{~N}(\mathrm{c} 2 \mathrm{c}(\mathrm{CC}[\mathrm{C} @ @ \mathrm{H}] 1[\mathrm{NH} 2+][\mathrm{C} @ @ \mathrm{H}](\mathrm{CCc} 1 \mathrm{ccccc} 1) \mathrm{C}(\mathrm{OCC})=\mathrm{O}) \mathrm{cccc} 2 \\
) \mathrm{CC}(=\mathrm{O})[\mathrm{O}-]\end{array}$ \\
\hline Zyrtec & $\mathrm{Clc1} \operatorname{ccc}(\mathrm{cc} 1)[\mathrm{C} @ \mathrm{H}]([\mathrm{NH}+] 1 \mathrm{CC}[\mathrm{NH}+](\mathrm{CC} 1) \mathrm{CCOCC}(=\mathrm{O})[\mathrm{O}-]) \operatorname{clcccc} 1$ \\
\hline Coreg & $\mathrm{O}(\mathrm{CC}[\mathrm{NH} 2+] \mathrm{C}[\mathrm{C} @ @ \mathrm{H}](\mathrm{O}) \mathrm{COc} 1 \mathrm{c} 2 \mathrm{c} 3 \mathrm{c}([\mathrm{nH}] \mathrm{c} 2 \mathrm{ccc} 1) \mathrm{cccc} 3) \mathrm{c} 1 \mathrm{ccccc} 1 \mathrm{OC}$ \\
\hline Valtrex & $\mathrm{O}=\mathrm{C} 1 \mathrm{~N}=\mathrm{C}(\mathrm{Nc} 2 \mathrm{n}(\mathrm{cnc} 12) \mathrm{COCCOC}(=\mathrm{O})[\mathrm{C} @ @ \mathrm{H}]([\mathrm{NH} 3+]) \mathrm{C}(\mathrm{C}) \mathrm{C}) \mathrm{N}$ \\
\hline Adderall & [NH3+][C@@H](Cc1 ccccc1)C \\
\hline Aciphex & $\mathrm{S}(=\mathrm{O})(\mathrm{Cc} 1 \mathrm{nccc}(\mathrm{OCCCOC}) \mathrm{c} 1 \mathrm{C}) \mathrm{c} 1[\mathrm{nH}] \mathrm{c} 2 \mathrm{c}(\mathrm{n} 1) \mathrm{cccc} 2$ \\
\hline Cymbalta & s1cccc1[C@@H](Oc1c2c(ccc1)cccc2)CC[NH2+]C \\
\hline Crestor & $\begin{array}{l}\mathrm{S}(=\mathrm{O})(=\mathrm{O})(\mathrm{N}(\mathrm{C}) \mathrm{c} 1 \mathrm{nc}(- \\
\mathrm{c} 2 \mathrm{ccc}(\mathrm{F}) \mathrm{cc} 2) \mathrm{c}(\backslash \mathrm{C}=\mathrm{C} \backslash[\mathrm{C} @ @ \mathrm{H}](\mathrm{O}) \mathrm{C}[\mathrm{C} @ @ \mathrm{H}](\mathrm{O}) \mathrm{CC}(=\mathrm{O})[\mathrm{O}-]) \mathrm{c}(\mathrm{n} 1) \mathrm{C}(\mathrm{C}) \mathrm{C}) \mathrm{C}\end{array}$ \\
\hline Diovan & $\begin{array}{l}\mathrm{O}=\mathrm{C}(\mathrm{N}([\mathrm{C} @ @ \mathrm{H}](\mathrm{C}(\mathrm{C}) \mathrm{C}) \mathrm{C}(=\mathrm{O})[\mathrm{O}-]) \mathrm{Cc} 1 \mathrm{ccc}(\mathrm{cc} 1)-\mathrm{c} 1 \mathrm{ccccc} 1-\mathrm{c} 1 \mathrm{n}[\mathrm{n}- \\
] \mathrm{nn} 1) \mathrm{CCCC}\end{array}$ \\
\hline Tricor & $\mathrm{Clc} 1 \mathrm{ccc}(\mathrm{cc} 1) \mathrm{C}(=\mathrm{O}) \mathrm{c} 1 \mathrm{ccc}(\mathrm{OC}(\mathrm{C}(\mathrm{OC}(\mathrm{C}) \mathrm{C})=\mathrm{O})(\mathrm{C}) \mathrm{C}) \mathrm{cc} 1$ \\
\hline Concerta & $\mathrm{O}(\mathrm{C}(=\mathrm{O})[\mathrm{C} @ \mathrm{H}]([\mathrm{C} @ \mathrm{H}] 1[\mathrm{NH} 2+] \mathrm{CCCC} 1) \mathrm{c} 1 \mathrm{ccccc} 1) \mathrm{C}$ \\
\hline Imitrex & $\mathrm{S}(=\mathrm{O})(=\mathrm{O})(\mathrm{NC}) \mathrm{Cc} 1 \mathrm{cc} 2 \mathrm{c}([\mathrm{nH}] \mathrm{cc} 2 \mathrm{CC}[\mathrm{NH}+](\mathrm{C}) \mathrm{C}) \mathrm{cc} 1$ \\
\hline
\end{tabular}

\section{Collection 3}




\begin{tabular}{|c|c|}
\hline Taxol & $\begin{array}{l}\mathrm{O} 1[\mathrm{C} @ @ \mathrm{H}] 2 \mathrm{C}[\mathrm{C} @ \mathrm{H}](\mathrm{O})[\mathrm{C} @ @] 3([\mathrm{C} @ \mathrm{H}]([\mathrm{C} @ \mathrm{H}](\mathrm{OC}(=\mathrm{O}) \mathrm{c} 4 \mathrm{ccccc} 4)[\mathrm{C} \\
@] 4(\mathrm{O}) \mathrm{C}[\mathrm{C} @ \mathrm{H}](\mathrm{OC}(=\mathrm{O})[\mathrm{C} @ \mathrm{H}](\mathrm{O})[\mathrm{C} @ @ \mathrm{H}](\mathrm{NC}(=\mathrm{O}) \mathrm{c} 5 \mathrm{ccccc} 5) \mathrm{c} 5 \mathrm{ccccc} \\
5) \mathrm{C}(=\mathrm{C}([\mathrm{C} @ @ \mathrm{H}](\mathrm{OC}(=\mathrm{O}) \mathrm{C}) \mathrm{C} 3=\mathrm{O}) \mathrm{C} 4(\mathrm{C}) \mathrm{C}) \mathrm{C})[\mathrm{C} @] 2(\mathrm{OC}(=\mathrm{O}) \mathrm{C}) \mathrm{C} 1) \mathrm{C}\end{array}$ \\
\hline Actinonin & $\begin{array}{l}\mathrm{OC}[\mathrm{C} @ \mathrm{H}] 1 \mathrm{~N}(\mathrm{CCC} 1) \mathrm{C}(=\mathrm{O})[\mathrm{C} @ @ \mathrm{H}](\mathrm{NC}(=\mathrm{O})[\mathrm{C} @ \mathrm{H}](\mathrm{CCCCC}) \mathrm{CC}(=\mathrm{O}) \mathrm{N} \\
[\mathrm{O}-]) \mathrm{C}(\mathrm{C}) \mathrm{C}\end{array}$ \\
\hline Discodermolide & $\begin{array}{l}\mathrm{O} 1[\mathrm{C} @ @ \mathrm{H}](\mathrm{C}[\mathrm{C} @ \mathrm{H}](\mathrm{O}) \backslash \mathrm{C}=\mathrm{C} /[\mathrm{C} @ @ \mathrm{H}]([\mathrm{C} @ \mathrm{H}](\mathrm{O})[\mathrm{C} @ \mathrm{H}](\mathrm{C}=\mathrm{C}(/ \mathrm{C}[\mathrm{C} \\
@ @ \mathrm{H}]([\mathrm{C} @ @ \mathrm{H}](\mathrm{O})[\mathrm{C} @ @ \mathrm{H}]([\mathrm{C} @ @ \mathrm{H}](\mathrm{OC}(=\mathrm{O}) \mathrm{N})[\mathrm{C} @ \mathrm{H}](\mathrm{C}=\mathrm{C} / \mathrm{C}=\mathrm{C}) \\
\mathrm{C}) \mathrm{C}) \mathrm{C}) \backslash \mathrm{C}) \mathrm{C}) \mathrm{C})[\mathrm{C} @ \mathrm{H}](\mathrm{C})[\mathrm{C} @ \mathrm{H}](\mathrm{O})[\mathrm{C} @ @ \mathrm{H}](\mathrm{C}) \mathrm{C}=\mathrm{O}\end{array}$ \\
\hline Validamycin & $\begin{array}{l}\mathrm{O} 1[\mathrm{C} @ \mathrm{H}](\mathrm{CO})[\mathrm{C} @ @ \mathrm{H}](\mathrm{O})[\mathrm{C} @ \mathrm{H}](\mathrm{O})[\mathrm{C} @ @ \mathrm{H}](\mathrm{O})[\mathrm{C} @ @ \mathrm{H}] 1 \mathrm{O}[\mathrm{C} @ \mathrm{H}] 1 \\
{[\mathrm{C} @ \mathrm{H}](\mathrm{O})[\mathrm{C} @ @ \mathrm{H}](\mathrm{O})[\mathrm{C} @ @ \mathrm{H}]([\mathrm{NH} 2+][\mathrm{C} @ \mathrm{H}] 2 \mathrm{C}=\mathrm{C}(\mathrm{CO})[\mathrm{C} @ @ \mathrm{H}](\mathrm{O})} \\
[\mathrm{C} @ \mathrm{H}](\mathrm{O})[\mathrm{C} @ \mathrm{H}] 2 \mathrm{O}) \mathrm{C}[\mathrm{C} @ @ \mathrm{H}] 1 \mathrm{CO}\end{array}$ \\
\hline Monensin & $\begin{array}{l}\mathrm{O} 1[\mathrm{C} @ @ \mathrm{H}]([\mathrm{C} @ \mathrm{H}](\mathrm{C}[\mathrm{C} @ @ \mathrm{H}](\mathrm{C})[\mathrm{C} @] 1(\mathrm{O}) \mathrm{CO}) \mathrm{C})[\mathrm{C} @ @ \mathrm{H}] 1 \mathrm{O}[\mathrm{C} @ @ \\
\mathrm{H}]([\mathrm{C} @] 2(\mathrm{O}[\mathrm{C} @ \mathrm{H}](\mathrm{CC} 2)[\mathrm{C} @] 2(\mathrm{O}[\mathrm{C} @] 3(\mathrm{O}[\mathrm{C} @ \mathrm{H}]([\mathrm{C} @ \mathrm{H}]([\mathrm{C} @ @ \mathrm{H}](\mathrm{O} \\
\mathrm{C})[\mathrm{C} @ @ \mathrm{H}](\mathrm{C}(=\mathrm{O})[\mathrm{O}- \\
]) \mathrm{C}) \mathrm{C})[\mathrm{C} @ \mathrm{H}](\mathrm{C})[\mathrm{C} @ @ \mathrm{H}](\mathrm{O}) \mathrm{C} 3) \mathrm{CC} 2) \mathrm{C}) \mathrm{CC})[\mathrm{C} @ \mathrm{H}](\mathrm{C} 1) \mathrm{C}\end{array}$ \\
\hline Calyculin A & $\begin{array}{l}\mathrm{P}(\mathrm{O}[\mathrm{C} @ \mathrm{H}] 1[\mathrm{C} @ \mathrm{H}](\mathrm{O}[\mathrm{C} @ @] 2(\mathrm{O}[\mathrm{C} @ @ \mathrm{H}](\mathrm{ClC}=\mathrm{C} \backslash \mathrm{c} 3 \mathrm{nc}(\mathrm{oc} 3)[\mathrm{C} @ \mathrm{H}](\mathrm{CC} \\
\mathrm{NC}(=\mathrm{O})[\mathrm{C} @ @ \mathrm{H}](\mathrm{O})[\mathrm{C} @ @ \mathrm{H}](\mathrm{O})[\mathrm{C} @ @ \mathrm{H}]([\mathrm{NH}+](\mathrm{C}) \mathrm{C}) \mathrm{COC}) \mathrm{C})[\mathrm{C} @ @ \\
\mathrm{H}](\mathrm{C})[\mathrm{C} @ \mathrm{H}](\mathrm{O}) \mathrm{C} 2) \mathrm{C} 1(\mathrm{C}) \mathrm{C})[\mathrm{C} @ @ \mathrm{H}](\mathrm{OC}) \mathrm{C}[\mathrm{C} @ \mathrm{H}](\mathrm{O})[\mathrm{C} @ @ \mathrm{H}]([\mathrm{C} @ \mathrm{H}] \\
(\mathrm{O})[\mathrm{C} @ @ \mathrm{H}](\mathrm{C}=\mathrm{C}(\backslash \mathrm{C}(=\mathrm{C} \backslash \mathrm{C}=\mathrm{C} \backslash \mathrm{C}(=\mathrm{C} / \mathrm{CHN}) \backslash \mathrm{C}) \backslash \mathrm{C}) / \mathrm{C}) \mathrm{C}) \mathrm{C})(=\mathrm{O})([\mathrm{O}-])[\mathrm{O}-]\end{array}$ \\
\hline Coformycin & $\begin{array}{l}\mathrm{O} 1[\mathrm{C} @ \mathrm{H}](\mathrm{CO})[\mathrm{C} @ @ \mathrm{H}](\mathrm{O})[\mathrm{C} @ @ \mathrm{H}](\mathrm{O})[\mathrm{C} @ @ \mathrm{H}] 1 \mathrm{n} 1 \mathrm{c} 2 \mathrm{NC}=[\mathrm{NH}+] \mathrm{C}[\mathrm{C} \\
@ @ \mathrm{H}](\mathrm{O}) \mathrm{c} 2 \mathrm{nc} 1\end{array}$ \\
\hline Arglabin & $\begin{array}{l}\mathrm{O} 1[\mathrm{C} @ \mathrm{H}] 2[\mathrm{C} @ @ \mathrm{H}](\mathrm{CC}[\mathrm{C} @ @] 3(\mathrm{O}[\mathrm{C} @] 34[\mathrm{C} @ @ \mathrm{H}] 2 \mathrm{C}(=\mathrm{CC} 4) \mathrm{C}) \mathrm{C}) \mathrm{C}(= \\
\mathrm{C}) \mathrm{C} 1=\mathrm{O}\end{array}$ \\
\hline Mizoribine & O1[C@H](CO)[C@@H](O)[C@@H](O)[C@@H]1n1cnc $(\mathrm{C}(=\mathrm{O}) \mathrm{N}) \mathrm{c} 1 \mathrm{O}$ \\
\hline Forskolon & $\begin{array}{l}\mathrm{O} 1[\mathrm{C} @ @] 2(\mathrm{C})[\mathrm{C} @ @](\mathrm{O})([\mathrm{C} @ @] 3([\mathrm{C} @ @ \mathrm{H}]([\mathrm{C} @ \mathrm{H}](\mathrm{O})[\mathrm{C} @ @ \mathrm{H}] 2 \mathrm{OC}( \\
=\mathrm{O}) \mathrm{C}) \mathrm{C}(\mathrm{CC}[\mathrm{C} @ @ \mathrm{H}] 3 \mathrm{O})(\mathrm{C}) \mathrm{C}) \mathrm{C}) \mathrm{C}(=\mathrm{O}) \mathrm{C}[\mathrm{C} @ @] 1(\mathrm{C}=\mathrm{C}) \mathrm{C}\end{array}$ \\
\hline SQ 26180 & $\mathrm{~S}(=\mathrm{O})(=\mathrm{O})([\mathrm{O}-]) \mathrm{N} 1 \mathrm{C}[\mathrm{C} @](\mathrm{OC})(\mathrm{NC}(=\mathrm{O}) \mathrm{C}) \mathrm{C} 1=\mathrm{O}$ \\
\hline Cephamycin $\mathrm{C}$ & $\begin{array}{l}\mathrm{S} 1[\mathrm{C} @ \mathrm{H}] 2 \mathrm{~N}(\mathrm{C}(\mathrm{C}(=\mathrm{O})[\mathrm{O}- \\
])=\mathrm{C}(\mathrm{C} 1) \mathrm{COC}(=\mathrm{O}) \mathrm{N}) \mathrm{C}(=\mathrm{O})[\mathrm{C} @ @] 2(\mathrm{OC}) \mathrm{NC}(=\mathrm{O}) \mathrm{CCC}[\mathrm{C} @ @ \mathrm{H}]([\mathrm{NH} 3+]\end{array}$ \\
\hline
\end{tabular}




\begin{tabular}{|c|c|}
\hline & $\mathrm{C}(=\mathrm{O})[\mathrm{O}-]$ \\
\hline Avermectin B1a & $\begin{array}{l}\mathrm{O} 1[\mathrm{C} @ @ \mathrm{H}] 2 \mathrm{C}[\mathrm{C} @ @] 3(\mathrm{O}[\mathrm{C} @ \mathrm{H}]([\mathrm{C} @ \mathrm{H}](\mathrm{CC}) \mathrm{C})[\mathrm{C} @ \mathrm{H}](\mathrm{C}=\mathrm{C} 3) \mathrm{C}) \mathrm{O}[\mathrm{C} \\
@ \mathrm{H}](\mathrm{ClC}=\mathrm{C}(/ \mathrm{C}) \backslash \mathrm{C} @ @ \mathrm{H}](\mathrm{O}[\mathrm{C} @ @ \mathrm{H}] 3 \mathrm{O}[\mathrm{C} @ @ \mathrm{H}](\mathrm{C})[\mathrm{C} @ \mathrm{H}](\mathrm{O}[\mathrm{C} @ @ \mathrm{H} \\
] 4 \mathrm{O}[\mathrm{C} @ @ \mathrm{H}](\mathrm{C})[\mathrm{C} @ \mathrm{H}](\mathrm{O})[\mathrm{C} @ @ \mathrm{H}](\mathrm{OC}) \mathrm{C} 4)[\mathrm{C} @ @ \mathrm{H}](\mathrm{OC}) \mathrm{C} 3)[\mathrm{C} @ \mathrm{H}]( \\
\mathrm{C}=\mathrm{ClC}=\mathrm{C} 33 / \mathrm{CO}[\mathrm{C} @ @ \mathrm{H}] 4[\mathrm{C} @ \mathrm{H}](\mathrm{O}) \mathrm{C}(=\mathrm{C}[\mathrm{C} @ \mathrm{H}]([\mathrm{C} @] / 34 \mathrm{O}) \mathrm{C} 1=\mathrm{O}) \mathrm{C}) \mathrm{C} \\
) \mathrm{C} 2\end{array}$ \\
\hline Adriamycin & $\begin{array}{l}\mathrm{O} 1[\mathrm{C} @ @ \mathrm{H}](\mathrm{C})[\mathrm{C} @ @ \mathrm{H}](\mathrm{O})[\mathrm{C} @ @ \mathrm{H}]([\mathrm{NH}+]+\mathrm{C}[\mathrm{C} @ @ \mathrm{H}] 1 \mathrm{O}[\mathrm{C} @ @ \mathrm{H}] 1 \\
\mathrm{c} 2 \mathrm{c}(\mathrm{C}[\mathrm{C} @](\mathrm{O})(\mathrm{C} 1) \mathrm{C}=\mathrm{O}) \mathrm{CO}) \mathrm{c}(\mathrm{O}) \mathrm{c} 1 \mathrm{c}(\mathrm{C}(=\mathrm{O}) \mathrm{c} 3 \mathrm{c}(\mathrm{cccc} 3 \mathrm{OC}) \mathrm{C} 1=\mathrm{O}) \mathrm{c} 2 \mathrm{O}\end{array}$ \\
\hline $\begin{array}{l}\text { Phorbol } \\
\text { myristate } \\
\text { acetate }\end{array}$ & $\begin{array}{l}\mathrm{O}=\mathrm{C} 1[\mathrm{C} @] 2(\mathrm{O})[\mathrm{C} @ @ \mathrm{H}](\mathrm{C}=\mathrm{C} 1 \mathrm{C})[\mathrm{C} @] 1(\mathrm{O})[\mathrm{C} @ \mathrm{H}]([\mathrm{C} @ \mathrm{H}] 3[\mathrm{C} @ @](\mathrm{O} \\
\mathrm{C}(=\mathrm{O}) \mathrm{C})([\mathrm{C} @ \mathrm{H}](\mathrm{OC}(=\mathrm{O}) \mathrm{CCCCCCCCCC})[\mathrm{C} @ \mathrm{H}] 1 \mathrm{C}) \mathrm{C} 3(\mathrm{C}) \mathrm{C}) \mathrm{C}=\mathrm{C}( \\
\mathrm{C} 2) \mathrm{CO}\end{array}$ \\
\hline Thienamycin & $\begin{array}{l}\mathrm{S}(\mathrm{CC}[\mathrm{NH} 3+]) \mathrm{C}=1 \mathrm{C}[\mathrm{C} @ \mathrm{H}] 2 \mathrm{~N}(\mathrm{C}=1 \mathrm{C}(=\mathrm{O})[\mathrm{O}- \\
]) \mathrm{C}(=\mathrm{O})[\mathrm{C} @ @ \mathrm{H}] 2[\mathrm{C} @ \mathrm{H}](\mathrm{O}) \mathrm{C}\end{array}$ \\
\hline Cyclosporin A & $\begin{array}{l}\mathrm{O}=\mathrm{C} 1 \mathrm{~N}(\mathrm{C})[\mathrm{C} @ @ \mathrm{H}]([\mathrm{C} @ \mathrm{H}](\mathrm{O})[\mathrm{C} @ @ \mathrm{H}](\mathrm{ClC}=\mathrm{ClC}) \mathrm{C}) \mathrm{C}=\mathrm{O}) \mathrm{N}[\mathrm{C} @ @ \mathrm{H}] \\
(\mathrm{CC}) \mathrm{C}(=\mathrm{O}) \mathrm{N}(\mathrm{CC}(=\mathrm{O}) \mathrm{N}(\mathrm{C})[\mathrm{C} @ @ \mathrm{H}](\mathrm{CC}(\mathrm{C}) \mathrm{C}) \mathrm{C}(=\mathrm{O}) \mathrm{N}[\mathrm{C} @ @ \mathrm{H}](\mathrm{C}(\mathrm{C}) \mathrm{C}) \\
\mathrm{C}(=\mathrm{O}) \mathrm{N}(\mathrm{C})[\mathrm{C} @ @ \mathrm{H}](\mathrm{CC}(\mathrm{C}) \mathrm{C}) \mathrm{C}(=\mathrm{O}) \mathrm{N}[\mathrm{C} @ @ \mathrm{H}](\mathrm{C}) \mathrm{C}(=\mathrm{O}) \mathrm{N}[\mathrm{C} @ \mathrm{H}](\mathrm{C}) \mathrm{C}( \\
=\mathrm{O}) \mathrm{N}(\mathrm{C})[\mathrm{C} @ @ \mathrm{H}](\mathrm{CC}(\mathrm{C}) \mathrm{C}) \mathrm{C}(=\mathrm{O}) \mathrm{N}(\mathrm{C})[\mathrm{C} @ @ \mathrm{H}](\mathrm{CC}(\mathrm{C}) \mathrm{C}) \mathrm{C}(=\mathrm{O}) \mathrm{N}(\mathrm{C})[\mathrm{C} \\
@ \mathrm{H}] 1 \mathrm{C}(\mathrm{C}) \mathrm{C}) \mathrm{C}\end{array}$ \\
\hline FK506 & $\begin{array}{l}\mathrm{O} 1[\mathrm{C} @ \mathrm{H}](/ \mathrm{C}(=\mathrm{C} /[\mathrm{C} @ \mathrm{H}] 2 \mathrm{C}[\mathrm{C} @ @ \mathrm{H}](\mathrm{OC})[\mathrm{C} @ \mathrm{H}](\mathrm{O}) \mathrm{CC} 2) / \mathrm{C})[\mathrm{C} @ \mathrm{H}](\mathrm{C})[ \\
\mathrm{C} @ @ \mathrm{H}](\mathrm{O}) \mathrm{CC}(=\mathrm{O})[\mathrm{C} @ @ \mathrm{H}](\mathrm{C}=\mathrm{C}(\mathrm{C}[\mathrm{C} @ @ \mathrm{H}](\mathrm{C}[\mathrm{C} @ \mathrm{H}](\mathrm{OC})[\mathrm{C} @ \mathrm{H}] 2 \\
\mathrm{O}[\mathrm{C} @](\mathrm{O})([\mathrm{C} @ @ \mathrm{H}](\mathrm{C}[\mathrm{C} @ @ \mathrm{H}] 2 \mathrm{OC}) \mathrm{C}) \mathrm{C}(=\mathrm{O}) \mathrm{C}(=\mathrm{O}) \mathrm{N} 2[\mathrm{C} @ \mathrm{H}](\mathrm{CCC} \\
\mathrm{C} 2) \mathrm{C} 1=\mathrm{O}) \mathrm{C}) / \mathrm{C}) \mathrm{CC}=\mathrm{C}\end{array}$ \\
\hline Trapoxin B & $\begin{array}{l}\mathrm{O} 1 \mathrm{C}[\mathrm{C} @ \mathrm{H}] 1 \mathrm{C}(=\mathrm{O}) \mathrm{CCCCC}[\mathrm{C} @ @ \mathrm{H}] 1 \mathrm{NC}(=\mathrm{O})[\mathrm{C} @ @ \mathrm{H}] 2 \mathrm{~N}(\mathrm{CCC} 2) \mathrm{C}(=\mathrm{O} \\
)[\mathrm{C} @ \mathrm{H}](\mathrm{NC}(=\mathrm{O})[\mathrm{C} @ @ \mathrm{H}](\mathrm{NC} 1=\mathrm{O}) \mathrm{Cc} 1 \mathrm{ccccc} 1) \mathrm{Cc} 1 \mathrm{ccccc} 1\end{array}$ \\
\hline Vincristine & $\begin{array}{l}\mathrm{O}(\mathrm{C}) \mathrm{c} 1 \mathrm{cc} 2 \mathrm{~N}([\mathrm{C} @ @ \mathrm{H}] 3[\mathrm{C} @] 4([\mathrm{C} @ \mathrm{H}] 5[\mathrm{NH}+](\mathrm{CC}=\mathrm{C}[\mathrm{C} @ @] 5(\mathrm{CC})[\mathrm{C} @ \\
@ \mathrm{H}](\mathrm{OC}(=\mathrm{O}) \mathrm{C})[\mathrm{C} @] 3(\mathrm{O}) \mathrm{C}(\mathrm{OC})=\mathrm{O}) \mathrm{CC} 4) \mathrm{c} 2 \mathrm{cc} 1[\mathrm{C} @ @] 1(\mathrm{c} 2[\mathrm{nH}] \mathrm{c} 3 \mathrm{c}(\mathrm{c} 2 \mathrm{C} \\
\mathrm{C}[\mathrm{N} @ \mathrm{H}+] 2 \mathrm{C}[\mathrm{C} @ @](\mathrm{O})(\mathrm{C}[\mathrm{C} @ @ \mathrm{H}](\mathrm{C} 1) \mathrm{C} 2) \mathrm{CC}) \mathrm{ccc} 3) \mathrm{C}(\mathrm{OC})=\mathrm{O}) \mathrm{C}=\mathrm{O}\end{array}$ \\
\hline Colchicine & $\begin{array}{l}\mathrm{O}(\mathrm{C}) \mathrm{C} 1=\mathrm{CC}=\mathrm{C} 2 \mathrm{c} 3 \mathrm{c}(\mathrm{cc}(\mathrm{OC}) \mathrm{c}(\mathrm{OC}) \mathrm{c} 3 \mathrm{OC}) \mathrm{CC}[\mathrm{C} @ \mathrm{H}](\mathrm{NC}(=\mathrm{O}) \mathrm{C}) \mathrm{C} 2=\mathrm{CC} 1= \\
\mathrm{O}\end{array}$ \\
\hline
\end{tabular}




\begin{tabular}{|c|c|}
\hline Trichostatin A & $\mathrm{O}=\mathrm{C}([\mathrm{C} @ @ \mathrm{H}](\backslash \mathrm{C}=\mathrm{C}(\backslash \mathrm{C}=\mathrm{C} \backslash \mathrm{C}(=\mathrm{O}) \mathrm{N}[\mathrm{O}-]) / \mathrm{C}) \mathrm{C}) \mathrm{c} 1 \mathrm{ccc}(\mathrm{N}(\mathrm{C}) \mathrm{C}) \mathrm{cc} 1$ \\
\hline Fumagillin & $\begin{array}{l}\mathrm{O} 1[\mathrm{C} @](\mathrm{C})([\mathrm{C} @ \mathrm{H}] 2[\mathrm{C} @ \mathrm{H}](\mathrm{OC})[\mathrm{C} @ \mathrm{H}](\mathrm{OC}(=\mathrm{O}) \backslash \mathrm{C}=\mathrm{C} \backslash \mathrm{C}=\mathrm{C} \backslash \mathrm{C}=\mathrm{C} \backslash \mathrm{C}=\mathrm{C} \backslash \\
\mathrm{C}(=\mathrm{O})[\mathrm{O}-]) \mathrm{CC}[\mathrm{C} @ @] 23 \mathrm{OC} 3)[\mathrm{C} @ \mathrm{H}] 1 \mathrm{C} \backslash \mathrm{C}=\mathrm{C}(\backslash \mathrm{C}) / \mathrm{C}\end{array}$ \\
\hline Staurosporine & $\begin{array}{l}\mathrm{O} 1[\mathrm{C} @ \mathrm{H}] 2 \mathrm{n} 3 \mathrm{c} 4 \mathrm{c}(\mathrm{c} 5 \mathrm{c}(\mathrm{CNC} 5=\mathrm{O}) \mathrm{c} 5 \mathrm{c} 6 \mathrm{c}(\mathrm{n}(\mathrm{c} 45)[\mathrm{C} @] 1(\mathrm{C})[\mathrm{C} @ \mathrm{H}](\mathrm{OC})[\mathrm{C} @ \\
\mathrm{H}]([\mathrm{NH} 2+] \mathrm{C}) \mathrm{C} 2) \mathrm{cccc} 6) \mathrm{c} 1 \mathrm{c} 3 \mathrm{cccc} 1\end{array}$ \\
\hline Erythromycin A & $\begin{array}{l}\mathrm{O} 1[\mathrm{C} @ \mathrm{H}](\mathrm{CC})[\mathrm{C} @](\mathrm{O})(\mathrm{C})[\mathrm{C} @ \mathrm{H}](\mathrm{O})[\mathrm{C} @ @ \mathrm{H}](\mathrm{C}) \mathrm{C}(=\mathrm{O})[\mathrm{C} @ @ \mathrm{H}](\mathrm{C}[\mathrm{C} \\
@](\mathrm{O})(\mathrm{C})[\mathrm{C} @ \mathrm{H}](\mathrm{O}[\mathrm{C} @ @ \mathrm{H}] 2 \mathrm{O}[\mathrm{C} @ @ \mathrm{H}](\mathrm{C}[\mathrm{C} @ \mathrm{H}]([\mathrm{NH}+(\mathrm{C}) \mathrm{C})[\mathrm{C} @ \mathrm{H}] 2 \\
\mathrm{O}) \mathrm{C})[\mathrm{C} @ @ \mathrm{H}](\mathrm{C})[\mathrm{C} @ \mathrm{H}](\mathrm{O}[\mathrm{C} @ @ \mathrm{H}] 2 \mathrm{O}[\mathrm{C} @ @ \mathrm{H}](\mathrm{C})[\mathrm{C} @ \mathrm{H}](\mathrm{O})[\mathrm{C} @](\mathrm{O} \\
\mathrm{C})(\mathrm{C} 2) \mathrm{C})[\mathrm{C} @ @ \mathrm{H}](\mathrm{C}) \mathrm{C} 1=\mathrm{O}) \mathrm{C}\end{array}$ \\
\hline Streptomycin & $\begin{array}{l}\mathrm{O} 1[\mathrm{C} @ @ \mathrm{H}](\mathrm{CO})[\mathrm{C} @ \mathrm{H}](\mathrm{O})[\mathrm{C} @ @ \mathrm{H}](\mathrm{O})[\mathrm{C} @ \mathrm{H}]([\mathrm{NH} 2+] \mathrm{C})[\mathrm{C} @ @ \mathrm{H}] 1 \mathrm{O}[ \\
\mathrm{C} @ @ \mathrm{H}] 1[\mathrm{C} @ @](\mathrm{O})(\mathrm{C}=\mathrm{O})[\mathrm{C} @ @ \mathrm{H}](\mathrm{O}[\mathrm{C} @ \mathrm{H}] 1 \mathrm{O}[\mathrm{C} @ @ \mathrm{H}] 1[\mathrm{C} @ @ \mathrm{H}](\backslash[ \\
\mathrm{NH}+]=\mathrm{C}(\mathrm{N}) / \mathrm{N})[\mathrm{C} @ \mathrm{H}](\mathrm{O})[\mathrm{C} @ @ \mathrm{H}](\mathrm{NH}+\mathrm{NH}=\mathrm{C}(\mathrm{N}) / \mathrm{N})[\mathrm{C} @ \mathrm{H}](\mathrm{O})[\mathrm{C} @ \mathrm{H}] \\
1 \mathrm{O}) \mathrm{C}\end{array}$ \\
\hline Penicillin G & $\begin{array}{l}\mathrm{S} 1[\mathrm{C} @ \mathrm{H}] 2 \mathrm{~N}([\mathrm{C} @ @ \mathrm{H}](\mathrm{C}(=\mathrm{O})[\mathrm{O}- \\
]) \mathrm{C} 1(\mathrm{C}) \mathrm{C}) \mathrm{C}(=\mathrm{O})[\mathrm{C} @ \mathrm{H}] 2 \mathrm{NC}(=\mathrm{O}) \mathrm{Cc} 1 \mathrm{ccccc} 1\end{array}$ \\
\hline Sperguallin & $\begin{array}{l}\mathrm{O}=\mathrm{C}(\mathrm{N}[\mathrm{C} @ @ \mathrm{H}](\mathrm{O}) \mathrm{C}(=\mathrm{O}) \mathrm{NCCCC}[\mathrm{NH} 2+] \mathrm{CCC}[\mathrm{NH} 3+]) \mathrm{C}[\mathrm{C} @ @ \mathrm{H}](\mathrm{O}) \mathrm{C} \\
\mathrm{CCC} \backslash[\mathrm{NH}+]=\mathrm{C}(\backslash \mathrm{N}) / \mathrm{N}\end{array}$ \\
\hline Rapamycin & $\begin{array}{l}\mathrm{O} 1[\mathrm{C} @ @ \mathrm{H}](\mathrm{CC}=\mathrm{O})[\mathrm{C} @ @ \mathrm{H}](\mathrm{C}=\mathrm{C}(/ \mathrm{C}) \backslash \mathrm{C} @ @ \mathrm{H}](\mathrm{O})[\mathrm{C} @ @ \mathrm{H}](\mathrm{OC}) \mathrm{C}(= \\
\mathrm{O})[\mathrm{C} @ @ \mathrm{H}](\mathrm{C}[\mathrm{C} @ @ \mathrm{H}](\mathrm{C}=\mathrm{C} \backslash \mathrm{C}=\mathrm{C} \backslash \mathrm{C}=\mathrm{C}(/ \mathrm{C}) \backslash \mathrm{C} @ @ \mathrm{H}](\mathrm{OC}) \mathrm{C}[\mathrm{C} @ \mathrm{H}] 2 \mathrm{O}[ \\
\mathrm{C} @](\mathrm{O})([\mathrm{C} @ @ \mathrm{H}](\mathrm{CC} 2) \mathrm{C}) \mathrm{C}(=\mathrm{O}) \mathrm{C}(=\mathrm{O}) \mathrm{N} 2[\mathrm{C} @ @ \mathrm{H}](\mathrm{CCCC}) \mathrm{C} 1=\mathrm{O}) \mathrm{C}) \mathrm{C} \\
) \mathrm{C})[\mathrm{C} @ @ \mathrm{H}](\mathrm{C}[\mathrm{C} @ \mathrm{H}] 1 \mathrm{C}[\mathrm{C} @ @ \mathrm{H}](\mathrm{OC})[\mathrm{C} @ \mathrm{H}](\mathrm{O}) \mathrm{CC} 1) \mathrm{C}\end{array}$ \\
\hline Echinocandin B & $\begin{array}{l}\mathrm{Oc1ccc}(\mathrm{cc} 1)[\mathrm{C} @ \mathrm{H}](\mathrm{O})[\mathrm{C} @ @ \mathrm{H}](\mathrm{O})[\mathrm{C} @ @ \mathrm{H}] 1 \mathrm{NC}(=\mathrm{O})[\mathrm{C} @ \mathrm{H}] 2 \mathrm{~N}(\mathrm{C}[\mathrm{C} @ \\
\mathrm{H}](\mathrm{O}) \mathrm{C} 2) \mathrm{C}(=\mathrm{O})[\mathrm{C} @ @ \mathrm{H}](\mathrm{NC}(=\mathrm{O})[\mathrm{C} @ @ \mathrm{H}](\mathrm{NC}=\mathrm{O}) \mathrm{CCCCCCC} \mathrm{C}=\mathrm{C} / \mathrm{C} \backslash \\
\mathrm{C}=\mathrm{C} / \mathrm{CCCCC}) \mathrm{C}[\mathrm{C} @ @ \mathrm{H}](\mathrm{O})[\mathrm{C} @ @ \mathrm{H}](\mathrm{O}) \mathrm{NC}(=\mathrm{O})[\mathrm{C} @ \mathrm{H}] 2 \mathrm{~N}(\mathrm{C}[\mathrm{C} @ \mathrm{H}](\mathrm{C}) \\
[\mathrm{C} @ \mathrm{H}] 2 \mathrm{O}) \mathrm{C}(=\mathrm{O})[\mathrm{C} @ @ \mathrm{H}](\mathrm{NC} 1=\mathrm{O})[\mathrm{C} @ \mathrm{H}](\mathrm{O}) \mathrm{C})[\mathrm{C} @ \mathrm{H}](\mathrm{O}) \mathrm{C}\end{array}$ \\
\hline Ginkgolide B & $\begin{array}{l}\mathrm{O} 1[\mathrm{C} @ @ \mathrm{H}] 2[\mathrm{C} @ @](\mathrm{O})([\mathrm{C} @] 34 \mathrm{O}[\mathrm{C} @ @ \mathrm{H}] 5 \mathrm{OC}(=\mathrm{O})[\mathrm{C} @ \mathrm{H}](\mathrm{O})[\mathrm{C} @] 56 \\
[\mathrm{C} @] 3([\mathrm{C} @ \mathrm{H}](\mathrm{OC} 4=\mathrm{O}) \mathrm{C}[\mathrm{C} @ \mathrm{H}] 6 \mathrm{C}(\mathrm{C})(\mathrm{C}) \mathrm{C})[\mathrm{C} @ \mathrm{H}] 2 \mathrm{O})[\mathrm{C} @ \mathrm{H}](\mathrm{C}) \mathrm{C} 1=\mathrm{O}\end{array}$ \\
\hline Vancomycin & $\begin{array}{l}\mathrm{Clc1} 2 \mathrm{Oc} 3 \mathrm{cc} 4[\mathrm{C} @ @ \mathrm{H}](\mathrm{NC}(=\mathrm{O})[\mathrm{C} @ @ \mathrm{H}](\mathrm{NC}(=\mathrm{O})[\mathrm{C} @ \mathrm{H}](\mathrm{NC}(=\mathrm{O})[\mathrm{C} @ \\
\mathrm{H}]([\mathrm{NH} 2+] \mathrm{C}) \mathrm{CC}(\mathrm{C}) \mathrm{C})[\mathrm{C} @ \mathrm{H}](\mathrm{O}) \mathrm{c}(\mathrm{c} 1) \mathrm{cc} 2) \mathrm{CC}(=\mathrm{O}) \mathrm{N}) \mathrm{C}(=\mathrm{O}) \mathrm{N}[\mathrm{C} @ @ \mathrm{H}] 1 \mathrm{c}\end{array}$ \\
\hline
\end{tabular}




\begin{tabular}{|c|c|}
\hline & $\begin{array}{l}\text { 2cc(- } \\
\mathrm{c5c}(\mathrm{cc}(\mathrm{O}) \mathrm{cc} 5 \mathrm{O})[\mathrm{C} @ \mathrm{H}](\mathrm{NC}(=\mathrm{O})[\mathrm{C} @ @ \mathrm{H}](\mathrm{NC} 1=\mathrm{O})[\mathrm{C} @ \mathrm{H}](\mathrm{O}) \mathrm{c} 1 \mathrm{cc}(\mathrm{Cl}) \mathrm{c}( \\
\mathrm{Oc}(\mathrm{c} 4) \mathrm{c} 3 \mathrm{O}[\mathrm{C} @ @ \mathrm{H}] 3 \mathrm{O}[\mathrm{C} @ \mathrm{H}](\mathrm{CO})[\mathrm{C} @ @ \mathrm{H}](\mathrm{O})[\mathrm{C} @ \mathrm{H}](\mathrm{O})[\mathrm{C} @ \mathrm{H}] 3 \mathrm{O}[\mathrm{C} \\
@ @ \mathrm{H}] 3 \mathrm{O}[\mathrm{C} @ @ \mathrm{H}](\mathrm{C})[\mathrm{C} @ @ \mathrm{H}](\mathrm{O})[\mathrm{C} @ @]([\mathrm{NH} 3+])(\mathrm{C} 3) \mathrm{C}) \mathrm{cc} 1) \mathrm{C}(=\mathrm{O})[\mathrm{O} \\
-]) \mathrm{c}(\mathrm{O}) \mathrm{cc} 2\end{array}$ \\
\hline Amphotericin B & $\begin{array}{l}\mathrm{O} 1[\mathrm{C} @ @ \mathrm{H}](\mathrm{C})[\mathrm{C} @ \mathrm{H}](\mathrm{C})[\mathrm{C} @ \mathrm{H}](\mathrm{O})[\mathrm{C} @ \mathrm{H}](\mathrm{C}=\mathrm{C} \backslash \mathrm{C}=\mathrm{C} \backslash \mathrm{C}=\mathrm{C} \backslash \mathrm{C}=\mathrm{C} \backslash \mathrm{C}=\mathrm{C} \backslash \\
\mathrm{C}=\mathrm{C} \backslash \mathrm{C}=\mathrm{C} \backslash[\mathrm{C} @ \mathrm{H}](\mathrm{O}[\mathrm{C} @ @ \mathrm{H}] 2 \mathrm{O}[\mathrm{C} @ \mathrm{H}](\mathrm{C})[\mathrm{C} @ @ \mathrm{H}](\mathrm{O})[\mathrm{C} @ \mathrm{H}]([\mathrm{NH} 3+]) \\
[\mathrm{C} @ @ \mathrm{H}] 2 \mathrm{O}) \mathrm{C}[\mathrm{C} @ @ \mathrm{H}] 2 \mathrm{O}[\mathrm{C} @ @](\mathrm{O})(\mathrm{C}[\mathrm{C} @ \mathrm{H}](\mathrm{O})[\mathrm{C} @ \mathrm{H}] 2 \mathrm{C}(=\mathrm{O})[\mathrm{O}- \\
]) \mathrm{C}[\mathrm{C} @ @ \mathrm{H}](\mathrm{O}) \mathrm{C}[\mathrm{C} @ @ \mathrm{H}](\mathrm{O})[\mathrm{C} @ \mathrm{H}](\mathrm{O}) \mathrm{CC}[\mathrm{C} @ @ \mathrm{H}](\mathrm{O}) \mathrm{C}[\mathrm{C} @ @ \mathrm{H}](\mathrm{O}) \\
\mathrm{CC} 1=\mathrm{O}) \mathrm{C}\end{array}$ \\
\hline Radicicol & $\begin{array}{l}\mathrm{Clc1c} 2 \mathrm{c}(\mathrm{C}(\mathrm{O}[\mathrm{C} @ @ \mathrm{H}](\mathrm{C}[\mathrm{C} @ \mathrm{H}] 3 \mathrm{O}[\mathrm{C} @ @ \mathrm{H}] 3 / \mathrm{C}=\mathrm{ClC}=\mathrm{C} \backslash \mathrm{C}(=\mathrm{O}) \mathrm{C} 2) \mathrm{C})=\mathrm{O} \\
) \mathrm{c}(\mathrm{O}) \mathrm{cc} 1 \mathrm{O}\end{array}$ \\
\hline $\begin{array}{l}\text { Salicylihalamide } \\
\text { A }\end{array}$ & $\begin{array}{l}\mathrm{O} 1[\mathrm{C} @ \mathrm{H}](\mathrm{C}[\mathrm{C} @ @ \mathrm{H}](\mathrm{O})[\mathrm{C} @ \mathrm{H}](\mathrm{C} \backslash \mathrm{C}=\mathrm{C} \backslash \mathrm{Cc} 2 \mathrm{c}(\mathrm{C} 1=\mathrm{O}) \mathrm{c}(\mathrm{O}) \mathrm{ccc} 2) \mathrm{C}) \mathrm{C} \backslash \mathrm{C}=\mathrm{C} \\
\backslash \mathrm{NC}(=\mathrm{O}) / \mathrm{C}=\mathrm{C} \backslash \mathrm{C}=\mathrm{C} / \mathrm{CC}\end{array}$ \\
\hline Telomestatin & $\begin{array}{l}\mathrm{S} 1 \mathrm{C} 2=\mathrm{N}[\mathrm{C} @ \mathrm{H}](\mathrm{c} 3 \mathrm{oc}(\mathrm{c}(\mathrm{n} 3)-\mathrm{c} 3 \mathrm{oc}(\mathrm{c}(\mathrm{n} 3)-\mathrm{c} 3 \mathrm{occ}(\mathrm{n} 3)-\mathrm{c} 3 \mathrm{occ}(\mathrm{n} 3)-\mathrm{c} 3 \mathrm{occ}(\mathrm{n} 3)- \\
\mathrm{c} 3 \mathrm{occ}(\mathrm{n} 3)-\mathrm{c} 3 \mathrm{occ} 2 \mathrm{n} 3) \mathrm{C}) \mathrm{C}) \mathrm{C} 1\end{array}$ \\
\hline Rifamycin B & $\begin{array}{l}\mathrm{O} 1 \mathrm{c} 2 \mathrm{c} 3 \mathrm{c} 4 \mathrm{c}(\mathrm{c}(\mathrm{O}) \mathrm{c} 2 \mathrm{C}) \mathrm{c}(\mathrm{O}) \mathrm{c}(\mathrm{NC}(=\mathrm{O}) / \mathrm{C}(=\mathrm{C} \backslash \mathrm{C}=\mathrm{C} \backslash \mathrm{C} @ \mathrm{H}](\mathrm{C})[\mathrm{C} @ \mathrm{H}](\mathrm{O})[\mathrm{C} \\
@ @ \mathrm{H}](\mathrm{C})[\mathrm{C} @ @ \mathrm{H}](\mathrm{O})[\mathrm{C} @ @ \mathrm{H}](\mathrm{C})[\mathrm{C} @ \mathrm{H}](\mathrm{OC}(=\mathrm{O}) \mathrm{C})[\mathrm{C} @ \mathrm{H}](\mathrm{C})[\mathrm{C} @ @ \\
\mathrm{H}](\mathrm{OC}) \backslash \mathrm{C}=\mathrm{C} \backslash \mathrm{O}[\mathrm{C} @] 1(\mathrm{C}) \mathrm{C} 3=\mathrm{O}) / \mathrm{C}) \mathrm{cc} 4 \mathrm{OCC}(=\mathrm{O})[\mathrm{O}-]\end{array}$ \\
\hline Apoptolidin & $\begin{array}{l}\mathrm{O} 1[\mathrm{C} @ @ \mathrm{H}](\mathrm{C}[\mathrm{C} @ \mathrm{H}](\mathrm{OC})[\mathrm{C} @ @ \mathrm{H}](\mathrm{O}) \mathrm{CC} \backslash \mathrm{C}=\mathrm{C}(\mathrm{C}=\mathrm{C} \backslash \mathrm{C} @ \mathrm{H}](\mathrm{O}[\mathrm{C} @ @ \\
\mathrm{H}] 2 \mathrm{O}[\mathrm{C} @ @ \mathrm{H}](\mathrm{C})[\mathrm{C} @ \mathrm{H}](\mathrm{OC})[\mathrm{C} @ @ \mathrm{H}](\mathrm{O})[\mathrm{C} @ @ \mathrm{H}] 2 \mathrm{O})[\mathrm{C} @ @ \mathrm{H}](\mathrm{C} \backslash \mathrm{C}= \\
\mathrm{C}(\mathrm{C}=\mathrm{C}(\backslash \mathrm{C}=\mathrm{C}(/ \mathrm{C}) \backslash \mathrm{C} 1=\mathrm{O}) / \mathrm{C}) / \mathrm{C}) \mathrm{C}) / \mathrm{C})[\mathrm{C} @ @ \mathrm{H}](\mathrm{O})[\mathrm{C} @ @] 1(\mathrm{O}[\mathrm{C} @ \mathrm{H}](\mathrm{C}[ \\
\mathrm{C} @ @ \mathrm{H}](\mathrm{O}[\mathrm{C} @ @ \mathrm{H}] 2 \mathrm{O}[\mathrm{C} @ @ \mathrm{H}](\mathrm{C})[\mathrm{C} @ \mathrm{H}](\mathrm{O}[\mathrm{C} @ @ \mathrm{H}] 3 \mathrm{O}[\mathrm{C} @ \mathrm{H}](\mathrm{C})[\mathrm{C} \\
@ @ \mathrm{H}](\mathrm{O})[\mathrm{C} @ \mathrm{H}](\mathrm{OC}) \mathrm{C} 3)[\mathrm{C} @ @](\mathrm{O})(\mathrm{C} 2) \mathrm{C}) \mathrm{COC})[\mathrm{C} @ \mathrm{H}](\mathrm{C})[\mathrm{C} @ \mathrm{H}](\mathrm{O})[ \\
\mathrm{C} @ \mathrm{H}] 1 \mathrm{C}) \mathrm{O}\end{array}$ \\
\hline $\begin{array}{l}\text { Midecamycin } \\
\text { A1 }\end{array}$ & $\begin{array}{l}\mathrm{O} 1[\mathrm{C} @ @ \mathrm{H}](\mathrm{ClC}=\mathrm{ClC}=\mathrm{C} \backslash \mathrm{C} @ \mathrm{H}](\mathrm{O})[\mathrm{C} @ @ \mathrm{H}](\mathrm{C}[\mathrm{C} @ \mathrm{H}](\mathrm{CC}=\mathrm{O})[\mathrm{C} @ \mathrm{H}]( \\
\mathrm{O}[\mathrm{C} @ @ \mathrm{H}] 2 \mathrm{O}[\mathrm{C} @ \mathrm{H}](\mathrm{C})[\mathrm{C} @ @ \mathrm{H}](\mathrm{O}[\mathrm{C} @ @ \mathrm{H}] 3 \mathrm{O}[\mathrm{C} @ @ \mathrm{H}](\mathrm{C})[\mathrm{C} @ \mathrm{H}](\mathrm{O} \\
\mathrm{C}(=\mathrm{O}) \mathrm{CC})[\mathrm{C} @](\mathrm{O})(\mathrm{C} 3) \mathrm{C})[\mathrm{C} @ \mathrm{H}]([\mathrm{NH}+](\mathrm{C}) \mathrm{C})[\mathrm{C} @ \mathrm{H}] 2 \mathrm{O})[\mathrm{C} @ @ \mathrm{H}](\mathrm{OC})[ \\
\mathrm{C} @ \mathrm{H}](\mathrm{OC}(=\mathrm{O}) \mathrm{CC}) \mathrm{CC}=\mathrm{O}) \mathrm{C}) \mathrm{C}\end{array}$ \\
\hline Zaragozic acid & $\begin{array}{l}\mathrm{O} 1[\mathrm{C} @ @] 2(\mathrm{C}(=\mathrm{O})[\mathrm{O}- \\
])[\mathrm{C} @](\mathrm{O})(\mathrm{C}(\mathrm{O})=\mathrm{O})[\mathrm{C} @ \mathrm{H}](\mathrm{O}[\mathrm{C} @] 1(\mathrm{CCC}([\mathrm{C} @ @ \mathrm{H}](\mathrm{OC}(=\mathrm{O}) \mathrm{C})[\mathrm{C} @ @ \mathrm{H}\end{array}$ \\
\hline
\end{tabular}




\begin{tabular}{|c|c|}
\hline $\mathbf{A}$ & $\begin{array}{l}](\mathrm{Cc} 1 \operatorname{ccccc} 1) \mathrm{C})=\mathrm{C})[\mathrm{C} @ \mathrm{H}](\mathrm{O})[\mathrm{C} @ \mathrm{H}] 2 \mathrm{OC}(=\mathrm{O}) \backslash \mathrm{C}=\mathrm{C} \backslash[\mathrm{C} @ \mathrm{H}](\mathrm{C}[\mathrm{C} @ \mathrm{H}](\mathrm{C} \\
\mathrm{C}) \mathrm{C}) \mathrm{C}) \mathrm{C}(=\mathrm{O})[\mathrm{O}-]\end{array}$ \\
\hline Talaromycin B & O1C[C@H](CO)[C@@H](O)C[C@]12OC[C@@H](CC2)CC \\
\hline Spongistatin 1 & $\begin{array}{l}\mathrm{ClC}(\mathrm{C}=\mathrm{C} \backslash \mathrm{C} @ @ \mathrm{H}](\mathrm{O}) \mathrm{CC}(\mathrm{C}[\mathrm{C} @ \mathrm{H}] 1 \mathrm{O}[\mathrm{C} @ \mathrm{H}] 2[\mathrm{C} @ \mathrm{H}](\mathrm{O})[\mathrm{C} @ @] 3(\mathrm{O}[\mathrm{C} \\
@ \mathrm{H}](\mathrm{CCCl}=\mathrm{C} /[\mathrm{C} @ @ \mathrm{H}] 4 \mathrm{O}[\mathrm{C} @ @] 5(\mathrm{O}[\mathrm{C} @ \mathrm{H}](\mathrm{CC}=\mathrm{O})[\mathrm{C} @ @ \mathrm{H}](\mathrm{C})[\mathrm{C} \\
@ \mathrm{H}](\mathrm{OC}(=\mathrm{O}) \mathrm{C})[\mathrm{C} @ \mathrm{H}](\mathrm{C}) \mathrm{C}(\mathrm{C}[\mathrm{C} @ @ \mathrm{H}] 6 \mathrm{O}[\mathrm{C} @ @] 7(\mathrm{O}[\mathrm{C} @ \mathrm{H}](\mathrm{CC}( \\
\mathrm{O}[\mathrm{C} @ @ \mathrm{H}]([\mathrm{C} @ @ \mathrm{H}] 1 \mathrm{O})[\mathrm{C} @ \mathrm{H}] 2 \mathrm{C})=\mathrm{O}) \mathrm{C}[\mathrm{C} @ \mathrm{H}](\mathrm{OC}(=\mathrm{O}) \mathrm{C}) \mathrm{C} 7) \mathrm{C}[\mathrm{C} @ @ \\
](\mathrm{O})(\mathrm{C} 6) \mathrm{C})=\mathrm{C}) \mathrm{C}[\mathrm{C} @ \mathrm{H}](\mathrm{OC}) \mathrm{C} 5) \mathrm{C}[\mathrm{C} @ @ \mathrm{H}](\mathrm{O}) \mathrm{C} 4)[\mathrm{C} @ \mathrm{H}](\mathrm{C})[\mathrm{C} @ @ \mathrm{H}]( \\
\mathrm{O}) \mathrm{C} 3) \mathrm{O})=\mathrm{C})=\mathrm{C}\end{array}$ \\
\hline Brevetoxin B & 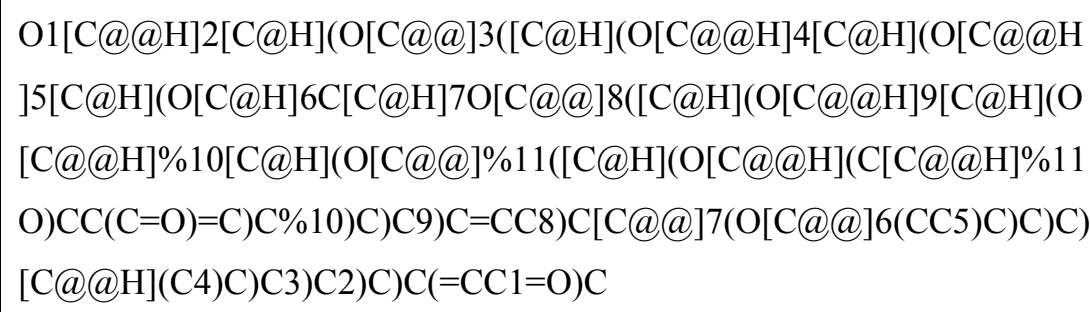 \\
\hline Quinine & $\begin{array}{l}\mathrm{O}(\mathrm{C}) \mathrm{c} 1 \mathrm{cc} 2 \mathrm{c}(\mathrm{nccc} 2[\mathrm{C} @ @ \mathrm{H}](\mathrm{O})[\mathrm{C} @ \mathrm{H}] 2[\mathrm{~N} @ @ \mathrm{H}+] 3 \mathrm{C}[\mathrm{C} @ @ H]([\mathrm{C} @ \mathrm{H}]( \\
\mathrm{C} 2) \mathrm{CC} 3) \mathrm{C}=\mathrm{C}) \mathrm{cc} 1\end{array}$ \\
\hline Mycobactin S & $\begin{array}{l}\mathrm{O} 1 \mathrm{C}[\mathrm{C} @ \mathrm{H}](\mathrm{N}=\mathrm{C} 1 \mathrm{cl} \operatorname{ccccc} 1 \mathrm{O}) \mathrm{C}(=\mathrm{O}) \mathrm{N}[\mathrm{C} @ @ \mathrm{H}](\mathrm{CCCCN}([\mathrm{O}- \\
]) \mathrm{C}(=\mathrm{O}) \backslash \mathrm{C}=\mathrm{C} / \mathrm{CCCCCCCCCCCCC}) \mathrm{C}(\mathrm{O}[\mathrm{C} @ \mathrm{H}](\mathrm{CC}(=\mathrm{O}) \mathrm{N}[\mathrm{C} @ \mathrm{H}] 1 \mathrm{CC} \\
\mathrm{CCN}([\mathrm{O}-]) \mathrm{C} 1=\mathrm{O}) \mathrm{C})=\mathrm{O}\end{array}$ \\
\hline Duocarmycin $\mathbf{A}$ & $\begin{array}{l}\mathrm{O}(\mathrm{C}) \mathrm{c} 1 \mathrm{c}(\mathrm{OC}) \mathrm{c} 2[\mathrm{nH}] \mathrm{c}(\mathrm{cc} 2 \mathrm{cc} 1 \mathrm{OC}) \mathrm{C}(=\mathrm{O}) \mathrm{N} 1 \mathrm{C}=2[\mathrm{C} @] 3([\mathrm{C} @ \mathrm{H}](\mathrm{C} 3) \mathrm{C} 1) \mathrm{C} 1 \\
=\mathrm{C}(\mathrm{N}[\mathrm{C} @](\mathrm{C}(\mathrm{OC})=\mathrm{O})(\mathrm{C}) \mathrm{C} 1=\mathrm{O}) \mathrm{C}(=\mathrm{O}) \mathrm{C}=2\end{array}$ \\
\hline Bleomycin A2 & 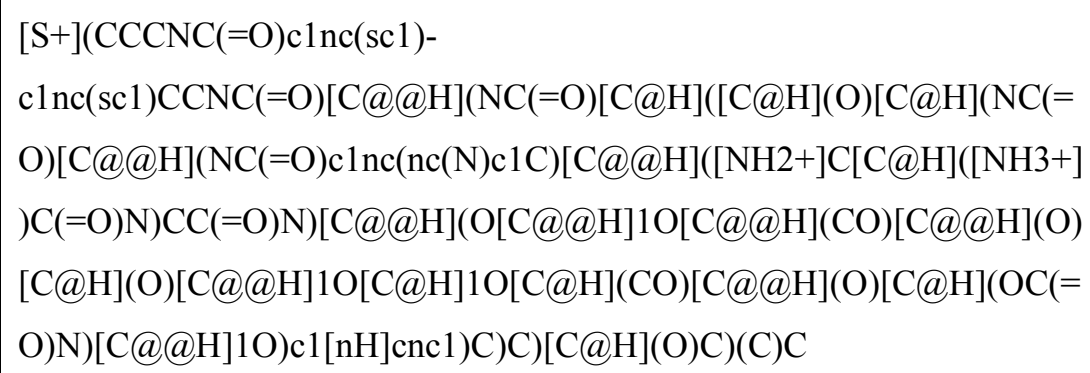 \\
\hline Brefeldin A & $\begin{array}{l}\mathrm{O} 1[\mathrm{C} @ \mathrm{H}](\mathrm{CCC} \backslash \mathrm{C}=\mathrm{C} \backslash[\mathrm{C} @ \mathrm{H}] 2[\mathrm{C} @ @ H](\mathrm{C}[\mathrm{C} @ @ H](\mathrm{O}) \mathrm{C} 2)[\mathrm{C} @ \mathrm{H}](\mathrm{O}) \backslash \mathrm{C} \\
=\mathrm{ClC} 1=\mathrm{O}) \mathrm{C}\end{array}$ \\
\hline Cytochalasin B & O1[C@@]23[C@@H]([C@H](C)C(=C)[C@@H](O)[C@@H]2lC=ClC \\
\hline
\end{tabular}




\begin{tabular}{|c|c|}
\hline & $\mathrm{C} @ @ \mathrm{H}](\mathrm{CCC}[\mathrm{C} @ @ \mathrm{H}](\mathrm{O}) \backslash \mathrm{C}=\mathrm{C} \backslash \mathrm{C} 1=\mathrm{O}) \mathrm{C})[\mathrm{C} @ @ \mathrm{H}](\mathrm{NC} 3=\mathrm{O}) \mathrm{Cc} 1 \mathrm{cccc} 1$ \\
\hline Epothilone A & $\begin{array}{l}\mathrm{s} 1 \mathrm{cc}(\mathrm{nc} 1 \mathrm{C}) \backslash \mathrm{C}=\mathrm{C}(/ \mathrm{C}) \backslash \mathrm{C} @ \mathrm{H}] 1 \mathrm{OC}(=\mathrm{O}) \mathrm{C}[\mathrm{C} @ \mathrm{H}](\mathrm{O}) \mathrm{C}(\mathrm{C})(\mathrm{C}) \mathrm{C}(=\mathrm{O})[\mathrm{C} @ \mathrm{H}]( \\
\mathrm{C})[\mathrm{C} @ @ \mathrm{H}](\mathrm{O})[\mathrm{C} @ \mathrm{H}](\mathrm{CCC}[\mathrm{C} @ \mathrm{H}] 2 \mathrm{O}[\mathrm{C} @ \mathrm{H}] 2 \mathrm{C} 1) \mathrm{C}\end{array}$ \\
\hline Lactacystin & $\begin{array}{l}\mathrm{S}(\mathrm{C}=\mathrm{O})[\mathrm{C} @ @] 1(\mathrm{NC}(=\mathrm{O})[\mathrm{C} @ \mathrm{H}](\mathrm{C})[\mathrm{C} @ @ \mathrm{H}] 1 \mathrm{O})[\mathrm{C} @ @ \mathrm{H}](\mathrm{O}) \mathrm{C}(\mathrm{C}) \mathrm{C}) \mathrm{C}[ \\
\mathrm{C} @ \mathrm{H}](\mathrm{NC}(=\mathrm{O}) \mathrm{C}) \mathrm{C}(=\mathrm{O})[\mathrm{O}-]\end{array}$ \\
\hline $\begin{array}{l}\text { Calicheamicin } \\
\gamma 1\end{array}$ & 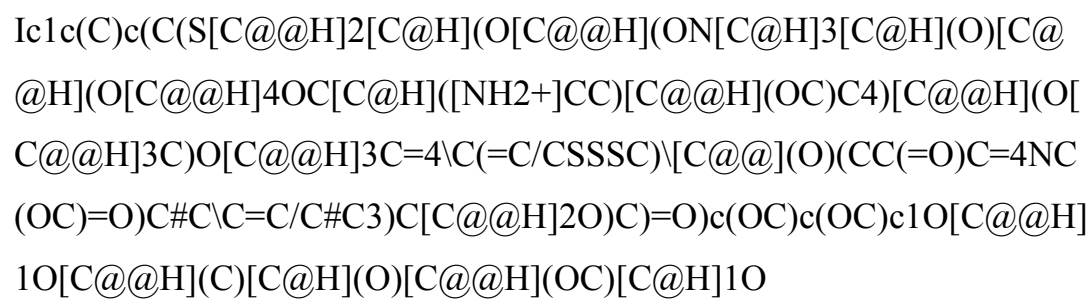 \\
\hline Artemisinin & $\begin{array}{l}\mathrm{O} 1[\mathrm{C} @ @ \mathrm{H}] 2 \mathrm{O}[\mathrm{C} @ @] 3(\mathrm{OO}[\mathrm{C} @] 24[\mathrm{C} @ @ H](\mathrm{CC}[\mathrm{C} @ \mathrm{H}]([\mathrm{C} @ @ \mathrm{H}] 4 \mathrm{CC} \\
3) \mathrm{C})[\mathrm{C} @ @ \mathrm{H}](\mathrm{C}) \mathrm{C} 1=\mathrm{O}) \mathrm{C}\end{array}$ \\
\hline Compactin & $\begin{array}{l}\mathrm{O} 1[\mathrm{C} @ @ \mathrm{H}](\mathrm{C}[\mathrm{C} @ @ \mathrm{H}](\mathrm{O}) \mathrm{CC} 1=\mathrm{O}) \mathrm{CC}[\mathrm{C} @ @ \mathrm{H}] 1[\mathrm{C} @ @ \mathrm{H}] 2 \mathrm{C}(\mathrm{C}=\mathrm{C}[\mathrm{C} @ \text {, } \\
@ \mathrm{H}] 1 \mathrm{C})=\mathrm{CCC}[\mathrm{C} @ @ \mathrm{H}] 2 \mathrm{OC}(=\mathrm{O})[\mathrm{C} @ \mathrm{H}](\mathrm{CC}) \mathrm{C}\end{array}$ \\
\hline Lipstatin & $\begin{array}{l}\mathrm{O} 1[\mathrm{C} @ @ \mathrm{H}](\mathrm{C}[\mathrm{C} @ @ \mathrm{H}](\mathrm{OC}(=\mathrm{O})[\mathrm{C} @ @ \mathrm{H}](\mathrm{NC}=\mathrm{O}) \mathrm{CC}(\mathrm{C}) \mathrm{C}) \mathrm{C} \backslash \mathrm{C}=\mathrm{C} / \mathrm{C} \backslash \mathrm{C}= \\
\mathrm{C} / \mathrm{CCCCC})[\mathrm{C} @ \mathrm{H}](\mathrm{CCCCC}) \mathrm{C} 1=\mathrm{O}\end{array}$ \\
\hline $\begin{array}{l}\text { Pseudomonic } \\
\text { acid A }\end{array}$ & $\begin{array}{l}\mathrm{O} 1[\mathrm{C} @ @ \mathrm{H}](\mathrm{C}[\mathrm{C} @ \mathrm{H}] 2 \mathrm{CO}[\mathrm{C} @ @ \mathrm{H}](\mathrm{ClC}=\mathrm{ClC}(\mathrm{OCCCCCCCC}(=\mathrm{O})[\mathrm{O}- \\
])=\mathrm{O}) \backslash \mathrm{C})[\mathrm{C} @ \mathrm{H}](\mathrm{O})[\mathrm{C} @ @ \mathrm{H}] 2 \mathrm{O})[\mathrm{C} @ @ \mathrm{H}] 1[\mathrm{C} @ \mathrm{H}]([\mathrm{C} @ @ \mathrm{H}](\mathrm{O}) \mathrm{C}) \mathrm{C}\end{array}$ \\
\hline Daptomycin & $\begin{array}{l}\mathrm{O} 1[\mathrm{C} @ \mathrm{H}](\mathrm{C})[\mathrm{C} @ \mathrm{H}](\mathrm{NC}(=\mathrm{O})[\mathrm{C} @ @ \mathrm{H}](\mathrm{NC}(=\mathrm{O})[\mathrm{C} @ \mathrm{H}](\mathrm{NC}(=\mathrm{O})[\mathrm{C} @ @ \\
\mathrm{H}](\mathrm{NC}(=\mathrm{O}) \mathrm{CCCCCCCCC}) \mathrm{Cc} 2 \mathrm{c} 3 \mathrm{c}([\mathrm{nH}] \mathrm{c} 2) \mathrm{ccc} 3) \mathrm{CC}(=\mathrm{O}) \mathrm{N}) \mathrm{CC}(=\mathrm{O})[\mathrm{O}- \\
]) \mathrm{C}(=\mathrm{O}) \mathrm{NCC}(=\mathrm{O}) \mathrm{N}[\mathrm{C} @ @ \mathrm{H}](\mathrm{CCC}[\mathrm{NH} 3+]) \mathrm{C}(=\mathrm{O}) \mathrm{N}[\mathrm{C} @ @ \mathrm{H}](\mathrm{CC}(=\mathrm{O})[\mathrm{O} \\
-]) \mathrm{C}(=\mathrm{O}) \mathrm{N}[\mathrm{C} @ \mathrm{H}](\mathrm{C}) \mathrm{C}(=\mathrm{O}) \mathrm{N}[\mathrm{C} @ @ \mathrm{H}](\mathrm{CC}(=\mathrm{O})[\mathrm{O}- \\
]) \mathrm{C}(=\mathrm{O}) \mathrm{NCC}(=\mathrm{O}) \mathrm{N}[\mathrm{C} @ \mathrm{H}](\mathrm{CO}) \mathrm{C}(=\mathrm{O}) \mathrm{N}[\mathrm{C} @ @ \mathrm{H}]([\mathrm{C} @ @ \mathrm{H}](\mathrm{CC}=\mathrm{O})[\mathrm{O}- \\
]) \mathrm{C}) \mathrm{C}(=\mathrm{O}) \mathrm{N}[\mathrm{C} @ @ \mathrm{H}](\mathrm{CC}(=\mathrm{O}) \mathrm{c} 2 \mathrm{cccc} 2 \mathrm{~N}) \mathrm{C} 1=\mathrm{O}\end{array}$ \\
\hline Bestatin & $\begin{array}{l}\mathrm{O}=\mathrm{C}(\mathrm{N}[\mathrm{C} @ @ \mathrm{H}](\mathrm{CC}(\mathrm{C}) \mathrm{C}) \mathrm{C}(=\mathrm{O})[\mathrm{O}- \\
])[\mathrm{C} @ @ \mathrm{H}](\mathrm{O})[\mathrm{C} @ \mathrm{H}]([\mathrm{NH} 3+]) \mathrm{Cc} 1 \mathrm{ccccc} 1\end{array}$ \\
\hline Plaunotol & $\mathrm{OC} / \mathrm{C}(=\mathrm{C} \backslash \mathrm{CC} \backslash \mathrm{C}(=\mathrm{C} \backslash \mathrm{CO}) \backslash \mathrm{C}) / \mathrm{CC} \backslash \mathrm{C}=\mathrm{C}(\backslash \mathrm{CC} \backslash \mathrm{C}=\mathrm{C}(\backslash \mathrm{C}) / \mathrm{C}) / \mathrm{C}$ \\
\hline Geldanamycin & $\mathrm{O}(\mathrm{C}) \mathrm{C} 1=\mathrm{C} 2 \mathrm{C}[\mathrm{C} @ \mathrm{H}](\mathrm{C}[\mathrm{C} @ \mathrm{H}](\mathrm{OC})[\mathrm{C} @ \mathrm{H}](\mathrm{O})[\mathrm{C} @ \mathrm{H}](\mathrm{C}=\mathrm{C}(/ \mathrm{C}) \backslash \mathrm{C} @ \mathrm{H}]($ \\
\hline
\end{tabular}




\section{${ }^{1} \mathrm{H},{ }^{13} \mathrm{C}$ and ${ }^{19} \mathrm{~F}$ Spectra of all new compounds}

It should be noted that most products are tert-butyl carbamates and, as such, they show broad signals both in the ${ }^{1} \mathrm{H}$ and the ${ }^{13} \mathrm{C}$ NMR spectra due to the presence of rotamers. In addition, some signals in the ${ }^{13} \mathrm{C}$ NMR spectra appear duplicated. In order to showcase that the presence of rotamers is responsible of the bad quality of these spectra two of them have been recorded at high temperature $\left(100^{\circ} \mathrm{C}\right)$ giving rise to single sets of signals. Compound 14b also exhibits rotamers due to lack of free rotation along the $\mathrm{C}-\mathrm{C}$ bond between the $\mathrm{C} \alpha$ of the enone and the 2-methoxyphenyl ring. 